PFC/JA-90-9

\author{
INFLUENCE OF TRAPPED-ELECTRON DISTRIBUTION ON THE \\ SIDEBAND INSTABILITY IN A \\ HELICAL WIGGLER FREE ELECTRON LASER \\ T.-Y. Brian Yang \\ Ronald C. Davidson \\ March, 1990
}

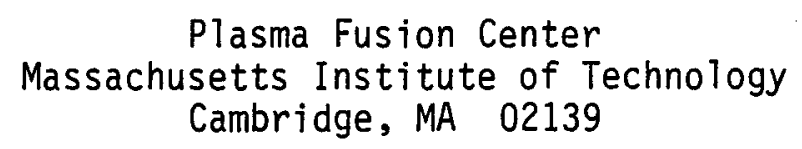

Research was supported in part by the National Science Foundation, the Department of Energy High Energy Physics Division and the Naval Research Laboratory Plasma Physics Division. 


\title{
Influence of Trapped-Electron Distribution on the Sideband Instability in a Helical Wiggler Free Electron Laser
}

\author{
T.-Y. Brian Yang and Ronald C. Davidson \\ Plasma Fusion Center \\ Massachusetts Institute of Technology \\ Cambridge, Massachusetts 02139
}

\begin{abstract}
Use is made of the Vlasov-Maxwell equations to investigate detailed properties of the sideband instability for a helical wiggler free-electron laser with wiggler wavelength $\lambda_{w}=2 \pi / k_{0}=$ const and normalized wiggler amplitude $a_{w}=e \hat{B}_{w} / m c^{2} k_{0}=$ const. The model describes the nonlinear evolution of a right-circularly polarized primary electromagnetic wave with frequency $\omega_{s}$, wavenumber $k_{s}$, and slowly varying amplitude $\hat{a}_{s}(z, t)$ and phase $\delta_{s}(z, t)$ (eikonal approximation). The coupled Vlasov and field-evolution equations are analyzed in the ponderomotive frame ("primed" variables) moving with velocity $v_{p}=\omega_{s} /\left(k_{s}+k_{0}\right)$ relative to the laboratory. Detailed properties of the sideband instability are investigated for small-amplitude perturbations about a quasi-steady state characterized by an equilibrium electron distribution $f^{0}\left(\gamma_{0}^{\prime}\right)$ and a primary electromagnetic wave with constant amplitude $\hat{a}_{s}^{0}=$ const (independent of $z^{\prime}$ and $t^{\prime}$ ) and slowly varying phase $\delta_{s}^{0}\left(z^{\prime}\right)$. A formal dispersion relation is derived for perturbations about a general equilibrium distribution $f^{0}\left(\gamma_{0}^{\prime}\right)$ which may include both trapped and untrapped electrons. For the case where only trapped electrons are present, the dispersion relation is reduced to a simple analytical form. Detailed properties of the sideband instability are investigated for the case where the trapped electrons uniformly populate the ponderomotive potential up to an energy $\gamma_{M}^{\prime} \leq \hat{\gamma}_{+}^{\prime}$, where $\hat{\gamma}_{+}^{\prime}$ is the energy at the separatrix. Analysis of the dispersion relation shows that the maximum energy of the trapped-electron population $\left(\gamma_{M}^{\prime}\right)$ significantly affects detailed stability properties in the strong-pump and intermediate-pump regimes.
\end{abstract}




\section{Introduction}

There is growing experimental ${ }^{1-20}$ and theoretical ${ }^{21-73}$ evidence that free-electron lasers (FEL's) ${ }^{74-80}$. are effective sources of coherent radiation generation by intense relativistic electron beams. Recent theoretical studies have included investigations of nonlinear effects ${ }^{21-49}$ and saturation mechanisms, the influence of finite radial geometry on linear stability properties, ${ }^{50-55}$ novel magnetic field geometries for radiation generation, ${ }^{50,56-60}$ and fundamental studies of stability behavior. ${ }^{61-72}$ One topic of considerable practical importance is the sideband instability ${ }^{5-8,21-28,39-49}$ which results from the bounce motion of electrons trapped in the (finite-amplitude) ponderomotive potential. It was first predicted theoretically by Kroll, Morton and Rosenbluth ${ }^{39}$ that the bounce motion of the trapped electrons can lead to the unstable development of sideband signals. Numerical simulations ${ }^{40-48}$ and experimental observations $^{5-8}$ subsequently demonstrated that sideband signals can grow to a significant level. In analytical investigations of the sideband instability, both kinetic ${ }^{21-23}$ and single-particle ${ }^{24,25,39}$ models have been developed. Making use of the Vlasov-Maxwell equations in the ponderomotive frame, Davidson ${ }^{21}$ investigated the sideband instability for perturbations about a self-consistent equilibrium state. In circumstances where the trapped electrons are localized near the bottom of the ponderomotive potential, it was found that the detailed stability properties are relatively insensitive to the form of the distribution of trapped electrons. In a subsequent analysis, ${ }^{22}$ the detailed dependence of the sideband instability on the system parameters was examined. Moreover, Davidson and Wurtele ${ }^{24}$ have developed a single-particle model (with appropriate statistical averages) to analyze the sideband instability in the ponderu-

motive frame. The effects of the untrapped electrons have also been considered. ${ }^{25}$ Most previous studies of the sideband instability are restricted to the case where the trapped electrons are "deeply" trapped in the ponderomotive potential. Recently, 
however, Riyopoulos and Tang ${ }^{26}$ developed a kinetic model to study the sideband instability for general distribution of trapped electrons. The influence of the sideband signal on the electron dynamics has also been investigated. ${ }^{27}$ Furthermore, Sharp and $\mathrm{Y}^{\prime} \mathrm{u}^{28}$ have developed a kinetic model of the sideband instability in which the transverse variations of the wave fields are calculated self-consistently from Maxwell's equations.

In this paper, we present a one-dimensional kinetic analysis of the sideband instability. Similar to Sharp and Yu's work, ${ }^{28}$ we consider general trapped-electron distribution and determine the self-consistent evolution of the electromagnetic fields. The present analysis differs from Sharp and Yu's work ${ }^{28}$ in the following important aspects:

(a) For general trapped-electron distribution $f^{0}\left(\gamma_{0}^{\prime}\right)$, the kinetic dispersion relation is reduced to a simple form [Eq.(130)] which involves integrals over only the energy variable $\gamma_{0}^{\prime}$.

(b) When the electrons uniformly populate the ponderomotive potential up to an energy level $\gamma_{M I}^{\prime}<\hat{\gamma}_{+}^{\prime}[$ Eq.(132)], the dispersion relation is obtained in closed analytical form [Eq.(141)].

(c) In the limiting case where the electrons uniformly populate the ponderomotive potential up to the separatrix $\left(\gamma_{M}^{\prime}=\hat{\gamma}_{+}^{\prime}\right)$, the dispersion relation is also obtained in closed analytical form [Eq.(103)].

(d) The present analysis is carried out in the ponderomotive frame, which leads to considerable simplification in the orbit equations [Eqs. (83) and (111)] and in the detailed investigation of the sideband instability. ${ }^{21-23,73,74}$

The theoretical model (Sec.II) employed in the present analysis is based on Vlasov: Maxwell equations. The basic equations and assumptions are described in Sec.II A. A tenuous, relativistic electron beam propagates through a constant-amplitude 
helical wiggler magnetic field with wavelength $\lambda_{w}=2 \pi / k_{0}=$ const and normalized amplitude $a_{w}=e \hat{B}_{w} / m c^{2} k_{0}$ [Eq.(1)]. The model neglects longitudinal perturbations (Compton-regime approximation with $\delta \phi \simeq 0$ ) and transverse spatial variations $(\partial / \partial x=0=\partial / \partial y)$. Moreover, the analysis is carried out for the case of a finite-amplitude primary electromagnetic wave $\left(\omega_{s}, k_{s}\right)$ with right-circular polarization and slowly varying normalized amplitude $\hat{a}_{s}(z, t)$ and wave phase $\delta_{s}(z, t)$ in the eikonal approximation [Eq.(2)]. In the ponderomotive frame [moving with velocity $v_{p}=\omega_{s} /\left(k_{s}+k_{0}\right)$ relative to the laboratory frame], the nonlinear evolution of the complex amplitude $a_{s}\left(z^{\prime}, t^{\prime}\right)=\hat{a}_{s}\left(z^{\prime}, t^{\prime}\right) \exp \left[i \delta_{0}^{\prime}\left(z^{\prime}, t^{\prime}\right)\right]$ is described by Eq.(19). It is also assumed in the present analysis that all electrons move on surfaces with zero transverse canonical momentum, with corresponding electron distribution function of the form $f_{b}\left(z^{\prime}, \mathbf{p}^{\prime}, t^{\prime}\right)=\delta\left(P_{x}^{\prime}\right) \delta\left(P_{y}^{\prime}\right) f\left(z^{\prime}, p_{z}^{\prime}, t^{\prime}\right)[$ Eq. (10)] in the ponderomotive frame. In Sec.II B, equilibrium properties are investigated. Under quasi-steady-state conditions $\left(\partial / \partial t^{\prime}=0\right)$ corresponding to the saturated state of a free-electron laser, the electron distribution function depends only on the energy constant of the motion $\gamma_{0}^{\prime}$ [Eq.(29)], and the primary electromagnetic wave has a constant amplitude $\hat{a}_{\text {g }}^{0}$ and a slowly spatially varying phase $\delta_{s}^{0}\left(z^{\prime}\right)[$ Eq.(28)]. In Sec.III, the linear stability analysis is presented. The method of characteristics is used to derive the kinetic dispersion relation [Eqs.(60) and (64)] from the linearized Vlasov-Maxwell equations (36) and $(39)$.

In Sec.IV, we apply the equilibrium and stability formalism developed in Secs. II and III to a particular choice of electron distribution function [Eq.(65)] in which the electrons uniformly populate the ponderomotive potential up to the separatrix $\left(\gamma_{0}^{\prime}=\hat{\gamma}_{+}^{\prime}\right)$. The dispersion relation for this particular distribution is obtained in closed analytical form [Eq.(103)]. In the limiting case where the dimensionless pump 
strength

$$
P \equiv \frac{1}{4}\left[\hat{\omega}_{B}^{\prime}\left(\hat{\gamma}^{\prime}\right) / \Gamma_{0}^{\prime} c k_{p}^{\prime}\right]^{6}
$$

defined in Eq. $(105)$ is small $(P \ll 1)$, the maximum growth rate is determined analytically [Eq.(110)]. In the definition of the pump strength $P$, the quantity $\hat{\omega}_{B}^{\prime}\left(\dot{\gamma}^{\prime}\right)$ is the bounce frequency of a deeply trapped electron with energy $\hat{\gamma}^{\prime}$ [Eq.(78)], and $\left(3^{1 / 2} / 2\right) \Gamma_{0}^{\prime} c k_{p}^{\prime}$ is the familiar small-signal gain (temporal growth rate $)^{75}$ calculated in the ponderomotive frame.

For a distribution $f^{0}\left(\gamma_{0}^{\prime}\right)$ with only trapped electrons, the dispersion relation [Eq.(64)] can be simplified analytically (Sec.V). Making use of the exact trajectories of the trapped electrons [Eq.(116)], we express the dispersion relation in a form that involves integrals over only the variable $\gamma_{0}^{\prime}$ [Eq.(130)]. To further simplify the analysis, in Sec.VI we assume a step-function distribution [Eq.(132)] in which the electrons uniformly populate the ponderomotive potential up to an energy $\gamma_{M}^{\prime}<\hat{\gamma}_{+}^{\prime}$. The dispersion relation can then be simplified in closed form [Eqs.(139) and (141)]. In the weak-pump limit $(P \ll 1)$, the maximum growth rate is obtained analytically [Eq.(147)]. The dispersion relations (103) and (141) are solved numerically and the results are presented in Sec.VI C. For different values of the dimensionless pump strength $P$, the dependence of stability properties on the level of population $\left[\gamma_{M}^{\prime}\right.$ in Eq.(132)] of the ponderomotive potential by the trapped electrons is examined. In the strong-pump regime $(P \gg 1)$ and in the intermediate-pump regimes $(P \simeq 1)$, a strong resonance structure is observed in plots of the growth rate versus wavenumber. It is also found in these regimes that an increase in $\gamma_{M}^{\prime}$ causes a decrease in the maximum growth rate and a decrease in the separation between growth rate peaks. When $\gamma_{M I}^{\prime}$ approaches $\hat{\gamma}_{+}^{\prime}$, the growth rate peaks coalesce into a single smooth curve. By contrast, the change in $\gamma_{M}^{\prime}$ has little effect on the stability properties in the weak-pump limit $(P \ll 1)$. Moreover, the resonance structure is not observed in this limit. 


\section{Theoretical Model and Evolution Equations}

\section{A. Assumptions and Basic Equations}

The model consists of a tenuous, relativistic electron beam propagating in the $z$ direction through a helical wiggler magnetic field with vector potential

$$
\begin{aligned}
\mathbf{A}_{w}(\mathbf{x}) & =\left(m c^{2} / e\right) \mathbf{a}_{w}(\mathbf{x}) \\
& =-\left(m c^{2} / e\right) a_{w}\left(\cos k_{0} z \hat{\mathbf{e}}_{x}+\sin k_{0} z \hat{\mathbf{e}}_{y}\right)
\end{aligned}
$$

Here $-e$ is the electron charge, $m c^{2}$ is the electron rest energy, $\lambda_{w}=2 \pi / k_{0}=$ const is the wiggler wavelength, the wiggler magnetic field is $\mathrm{B}_{w}=\nabla \times \mathrm{A}_{w}$, and $a_{w}=$ $e \hat{B}_{w} / m c^{2} k_{0}=$ const is the normalized wiggler amplitude. Transverse spatial variations are neglected $(\partial / \partial x=0=\partial / \partial y)$, and it is assumed that the beam density and current are sufficiently low that the equilibrium self fields associated with the space charge and axial current of the electron beam are negligibly small. Moreover, longitudinal perturbations are neglected in the stability analysis (Compton-regime approximation with $\delta \phi \simeq 0$ ). The electromagnetic wave signal is assumed to be right-circularly polarized with vector potential

$$
\begin{aligned}
\mathbf{A}_{s}(\mathbf{x}, t)= & \left(m c^{2} / e\right) \mathbf{a}_{s}(\mathbf{x}, t) \\
= & \left(m c^{2} / e\right) \hat{a}_{s}(z, t)\left\{\cos \left[k_{s} z-\omega_{s} t+\delta_{s}(z, t)\right] \hat{\mathbf{e}}_{x}\right. \\
& \left.-\sin \left[k, z-\omega_{s} t+\delta_{s}(z, t)\right] \hat{\mathbf{e}}_{y}\right\}
\end{aligned}
$$

where $\omega$, and $k$, are the frequency and wavenumber, respectively. In order to take the sideband structure into account, the wave amplitude $\hat{a}_{s}(z, t)$ and phase shift $\delta_{s}(z, t)$ are allowed to vary slowly. The corresponding electromagnetic fields are given by $\mathrm{B}_{s}=\nabla \times \mathrm{A}_{s}$, and $\mathrm{E}_{s}=-(1 / c) \partial \mathrm{A}_{s} / \partial t$. The amplitude $\dot{a}_{s}(z, t)$ in Eq. $(2)$ is related to the magnetic field amplitude $\hat{B}_{s}(z, t)$ of the electromagnetic wave approximately by $\hat{a}_{s}=e \hat{B}_{s} / m c^{2} k$, for slowly varying $\hat{a}_{s}$. 
A detailed investigation of the sideband instability simplifies considerably if the analysis is carried out in the ponderomotive frame moving with velocity ${ }^{21-25,73,74}$

$$
v_{p}=\omega_{s} /\left(k_{s}+k_{0}\right)
$$

Therefore, the subsequent analysis is carried out in ponderomotive-frame variables $\left(z^{\prime}, t^{\prime}, \gamma^{\prime}\right)$ defined by the Lorentz transformation

$$
\begin{aligned}
z^{\prime} & =\gamma_{p}\left(z-v_{p} t\right), \quad t^{\prime}=\gamma_{p}\left(t-v_{p} z / c^{2}\right) \\
\gamma^{\prime} & =\gamma_{p}\left(\gamma-v_{p} p_{z} / m c^{2}\right)
\end{aligned}
$$

where

$$
\begin{aligned}
\gamma_{p} & =\left(1-v_{p}^{2} / c^{2}\right)^{-1 / 2} \\
\gamma^{\prime} m c^{2} & =\left(m^{2} c^{4}+c^{2} p_{x}^{\prime 2}+c^{2} p_{y}^{\prime 2}+c^{2} p_{z}^{\prime 2}\right)^{1 / 2}
\end{aligned}
$$

Here, $\gamma^{\prime} m c^{2}$ is the mechanical energy, and the components of momentum $\left(p_{x}^{\prime}, p_{y}^{\prime}, p_{z}^{\prime}\right)$ are related to the velocity $\mathbf{v}^{\prime}=d \mathbf{x}^{\prime} / d t^{\prime}$ by $\mathrm{p}^{\prime}=\gamma^{\prime} m \mathbf{v}^{\prime}$. We introduce the complex representation of the vector potentials defined by

$$
\begin{aligned}
a_{w}^{-}(z) & =a_{x w}(z)-i a_{y w}(z), \\
a_{s}^{-}(z, t) & =a_{x s}(z, t)-i a_{y s}(z, t) .
\end{aligned}
$$

Making use of Eqs.(1) and (2) and the inverse transformation of Eq.(4), it is readily shown that

$$
\begin{aligned}
& a_{w}^{-}\left(z^{\prime}, t^{\prime}\right)=-a_{w} \exp \left[-i \gamma_{p} k_{0}\left(z^{\prime}+v_{p} t^{\prime}\right)\right] \\
& a_{s}^{-}\left(z^{\prime}, t^{\prime}\right)=\hat{a}_{s} \exp \left[i\left(k_{s}^{\prime} z^{\prime}-\omega_{s}^{\prime} t^{\prime}\right)+i \delta_{s}^{\prime}\left(z^{\prime}, t^{\prime}\right)\right]
\end{aligned}
$$

in ponderomotive frame variables. Here $\left(\omega_{s}^{\prime}, k_{s}^{\prime}\right)$ in the ponderomotive frame is related to $\left(\omega_{s}, k_{s}\right)$ in the laboratory frame by

$$
\omega_{s}^{\prime}=\gamma_{p}\left(\omega_{s}-k_{s} v_{p}\right), \quad k_{s}^{\prime}=\gamma_{p}\left(k_{s}-\omega_{s} v_{p} / c^{2}\right)
$$


For future reference, the second equation in (6) can also be expressed as

$$
a_{s}^{-}\left(z^{\prime}, t^{\prime}\right)=a_{s}\left(z^{\prime}, t^{\prime}\right) \exp \left[i\left(k_{s}^{\prime} z^{\prime}-\omega_{s}^{\prime} t^{\prime}\right)\right]
$$

where the complex amplitude $a_{\mathbf{s}}\left(z^{\prime}, t^{\prime}\right)$ is defined by

$$
a_{s}\left(z^{\prime}, t^{\prime}\right)=\hat{a}_{s}\left(z^{\prime}, t^{\prime}\right) \exp \left[i \delta_{s}^{\prime}\left(z^{\prime}, t^{\prime}\right)\right]
$$

In the present analysis, it is assumed that all electrons move on surfaces with zero transverse canonical momentum. Correspondingly, the one-particle distribution function for the beam electrons, in the ponderomotive frame, is of the form

$$
f_{b}\left(z^{\prime}, \mathbf{p}^{\prime}, t^{\prime}\right)=\delta\left(P_{x}^{\prime}\right) \delta\left(P_{y}^{\prime}\right) f\left(z^{\prime}, p_{z}^{\prime}, t^{\prime}\right)
$$

where $P_{x}^{\prime}$ and $P_{y}^{\prime}$ are the transverse canonical momenta

$$
\begin{aligned}
P_{x}^{\prime} & =p_{x}^{\prime}-\frac{e}{c} A_{w x}\left(z^{\prime}, t^{\prime}\right)-\frac{e}{c} A_{s x}\left(z^{\prime}, t^{\prime}\right) \\
& =p_{x}^{\prime}+m c a_{w} \cos \left[\gamma_{p} k_{0}\left(z^{\prime}+v_{p} t^{\prime}\right)\right]-m c \hat{a}_{s} \cos \left[k_{s}^{\prime} z^{\prime}-\omega_{s}^{\prime} t^{\prime}+\delta_{s}^{\prime}\left(z^{\prime}, t^{\prime}\right)\right] \\
P_{y}^{\prime} & =p_{y}^{\prime}-\frac{e}{c} A_{w y}\left(z^{\prime}, t^{\prime}\right)-\frac{e}{c} A_{s y}\left(z^{\prime}, t^{\prime}\right) \\
& =p_{y}^{\prime}+m c a_{w} \sin \left[\gamma_{p} k_{0}\left(z^{\prime}+v_{p} t^{\prime}\right)\right]+m c \hat{a}_{s} \sin \left[k_{s}^{\prime} z^{\prime}-\omega_{s}^{\prime} t^{\prime}+\delta_{s}^{\prime}\left(z^{\prime}, t^{\prime}\right)\right]
\end{aligned}
$$

In the present field configuration, $P_{x}^{\prime}$ and $P_{y}^{\prime}$ are exact constants of the motion in the combined fields of the wiggler [Eq.(1)] and the signal wave [Eq.(2)]. For the class of distribution functions in Eq.(10), the transverse velocities can be expressed as

$$
\begin{aligned}
& v_{x}^{\prime}=-\frac{c}{\gamma^{\prime}}\left\{a_{w} \cos \left[\gamma_{p} k_{0}\left(z^{\prime}+v_{p} t^{\prime}\right)\right]-\hat{a}_{s} \cos \left[k_{s}^{\prime} z^{\prime}-\omega_{s}^{\prime} t^{\prime}+\delta_{s}^{\prime}\left(z^{\prime}, t^{\prime}\right)\right]\right\} \\
& v_{y}^{\prime}=-\frac{c}{\gamma^{\prime}}\left\{a_{w} \sin \left[\gamma_{p} k_{0}\left(z^{\prime}+v_{p} t^{\prime}\right)\right]+\dot{a}_{s} \sin \left[k_{s}^{\prime} z^{\prime}-\omega_{s}^{\prime} t^{\prime}+\delta_{s}^{\prime}\left(z^{\prime}, t^{\prime}\right)\right]\right\}
\end{aligned}
$$

where the relativistic mass factor $\gamma^{\prime}$ is given by

$$
\gamma^{\prime 2}=1+\frac{p_{z}^{\prime 2}}{m^{2} c^{2}}+a_{w}^{2}+\left|a_{s}\right|^{2}-2 a_{w} \operatorname{Re}\left[a_{s} \exp \left(i k_{p}^{\prime} z^{\prime}\right)\right]
$$


and $k_{p}^{\prime}$ is defined by

$$
k_{p}^{\prime} \equiv k_{s}^{\prime}+\gamma_{p} k_{0}=\left(k_{s}+k_{0}\right) / \gamma_{p}
$$

In obtaining Eqs.(13) and (14), use is made of Eqs.(3) and (7). The one-dimensional distribution function $f\left(z^{\prime}, p_{z}^{\prime}, t^{\prime}\right)$ in Eq.(10) satisfies the nonlinear Vlasov equation

$$
\frac{\partial f}{\partial t^{\prime}}+m c^{2} \frac{\partial \gamma^{\prime}}{\partial p_{z}^{\prime}} \frac{\partial f}{\partial z^{\prime}}-m c^{2} \frac{\partial \gamma^{\prime}}{\partial z^{\prime}} \frac{\partial f}{\partial p_{z}^{\prime}}=0
$$

where the electron Hamiltonian $\gamma^{\prime} m c^{2}$ is defined by Eq.(13).

To complete the theoretical model, the space-time evolution of $a_{s}^{-}\left(z^{\prime}, t^{\prime}\right)$ is determined self-consistently from Maxwell's equations. For the class of distribution functions in Eq.(10), Maxwell's equations for the complex vector potential $a_{s}^{-}\left(z^{\prime}, t^{\prime}\right)=$ $a_{x}\left(z^{\prime}, t^{\prime}\right)-i a_{y}\left(z^{\prime}, t^{\prime}\right)$ associated with the electromagnetic fields of the signal wave can be expressed as

$$
\left(\frac{1}{c^{2}} \frac{\partial^{2}}{\partial t^{\prime 2}}-\frac{\partial^{2}}{\partial z^{\prime 2}}\right) a_{s}^{-}\left(z^{\prime}, t^{\prime}\right)=-\frac{4 \pi e^{2}}{m c^{3}} \int_{-\infty}^{\infty} d p_{z}^{\prime} f\left(z^{\prime}, p_{z}^{\prime}, t^{\prime}\right)\left(v_{x}^{\prime}-i v_{y}^{\prime}\right)
$$

where the transverse velocities $v_{x}^{\prime}$ and $v_{y}^{\prime}$ are defined in Eq.(12). Making use of Eqs.(8) and (12), it follows that Eq.(16) can be expressed in the equivalent form

$$
\begin{aligned}
{\left[\left(-\frac{\omega_{s}^{\prime 2}}{c^{2}}+k_{s}^{\prime 2}\right)\right.} & \left.a_{s}+\left(\frac{1}{c^{2}} \frac{\partial^{2} a_{s}}{\partial t^{\prime 2}}-\frac{\partial^{2} a_{s}}{\partial z^{\prime 2}}\right)-\frac{2 i \omega_{s}^{\prime}}{c^{2}}\left(\frac{\partial a_{s}}{\partial t^{\prime}}+\frac{c^{2} k_{s}^{\prime}}{\omega_{s}^{\prime}} \frac{\partial a_{s}}{\partial z^{\prime}}\right)\right] \exp \left[i\left(k_{s}^{\prime} z^{\prime}-\omega_{s}^{\prime} t^{\prime}\right)\right] \\
= & -\frac{4 \pi e^{2}}{m c^{2}} \int_{-\infty}^{\infty} d p_{z}^{\prime} f\left(z^{\prime}, p_{z}^{\prime}, t^{\prime}\right) \frac{1}{\gamma^{\prime}} a_{s} \exp \left[i\left(k_{s}^{\prime} z^{\prime}-\omega_{s}^{\prime} t^{\prime}\right)\right] \\
& +\frac{4 \pi e^{2} a_{w}}{m c^{2}} \int_{-\infty}^{\infty} d p_{z}^{\prime} f\left(z^{\prime}, p_{z}^{\prime}, t^{\prime}\right) \frac{1}{\gamma^{\prime}} \exp \left[-i k_{0} \gamma_{p}\left(z^{\prime}+v_{p} t^{\prime}\right)\right]
\end{aligned}
$$

Consistent with the assumption that the complex amplitude $a_{s}$ is slowly varying with $z^{\prime}$ and $t^{\prime}$, we neglect the second-derivative contributions with respect to $z^{\prime}$ and $t^{\prime}$ in Eq.(17), but retain the terms proportional to $\partial a_{s} / \partial t^{\prime}$ and $\partial a_{s} / \partial z^{\prime}$ (eikonal approximation). Furthermore, we operate on Eq.(17) with $\frac{1}{L^{\prime}} \int_{0}^{L^{\prime}} d z^{\prime} \exp \left(-i k_{s}^{\prime} z^{\prime}+i \omega_{s}^{\prime} t^{\prime}\right)$, where $L^{\prime}=2 \pi / k_{p}^{\prime}[$ Eq.(14)] is the fundamental periodicity length for the (fast) spatial 
oscillations in the ponderomotive frame. Treating the spatial variation of $a_{\text {s }}$ as slow, the wave equation (17) can be approximated by

$$
\begin{aligned}
{\left[\left(-\frac{\omega_{s}^{\prime 2}}{c^{2}}+k_{s}^{\prime 2}\right.\right.} & \left.+\frac{4 \pi e^{2}}{m c^{2}} \frac{1}{L^{\prime}} \int_{0}^{L^{\prime}} d z^{\prime} \int_{-\infty}^{\infty} d p_{z}^{\prime} f\left(z^{\prime}, p_{z}^{\prime}, t^{\prime}\right) \frac{1}{\gamma^{\prime}}\right) a \\
& \left.-\frac{2 i \omega_{s}^{\prime}}{c^{2}}\left(\frac{\partial a_{s}}{\partial t^{\prime}}+\frac{c^{2} k_{s}^{\prime}}{\omega_{s}^{\prime}} \frac{\partial a_{s}}{\partial z_{s}^{\prime}}\right)\right] \\
& =\frac{4 \pi e^{2} a_{w}}{m c^{2}} \frac{1}{L^{\prime}} \int_{0}^{L^{\prime}} d z^{\prime} f\left(z^{\prime}, p_{z}^{\prime}, t^{\prime}\right) \frac{\exp \left(-i k_{p}^{\prime} z^{\prime}\right)}{\gamma^{\prime}}
\end{aligned}
$$

for the evolution of the signal wave. Use has been made of $\omega_{s}^{\prime}=\gamma_{p} k_{0} v_{p}$ to obtain Eq.(18). Separating Eq.(18) into fast and slow contributions gives

$$
\begin{aligned}
& \omega_{s}^{\prime 2}=c^{2} k_{s}^{\prime 2}+\frac{4 \pi e^{2}}{m} \frac{1}{L^{\prime}} \int_{0}^{L^{\prime}} d z^{\prime} \int_{-\infty}^{\infty} d p_{z}^{\prime} f\left(z^{\prime}, p_{z}^{\prime}, t^{\prime}\right) \frac{1}{\gamma^{\prime}\left(z^{\prime}, p_{z}^{\prime}\right)} \\
& \frac{2 i \omega_{s}^{\prime}}{c^{2}}\left(\frac{\partial a_{s}}{\partial t^{\prime}}+\frac{k_{s}^{\prime} c^{2}}{\omega_{s}^{\prime}} \frac{\partial a_{s}}{\partial z^{\prime}}\right) \\
& =-\frac{4 \pi e^{2} a_{w}}{m c^{2}} \frac{1}{L^{\prime}} \int_{0}^{L^{\prime}} d z^{\prime} \int_{-\infty}^{\infty} d p_{z}^{\prime} f\left(z^{\prime}, p_{z}^{\prime}, t^{\prime}\right) \frac{\exp \left(-i k_{p}^{\prime} z^{\prime}\right)}{\gamma^{\prime}\left(z^{\prime}, p_{z}^{\prime}\right)}
\end{aligned}
$$

The first equation in (19) determines the real oscillation frequency $\omega_{s}^{\prime}$ in terms of $k_{,}^{\prime}$ and beam dielectric effects. On the other hand, the second equation in (19) describes the (slow) evolution of the complex amplitude $a_{s}\left(z^{\prime}, t^{\prime}\right)=\hat{a}_{s}\left(z^{\prime}, t^{\prime}\right) \exp \left[i \delta_{s}^{\prime}\left(z^{\prime}, t^{\prime}\right)\right]$ induced by the wiggler field.

\section{B. Equilibrium Analysis}

Under quasi-steady-state conditions, an equilibrium analysis of Eqs.(15) and (19) proceeds by setting $\frac{\partial}{\partial t^{\prime}}=0$ and looking for stationary solutions, $\hat{n}_{b}^{\prime} f^{0}\left(z^{\prime}, p_{z}^{\prime}\right)$ and $a_{s}^{0}\left(z^{\prime}\right)$, that satisfy the equations

$$
\begin{aligned}
& m c^{2} \frac{\partial \gamma_{0}^{\prime}}{\partial p_{z}^{\prime}} \frac{\partial f^{0}}{\partial z^{\prime}}-m c^{2} \frac{\partial \gamma_{0}^{\prime}}{\partial z^{\prime}} \frac{\partial f^{0}}{\partial p_{z}^{\prime}}=0 \\
& \omega_{s}^{\prime 2}=c^{2} k_{s}^{\prime 2}+\frac{4 \pi e^{2}}{m} \frac{\hat{n}_{b}^{\prime}}{L^{\prime}} \int_{0}^{L^{\prime}} d z^{\prime} \int_{-\infty}^{\infty} d p_{z}^{\prime} f^{0}\left(z^{\prime}, p_{z}^{\prime}, t^{\prime}\right) \frac{1}{\gamma^{\prime}\left(z^{\prime}, p_{z}^{\prime}\right)} \\
& 2 i k_{s}^{\prime} \frac{\partial a_{s}^{0}}{\partial z^{\prime}}=-\frac{4 \pi e^{2} a_{w}}{m c^{2}} \frac{\hat{n}_{b}^{\prime}}{L^{\prime}} \int_{0}^{L^{\prime}} d z^{\prime} \int_{-\infty}^{\infty} d p_{z}^{\prime} f^{0}\left(z^{\prime}, p_{z}^{\prime}, t^{\prime}\right) \frac{\exp \left(-i k_{p}^{\prime} z^{\prime}\right)}{\gamma^{\prime}\left(z^{\prime}, p_{z}^{\prime}\right)}
\end{aligned}
$$


where $\gamma_{0}^{\prime} m c^{2}$ is the Hamiltonian under the influence of the wiggler field $a_{w}$ and the stationary electromagnetic wave field $a_{s}^{0}\left(z^{\prime}\right)$. Furthermore, $\hat{n}_{b}^{\prime}=$ const is the average density of the beam electrons in the ponderomotive frame, which is chosen so that the normalization condition $\frac{1}{L^{\prime}} \int_{0}^{L^{\prime}} d z^{\prime} \int_{-\infty}^{\infty} d p_{z}^{\prime} f^{0}\left(z^{\prime}, p_{z}^{\prime}, t^{\prime}\right)=1$ is satisfied. Similar to Eq. (13), $\gamma_{0}^{\prime}$ is given by

$$
\gamma_{0}^{\prime 2}=1+\frac{p_{z}^{\prime 2}}{m^{2} c^{2}}+a_{w}^{2}+\left|a_{s}^{0}\right|^{2}-2 a_{w} \operatorname{Re}\left[a_{s}^{0} \exp \left(i k_{p}^{\prime} z^{\prime}\right)\right]
$$

It is clear that any distribution function which depends only on the constant of the motion, $\gamma_{0}^{\prime}\left(z^{\prime}, p_{z}^{\prime}\right)$, solves the first equation in (20). The complex amplitude $a_{s}^{0}\left(z^{\prime}\right)$ of the electromagnetic wave can be determined by solving the third equation in (20). In the present analysis, it is assumed that in the equilibrium state the electromagnetic wave has constant amplitude and slowly varying phase, i.e.,

$$
a_{,}^{0}\left(z^{\prime}\right)=\hat{a}_{,}^{0} \exp \left[i \delta_{s}^{0}\left(z^{\prime}\right)\right]
$$

where $\hat{a}_{s}^{0}=$ const.(independent of $z^{\prime}$ ). Under this assumption, it follows from Eqs.(20) and (21) that

$$
\begin{aligned}
&-2 k_{s}^{\prime} \hat{a}_{s}^{0} \frac{\partial \delta_{s}^{0}}{\partial z^{\prime}}=-\frac{4 \pi e^{2} a_{w}}{m c^{2}} \frac{\hat{n}_{b}^{\prime}}{L^{\prime}} \int_{0}^{L^{\prime}} d z^{\prime} \int_{-\infty}^{\infty} d p_{z}^{\prime} f^{0}\left[\gamma_{0}^{\prime}\left(z^{\prime}, p_{z}^{\prime}\right)\right] \\
& \times \frac{\cos \left[k_{p}^{\prime} z^{\prime}+\delta_{s}^{0}\left(z^{\prime}\right)\right]}{\gamma_{0}^{\prime}\left(z^{\prime}, p_{z}^{\prime}\right)} \\
& \gamma_{0}^{\prime 2}\left(z^{\prime}, p_{z}^{\prime}\right)=1+\frac{p_{z}^{\prime 2}}{m^{2} c^{2}}+a_{w}^{2}+\left(\hat{a}_{s}^{\prime \prime}\right)^{2}-2 a_{w} \hat{a}_{s}^{0} \cos \left[k_{p}^{\prime} z^{\prime}+\delta_{s}^{0}\left(z^{\prime}\right)\right]
\end{aligned}
$$

Note that the integrals over $z^{\prime}$ in Eqs.(20) and (23) are over an interval of length $L^{\prime}=2 \pi / k_{p}^{\prime}$. Moreover, neglecting the slow $z^{\prime}$ variation of $\delta_{s}^{0}\left(z^{\prime}\right)$ in the integration over $z^{\prime}$ in Eqs.(20) and (23), the integrands are periodic functions of $z^{\prime}$ with periud $L^{\prime}=2 \pi / k_{p}^{\prime}$. Therefore, the right-hand side of the second equation in $(20)$ and the first equation in (23) are independent of $z^{\prime}$. The solution of the first equation in (23) is readily given by

$$
\delta_{s}^{0}\left(z^{\prime}\right)=\epsilon k_{p}^{\prime} z^{\prime}
$$


where $\epsilon$ is the small dimensionless parameter defined by

$$
\begin{aligned}
\epsilon k_{p}^{\prime} & =\text { const. } \\
& =\frac{2 \pi e^{2} a_{w}}{k_{s}^{\prime} m c^{2} \hat{a}_{s}^{0}} \frac{\hat{n}_{b}^{\prime}}{L^{\prime}} \int_{0}^{L^{\prime}} d z^{\prime} \int_{-\infty}^{\infty} d p_{z}^{\prime} f^{0}\left[\gamma_{0}^{\prime}\left(z^{\prime}, p_{z}^{\prime}\right)\right] \frac{\cos \left[k_{p}^{\prime} z^{\prime}+\epsilon k_{p}^{\prime} z^{\prime}\right]}{\gamma_{0}^{\prime}\left(z^{\prime}, p_{z}^{\prime}\right)} .
\end{aligned}
$$

Similarly, the second equation in (20) can be expressed as

$$
\omega_{s}^{\prime 2}=c^{2} k_{s}^{\prime 2}+\frac{4 \pi e^{2}}{m} \frac{\hat{n}_{b}^{\prime}}{L^{\prime}} \int_{0}^{L^{\prime}} d z^{\prime} \int_{-\infty}^{\infty} d p_{z}^{\prime} f^{0}\left[\gamma_{0}^{\prime}\left(z^{\prime}, p_{z}^{\prime}\right)\right] \frac{1}{\gamma_{0}^{\prime}\left(z^{\prime}, p_{z}^{\prime}\right)}
$$

which plays the role of a dispersion relation relating $\omega_{s}^{\prime}$ and $k_{s}^{\prime}$, including beam dielectric effects. For future reference, we differentiate $\mathrm{Eq} .(26)$ with respect to $k_{s}^{\prime}$, and obtain the group velocity of the electromagnetic wave in the ponderomotive frame

$$
v_{g}^{\prime}=\frac{\partial \omega_{s}^{\prime}}{\partial k_{s}^{\prime}}=\frac{k_{s}^{\prime} c^{2}}{\omega_{s}^{\prime}}
$$

Several noteworthy points regarding the equilibrium analysis are summarized below:

(a) For constant equilibrium wave amplitude $\hat{a}_{s}^{0}$, the wave phase $\delta_{s}^{0}\left(z^{\prime}\right)$ is generally slowly varying in order to satisfy the third equation in (20). The equilibrium phase shift is given by Eqs.(24) and (25).

(b) We note from Eq.(23) that the fundamental periodicity of the (fast) spatial oscillation is actually given by $L^{\prime}=2 \pi /\left[(1+\epsilon) k_{p}^{\prime}\right]$. Since the present model assumes that the phase varies slowly, i.e., $|\epsilon| \ll 1$, there is only a small correction to the previously defined $L^{\prime}=2 \pi / k_{p}^{\prime}$.

(c) In Eq.(25), $\epsilon k_{p}^{\prime}$ appears on both sides of the equation. It would seem necessary to determine $\epsilon k_{\mathrm{p}}^{\prime}$ in an implicit manner. However, consistent with the assumption that $\epsilon \ll 1$, we can approximate Eq. $(25)$ by

$$
\epsilon k_{p}^{\prime}=\frac{2 \pi e^{2} a_{w}}{k_{s}^{\prime} m c^{2} \hat{a}_{s}^{0}} \frac{\hat{n}_{b}^{\prime}}{2 \pi} \int_{0}^{2 \pi} d\left(k_{p}^{\prime} z^{\prime}\right) \int_{-\infty}^{\infty} d p_{z}^{\prime} f^{0}\left[\gamma_{0}^{\prime}\left(z^{\prime}, p_{z}^{\prime}\right)\right] \frac{\cos \left(k_{p}^{\prime} z^{\prime}\right)}{\gamma_{0}^{\prime}\left(z^{\prime}, p_{z}^{\prime}\right)},
$$


where the relativistic mass factor $\gamma_{0}^{\prime}\left(z^{\prime}, p_{z}^{\prime}\right)$ is given approximately by

$$
\gamma_{0}^{\prime 2}\left(z^{\prime}, p_{z}^{\prime}\right)=1+\frac{p_{z}^{\prime 2}}{m^{2} c^{2}}+a_{w}^{2}+\left(\hat{a}_{s}^{0}\right)^{2}-2 a_{w} \hat{a}_{s}^{0} \cos \left(k_{p}^{\prime} z^{\prime}\right) .
$$

Note that in Eq.(28), $k_{p}^{\prime} z^{\prime}$ is a dummy variable. Therefore, $\epsilon k_{p}^{\prime}$ is determined explicitly.

(d) Sulving Eq.(29) for $p_{z}^{\prime}$ gives

$$
p_{z}^{\prime}= \pm m c\left[\gamma_{0}^{\prime 2}-1-a^{2}\left(z^{\prime}\right)\right]^{1 / 2}
$$

where $a^{2}\left(z^{\prime}\right)$ is defined by

$$
a^{2}\left(z^{\prime}\right)=a_{w}^{2}+\left(\hat{a}_{s}^{0}\right)^{2}-2 a_{w} \hat{a}_{s}^{0} \cos \left(k_{p}^{\prime} z^{\prime}\right) .
$$

It is clear from Eqs (29), (30) and (31) that there are two classes of electron orbits. Untrapped electrons, for which $p_{z}^{\prime}$ does not change sign, have energy $\gamma_{0}^{\prime} m c^{2}$ satisfying (Fig.1)

$$
\gamma_{0}^{\prime}>\left[1+\left(a_{w}+\hat{a}_{s}^{0}\right)^{2}\right]^{1 / 2} \equiv{\hat{\gamma^{\prime}}}_{+},
$$

where $\hat{a}_{s}^{0}>0$ and $a_{w}>0$ are assumed without loss of generality. On the other hand, trapped electrons, which execute periodic motion in the ponderomotive potential, have energy $\gamma_{0}^{\prime} m c^{2}$ in the range (Fig.1)

$$
\gamma_{-}^{\prime}\left(z^{\prime}\right) \equiv\left[1+a^{2}\left(z^{\prime}\right)\right]^{1 / 2}<\gamma_{0}^{\prime}<\gamma_{+}^{\prime} .
$$

For the trapped electrons, the density of particles with positive and negative momentum in the ponderomotive frame must be identical. Therefore $f^{0}\left(z^{\prime}, p_{z}^{\prime}\right)=f^{0}\left(\gamma_{0}^{\prime}\right)$ is a complete description for the trapped electron distribution. However, for untrapped electrons the complete form of the distribution function is

$$
f_{u}^{0}\left(z^{\prime}, p_{z}^{\prime}\right)=f_{>}^{0}\left(\gamma_{0}^{\prime}\right) U\left(p_{z}^{\prime}\right)+f_{<}^{0}\left(\gamma_{0}^{\prime}\right) U\left(-p_{z}^{\prime}\right)
$$

where $U(x)$ is the Heaviside step function defined by $U(x)=1$ for $x \geq 0$ and $U(x)=0$ for $x<0$. In Eq. $(34), f_{>}^{0}\left(\gamma_{0}^{\prime}\right)$ and $f_{<}^{0}\left(\gamma_{0}^{\prime}\right)$ are the distribution functions of the forwardmoving $\left(p_{z}^{\prime}>0\right)$ and backward-moving $\left(p_{z}^{\prime}<0\right)$ electrons in the ponderomotive frame. For the untrapped electrons, $f_{>}^{0}\left(\gamma_{0}^{\prime}\right)$ and $f_{<}^{0}\left(\gamma_{0}^{\prime}\right)$ can be specified independently. 
(e) Although the present analysis requires that the equilibrium distribution $f^{0}\left(z^{\prime}, p_{z}^{\prime}\right)$ be a function of $\gamma_{0}^{\prime}$, it provides no information about the detailed dependence of $f^{0}$ on $\gamma_{0}^{\prime}$. Generally speaking, we should account for the system evolution from some initial state $f\left(z^{\prime}, p_{z}^{\prime}, t^{\prime}=0\right)$ to the quasi-steady state in Eq. $(20)$ in order to determine the actual form of $f^{0}\left(\gamma_{0}^{\prime}\right)$. For present purposes, we first carry out a linear stability analysis for perturbations about general $f^{0}\left(\gamma_{0}^{\prime}\right)$. Later, when the form of $f^{0}\left(\gamma_{0}^{\prime}\right)$ is required to determine detailed stability behavior, a choice of $f^{0}\left(\gamma_{0}^{\prime}\right)$ will be made which makes the analysis tractable. 


\section{Linear Stability Analysis}

\section{A. Linearized Vlasov-Maxwell Equations}

With regard to a stability analysis based on the Vlasov-Maxwell equations (15) and (19), we consider small-amplitude perturbation about the quasi-steady state $f^{0}\left(z^{\prime}, p_{z}^{\prime}\right)$ and $\hat{a}_{s}^{0} \exp \left[i \delta_{s}^{0}\left(z^{\prime}\right)\right]$ described in Sec.II $B$, and express

$$
\begin{aligned}
f\left(z^{\prime}, p_{z}^{\prime}, t^{\prime}\right) & =\hat{n}_{b}^{\prime}\left[f^{0}\left(z^{\prime}, p_{z}^{\prime}\right)+\delta f\left(z^{\prime}, p_{z}^{\prime}, t^{\prime}\right)\right] \\
a_{s}\left(z^{\prime}, t^{\prime}\right) & =\left[\hat{a}_{s}^{0}+\delta a_{s}\left(z^{\prime}, t\right)\right] \exp \left[i \delta_{s}^{0}\left(z^{\prime}\right)\right]
\end{aligned}
$$

where $\hat{n}_{b}^{\prime}=$ const is the average density of the beam electrons in the ponderomotive frame. Here, for small perturbations in wave amplitude $\delta \hat{a}_{s} \equiv \hat{a}_{s}-\hat{a}_{s}^{0}$ and phase $\tilde{\delta}_{s}^{\prime} \equiv \delta_{s}^{\prime}-\delta_{s}^{0}$, it follows that the complex perturbation $\delta a,\left(z^{\prime}, t^{\prime}\right)$ in Eq.(35) can be approximated by $\delta a_{s}=\delta \hat{a}_{s}+i \bar{\delta}_{s}^{\prime} \hat{a}_{s}^{0}$. The linearized Vlasov equation is given by

$$
\begin{aligned}
\left\{\frac{\partial}{\partial t^{\prime}}+m c^{2} \frac{\partial \gamma_{0}^{\prime}}{\partial p_{z}^{\prime}}\right. & \left.\frac{\partial}{\partial z^{\prime}}-m c^{2} \frac{\partial \gamma_{0}^{\prime}}{\partial z^{\prime}} \frac{\partial}{\partial p_{z}^{\prime}}\right\} \delta f\left(z^{\prime}, p_{z}^{\prime}, t^{\prime}\right) \\
& =m c^{2}\left\{\frac{\partial f^{0}}{\partial p_{z}^{\prime}} \frac{\partial}{\partial z^{\prime}}-\frac{\partial f^{0}}{\partial z^{\prime}} \frac{\partial}{\partial p_{z}^{\prime}}\right\} \delta \gamma^{\prime}\left(z^{\prime}, p_{z}^{\prime}\right) .
\end{aligned}
$$

Here, $\delta \gamma=\gamma^{\prime}-\gamma_{0}^{\prime}$, where $\gamma^{\prime}$ and $\gamma_{0}^{\prime}$ are defined in Eqs.(13) and (29), respectively. Making use of Eqs. (28), (29) and (35), we can express Eq.(13) as

$$
\gamma^{\prime 2}=\gamma_{0}^{\prime 2}+2 \hat{a}_{s}^{0} \operatorname{Re}\left(\delta a_{s}\right)+\left|\delta a_{s}\right|^{2}-2 a_{w} \operatorname{Re}\left[\delta a_{s} \exp \left(i k_{\mathrm{p}}^{\prime} z^{\prime}\right)\right]
$$

Consistent with the assumption that $\left|\hat{a}_{s}^{0}\right| \ll 1$ and $\left|\delta a_{s}\right| \ll 1$, we approximate $\delta \gamma^{\prime}$ by

$$
\delta \gamma^{\prime} \simeq-\frac{a_{w}}{\gamma_{0}^{\prime}} \operatorname{Re}\left[\delta a, \exp \left(i k_{p}^{\prime} z^{\prime}\right)\right]
$$

The perturbed complex wave amplitude $\delta a$, is then determined self-consistently from the linearized equation

$$
\begin{aligned}
& 2 \omega_{s}^{\prime}\left(\frac{\partial}{\partial t^{\prime}}+v_{g}^{\prime} \frac{\partial}{\partial z^{\prime}}+i v_{g}^{\prime} \epsilon k_{\mathrm{p}}^{\prime}\right) \delta a_{s} \\
& \quad=i \frac{4 \pi e^{2} a_{\psi}}{m} \frac{\hat{n}_{b}^{\prime}}{L^{\prime}} \int_{0}^{L^{\prime}} d z^{\prime} \int_{-\infty}^{\infty} d p_{z}^{\prime} \delta f\left(z^{\prime}, p_{z}^{\prime}, t^{\prime}\right) \frac{\exp \left[-i k_{\mathrm{p}}^{\prime} z^{\prime}-i \delta_{s}^{o}\left(z^{\prime}\right)\right]}{\gamma^{\prime}\left(z^{\prime}, p_{z}^{\prime}\right)} .
\end{aligned}
$$


In deriving Eq.(39) from Eqs.(19), (27) and (35), zeroth-order terms are eliminated by using Eq.(23).

Equations (36) and (39) can used to determine the evolution of $\delta f$ and $\delta a_{\text {s }}$ selfconsistently. If the perturbations $\delta f$ and $\delta a$, grow, then the equilibrium distribution $f^{0}$ is unstable. If the perturbations damp, the system returns to equilibrium and is stable. A useful method for solving the linearized Vlasov equation (36) is based on the method of characteristics. We denote by $z^{\prime \prime}\left(t^{\prime \prime}\right)$ and $p_{z}^{\prime \prime}\left(t^{\prime \prime}\right)$ the particle trajectories under the influence of the wiggler field and the equilibrium electromagnetic field. That is, $z^{\prime \prime}\left(t^{\prime \prime}\right)$ and $p_{z}^{\prime \prime}\left(t^{\prime \prime}\right)$ satisfy the Hamilton equations of motion

$$
\begin{aligned}
\frac{d}{d t^{\prime \prime}} z^{\prime \prime} & =m c^{2} \frac{\partial}{\partial p_{z}^{\prime \prime}} \gamma_{0}^{\prime}\left(z^{\prime \prime}, p_{z}^{\prime \prime}\right), \\
\frac{d}{d t^{\prime \prime}} p_{z}^{\prime \prime} & =-m c^{2} \frac{\partial}{\partial z^{\prime \prime}} \gamma_{0}^{\prime}\left(z^{\prime \prime}, p_{z}^{\prime \prime}\right),
\end{aligned}
$$

where the Hamiltonian $m c^{2} \gamma_{0}^{\prime}$ is given in Eq.(29). We further assume that the trajectories $z^{\prime \prime}\left(t^{\prime \prime}\right)$ and $p_{z}^{\prime \prime}\left(t^{\prime \prime}\right)$ pass through the phase-space point $\left(z^{\prime}, p_{z}^{\prime}\right)$ at time $t^{\prime \prime}=t^{\prime}$, i.e.,

$$
\begin{aligned}
& z^{\prime \prime}\left(t^{\prime \prime}=t^{\prime}\right)=z^{\prime}, \\
& p_{z}^{\prime \prime}\left(t^{\prime \prime}=t^{\prime}\right)=p_{z}^{\prime} .
\end{aligned}
$$

Using the chain rule for differentiation, it can be shown that the total time derivative, following the particle trajectories in the equilibrium field configuration, is

$$
\begin{aligned}
\frac{d}{d t^{\prime \prime}} & =\frac{\partial}{\partial t^{\prime \prime}}+\frac{d z^{\prime \prime}}{d t^{\prime \prime}} \frac{\partial}{\partial z^{\prime \prime}}+\frac{d p_{z}^{\prime \prime}}{d t^{\prime \prime}} \frac{\partial}{\partial p_{z}^{\prime \prime}} \\
& =\frac{\partial}{\partial t^{\prime \prime}}+m c^{2} \frac{\partial \gamma_{0}^{\prime}}{\partial p_{z}^{\prime \prime}} \frac{\partial}{\partial z^{\prime \prime}}-m c^{2} \frac{\partial \gamma_{0}^{\prime}}{\partial t^{\prime \prime}} \frac{\partial}{\partial p_{z}^{\prime \prime}}
\end{aligned}
$$

Moreover, making use of the fact that the equilibrium distribution function is a function only of $\gamma_{0}^{\prime}$, the following identity is readily shown

$$
\frac{\partial f^{0}}{\partial p_{z}^{\prime \prime}} \frac{\partial}{\partial z^{\prime \prime}}-\frac{\partial f^{0}}{\partial z^{\prime \prime}} \frac{\partial}{\partial p_{z}^{\prime \prime}}=\frac{\partial f^{0}}{\partial \gamma_{0}^{\prime}}\left\{\frac{\partial \gamma_{0}^{\prime}}{\partial p_{z}^{\prime \prime}} \frac{\partial}{\partial z^{\prime \prime}}-\frac{\partial \gamma_{0}^{\prime}}{\partial z^{\prime \prime}} \frac{\partial}{\partial p_{z}^{\prime \prime}}\right\}=\frac{1}{m c^{2}} \frac{\partial f^{0}}{\partial \gamma_{0}^{\prime}}\left\{\frac{d}{d t^{\prime \prime}}-\frac{\partial}{\partial t^{\prime \prime}}\right\}:
$$


It is then evident that Eq.(36) is equivalent to

$$
\frac{d}{d t^{\prime \prime}} \delta f=\frac{\partial f^{0}}{\partial \gamma_{0}^{\prime}}\left\{\frac{d}{d t^{\prime \prime}}-\frac{\partial}{\partial t^{\prime \prime}}\right\} \delta \gamma^{\prime}
$$

evaluated at $t^{\prime \prime}=t^{\prime}$. For amplifying perturbations that grow temporally, we integrate Eq.(44) from $t^{\prime \prime}=-\infty$ to $t^{\prime \prime}=t^{\prime}$, and neglect the initial perturbation (at $t^{\prime \prime}=-\infty$ ). Making use of Eqs.(42) and (43), this gives

$$
\delta f\left(z^{\prime}, p_{z}^{\prime}, t^{\prime}\right)=\frac{\partial f^{0}}{\partial \gamma_{0}^{\prime}}\left[\delta \gamma^{\prime}\left(z^{\prime}, p_{z}^{\prime}, t^{\prime}\right)-\int_{-\infty}^{t^{\prime}} d t^{\prime \prime} \frac{\partial}{\partial t^{\prime \prime}} \delta \gamma^{\prime}\left(z^{\prime \prime}, p_{z}^{\prime \prime}, t^{\prime \prime}\right)\right]
$$

for the perturbed distribution function $\delta f\left(z^{\prime}, p_{z}^{\prime}, t^{\prime}\right)$. Eq. (45) is then substituted into Eq.(39) to determine the self-consistent evolution of the field perturbation, $\delta a_{s}\left(z^{\prime}, t^{\prime}\right)$, in the small-amplitude regime. Note that the integral over $t^{\prime \prime}$ in Eq.(45) requires a determination from Eq. $(40)$ of the orbits $z^{\prime \prime}\left(t^{\prime \prime}\right)$ and $p_{z}^{\prime \prime}\left(t^{\prime \prime}\right)$ in the equilibrium field configuration.

\section{B. Kinetic Dispersion Relation}

For clarity of derivation, we express the complex perturbed wave amplitude $\delta a_{\mathbf{s}}\left(z^{\prime}, t^{\prime}\right)$ explicitly in terms of its real and imaginary parts

$$
\delta a_{s}\left(z^{\prime}, t^{\prime}\right)=\delta a_{s}^{r}\left(z^{\prime}, t^{\prime}\right)+i \delta a_{s}^{i}\left(z^{\prime}, t^{\prime}\right)
$$

where $\delta a_{s}^{r}=\delta \hat{a}_{s} \equiv \hat{a}_{s}-\hat{a}_{s}^{0}$ and $\delta a_{s}^{i}=\bar{\delta}_{s}^{\prime} \hat{a}_{s}^{0} \equiv\left(\delta_{s}^{\prime}-\delta_{s}^{0}\right) \hat{a}_{s}^{0}$. The linearized equation (39) can then be expressed as

$$
\begin{aligned}
& 2 \omega_{s}^{\prime}\left[\left(\frac{\partial}{\partial t^{\prime}}+v_{g}^{\prime} \frac{\partial}{\partial z^{\prime}}\right) \delta a_{s}^{r}-v_{g}^{\prime} \epsilon k_{p}^{\prime} \delta a_{s}^{i}\right] \\
& \quad=\frac{4 \pi e^{2} a_{w}}{m} \frac{\hat{n}_{b}^{\prime}}{2 \pi} \int_{0}^{2 \pi} d\left(k_{p}^{\prime} z^{\prime}\right) \int_{-\infty}^{\infty} d p_{z}^{\prime} \delta f\left(z^{\prime}, p_{z}^{\prime}, t^{\prime}\right) \frac{\sin \left(k_{p}^{\prime} z^{\prime}\right)}{\gamma^{\prime}\left(z^{\prime}, p_{z}^{\prime}\right)} \\
& 2 \omega_{s}^{\prime}\left[\left(\frac{\partial}{\partial t^{\prime}}+v_{g}^{\prime} \frac{\partial}{\partial z^{\prime}}\right) \delta a_{s}^{i}+v_{g}^{\prime} \epsilon k_{p}^{\prime} \delta a_{s}^{r}\right] \\
& =\frac{4 \pi e^{2} a_{w}}{m} \frac{\hat{n}_{b}^{\prime}}{2 \pi} \int_{0}^{2 \pi} d\left(k_{p}^{\prime} z^{\prime}\right) \int_{-\infty}^{\infty} d p_{z}^{\prime} \delta f\left(z^{\prime}, p_{z}^{\prime}, t^{\prime}\right) \frac{\cos \left(k_{p}^{\prime} z^{\prime}\right)}{\gamma^{\prime}\left(z^{\prime}, p_{z}^{\prime}\right)}
\end{aligned}
$$


In obtaining Eq.(47), use is made of the assumption $k_{p}^{\prime} \gg \epsilon k_{p}^{\prime}$ to approximate $k_{p}^{\prime} z^{\prime}+$ $\delta_{s}^{0}\left(z^{\prime}\right)$ by $k_{p}^{\prime} z^{\prime}$. Making use of Eqs.(38) and (46) in Eq.(45), we obtain the expression for $\delta f$ in terms of $\delta a_{s}^{r}$ and $\delta a_{s}^{i}$, i.e.,

$$
\begin{aligned}
& \delta f\left(z^{\prime}, p_{z}^{\prime}, t^{\prime}\right)=-\frac{a_{w}}{\gamma_{0}^{\prime}} \frac{\partial f^{0}}{\partial \gamma_{0}^{\prime}}\left\{\delta a_{s}^{r} \cos \left(k_{p}^{\prime} z^{\prime}\right)-\delta a_{s}^{i} \sin \left(k_{p}^{\prime} z^{\prime}\right)-\int_{-\infty}^{t^{\prime}} d t^{\prime \prime}\right. \\
& \left.\times\left[\left(\frac{\partial}{\partial t^{\prime \prime}} \delta a_{s}^{r}\left(z^{\prime \prime}, t^{\prime \prime}\right)\right) \cos \left(k_{p}^{\prime} z^{\prime \prime}\right)-\left(\frac{\partial}{\partial t^{\prime \prime}} \delta a_{s}^{i}\left(z^{\prime \prime}, t^{\prime \prime}\right)\right) \sin \left(k_{p}^{\prime} z^{\prime \prime}\right)\right]\right\} .
\end{aligned}
$$

In terms of the Fourier-Laplace transforms of $\delta a_{s}^{r}$ and $\delta a_{s}^{i}$, Eq.(48) can be expressed as

$$
\begin{aligned}
\delta f\left(z^{\prime}, p_{z}^{\prime}, t^{\prime}\right)= & -\frac{a_{w}}{\gamma_{0}^{\prime}} \frac{\partial f^{0}}{\partial \gamma_{0}^{\prime}} \int \frac{d\left(\Delta k^{\prime}\right)}{2 \pi} \int_{C} \frac{d\left(\Delta \omega^{\prime}\right)}{2 \pi}\left\{\delta A^{r}\left(\Delta k^{\prime}, \Delta \omega^{\prime}\right) \cos \left(k_{p}^{\prime} z^{\prime}\right)\right. \\
& -\delta A^{i}\left(\Delta k^{\prime}, \Delta \omega^{\prime}\right) \sin \left(k_{p}^{\prime} z^{\prime}\right)-\int_{-\infty}^{t} d t^{\prime \prime} \exp \left[-i \Delta \omega^{\prime}\left(t^{\prime \prime}-t^{\prime}\right)\right] \\
& \times \exp \left[i \Delta k^{\prime}\left(z^{\prime \prime}-z^{\prime}\right)\right]\left[-i \Delta \omega^{\prime} \delta A^{r}\left(\Delta k^{\prime}, \Delta \omega^{\prime}\right) \cos \left(k_{p}^{\prime} z^{\prime \prime}\right)\right. \\
& \left.\left.+i \Delta \omega^{\prime} \delta A^{i}\left(\Delta k^{\prime}, \Delta \omega^{\prime}\right) \sin \left(k_{p}^{\prime} z^{\prime \prime}\right)\right]\right\} \exp \left[i\left(\Delta k^{\prime} z^{\prime}-\Delta \omega^{\prime} t^{\prime}\right)\right],
\end{aligned}
$$

where the transformed variables $\delta A^{r}\left(\Delta k^{\prime}, \Delta \omega^{\prime}\right)$ and $\delta A^{i}\left(\Delta k^{\prime}, \Delta \omega^{\prime}\right)$ are related to the perturbations $\delta a_{s}^{r}$ and $\delta a_{s}^{i}$ by

$$
\begin{aligned}
& \delta a_{s}^{r}\left(z^{\prime}, t^{\prime}\right)=\int \frac{d\left(\Delta k^{\prime}\right)}{2 \pi} \exp \left(i \Delta k^{\prime} z^{\prime}\right) \int_{C} \frac{d\left(\Delta \omega^{\prime}\right)}{2 \pi} \exp \left(-i \Delta \omega^{\prime} t^{\prime}\right) \delta A^{r}\left(\Delta k^{\prime}, \Delta \omega^{\prime}\right), \\
& \delta a_{s}^{i}\left(z^{\prime}, t^{\prime}\right)=\int \frac{d\left(\Delta k^{\prime}\right)}{2 \pi} \exp \left(i \Delta k^{\prime} z^{\prime}\right) \int_{C} \frac{d\left(\Delta \omega^{\prime}\right)}{2 \pi} \exp \left(-i \Delta \omega^{\prime} t^{\prime}\right) \delta A^{i}\left(\Delta k^{\prime}, \Delta \omega^{\prime}\right) .
\end{aligned}
$$

Here, the integral over $\Delta k^{\prime}$ is along the real $\Delta k^{\prime}$ axis from $\Delta k^{\prime}=-\infty$ to $\Delta k^{\prime}=+\infty$, and the contour $C$, which is parallel to the real $\Delta \omega^{\prime}$ axis, is so chosen such that $\operatorname{Im}\left(\Delta \omega^{\prime}\right)$ is sufficiently large that the Laplace transform

$$
A\left(\Delta k^{\prime}, \Delta \omega^{\prime}\right)=\int_{0}^{\infty} d t^{\prime} \exp \left(i \Delta \omega^{\prime} t^{\prime}\right) A\left(\Delta k^{\prime}, t^{\prime}\right)
$$

converges. Because $\delta a_{s}^{r}\left(z^{\prime}, t^{\prime}\right)$ and $\delta a_{s}^{i}\left(z^{\prime}, t^{\prime}\right)$ are real-valued functions, it follows that

$$
\delta A^{r}\left(-\Delta k^{\prime},-\Delta \omega^{\prime *}\right)=\left[\delta A^{r}\left(\Delta k^{\prime}, \Delta \omega^{\prime}\right)\right]^{*}, \quad \delta A^{i}\left(-\Delta k^{\prime},-\Delta \omega^{\prime *}\right)=\left[\delta A^{i}\left(\Delta k^{\prime}, \Delta \omega^{\prime}\right)\right]^{*} .
$$


Consistent with the assumption that $\delta a_{s}^{r}$ and $\delta a_{s}^{i}$ vary slowly, the integrals over $\Delta k^{\prime}$ and $\Delta \omega^{\prime}$ in Eq.(49) contribute mainly from the region satisfying $\left|\Delta k^{\prime}\right| \ll k_{p}^{\prime}$ and $\left|\Delta \omega^{\prime}\right| \ll c k_{p}^{\prime}$ in $\Delta k^{\prime}-\Delta \omega^{\prime}$ space. Therefore, the factor $\exp \left[i \Delta k^{\prime}\left(z^{\prime \prime}-z^{\prime}\right)\right]$ in Eq.(49) varies slowly compared to $\sin \left(k_{p}^{\prime} z^{\prime \prime}\right)$ and $\cos \left(k_{p}^{\prime} z^{\prime \prime}\right)$, so that $\exp \left[i \Delta k^{\prime}\left(z^{\prime \prime}-z^{\prime}\right)\right]$ can be approximated by unity. Equation (49) can then be expressed as

$$
\delta f\left(z^{\prime}, p_{z}^{\prime}, t^{\prime}\right)=\int \frac{d\left(\Delta k^{\prime}\right)}{2 \pi} \int_{C} \frac{d\left(\Delta \omega^{\prime}\right)}{2 \pi} \delta F\left(p_{z}^{\prime}, k_{p}^{\prime} z^{\prime}, \Delta k^{\prime}, \Delta \omega^{\prime}\right) \exp \left[i\left(\Delta k^{\prime} z^{\prime}-\Delta \omega^{\prime} t^{\prime}\right)\right]
$$

where

$$
\begin{aligned}
\delta F\left(p_{z}^{\prime}, k_{p}^{\prime} z^{\prime}, \Delta k^{\prime}, \Delta \omega^{\prime}\right) & =-\frac{a_{w}}{\gamma_{0}^{\prime}} \frac{\partial f^{0}}{\partial \gamma_{0}^{\prime}}\left\{\delta A^{r} \cos \left(k_{p}^{\prime} z^{\prime}\right)-\delta A^{i} \sin \left(k_{p}^{\prime} z^{\prime}\right)\right. \\
& -\int_{-\infty}^{t^{\prime}} d t^{\prime \prime} \exp \left[-i \Delta \omega^{\prime}\left(t^{\prime \prime}-t^{\prime}\right)\right] \\
& \left.\times\left[-i \Delta \omega^{\prime} \delta A^{r} \cos \left(k_{p}^{\prime} z^{\prime \prime}\right)+i \Delta \omega^{\prime} \delta A^{i} \sin \left(k_{p}^{\prime} z^{\prime \prime}\right)\right]\right\}
\end{aligned}
$$

Note that the argument $k_{p}^{\prime} z^{\prime}$ of $\delta F\left(p_{z}^{\prime}, k_{p}^{\prime} z^{\prime}, \Delta k^{\prime}, \Delta \omega^{\prime}\right)$ corresponds to a fast spatial variation, in contrast to the slow variation of $\exp \left(i \Delta k^{\prime} z^{\prime}\right)$. Substituting Eqs.(50) and (53) into Eq.(47) then gives

$$
\begin{aligned}
& 2 \omega_{s}^{\prime}\left[i\left(-\Delta \omega^{\prime}+v_{g}^{\prime} \Delta k^{\prime}\right) \delta A^{r}-v_{g}^{\prime} \epsilon k_{p}^{\prime} \delta A^{i}\right] \\
& \quad=\frac{4 \pi e^{2} a_{w}}{m} \frac{\hat{n}_{b}^{\prime}}{2 \pi} \int_{0}^{2 \pi} d\left(k_{p}^{\prime} z^{\prime}\right) \int_{-\infty}^{\infty} d p_{z}^{\prime} \delta F\left(p_{z}^{\prime}, k_{p}^{\prime} z^{\prime}, \Delta k^{\prime}, \Delta \omega^{\prime}\right) \frac{\sin \left(k_{p}^{\prime} z^{\prime}\right)}{\gamma^{\prime}\left(z^{\prime}, p_{z}^{\prime}\right)} \\
& 2 \omega_{s}^{\prime}\left[i\left(-\Delta \omega^{\prime}+v_{g}^{\prime} \Delta k^{\prime}\right) \delta A^{i}+v_{g}^{\prime} \epsilon k_{p}^{\prime} \delta A^{r}\right] \\
& \quad=\frac{4 \pi e^{2} a_{w}}{m} \frac{\hat{n}_{b}^{\prime}}{2 \pi} \int_{0}^{2 \pi} d\left(k_{p}^{\prime} z^{\prime}\right) \int_{-\infty}^{\infty} d p_{z}^{\prime} \delta F\left(p_{z}^{\prime}, k_{p}^{\prime} z^{\prime}, \Delta k^{\prime}, \Delta \omega^{\prime}\right) \frac{\cos \left(k_{p}^{\prime} z^{\prime}\right)}{\gamma^{\prime}\left(z^{\prime}, p_{z}^{\prime}\right)}
\end{aligned}
$$

In obtaining Eq.(55), use is made of the assumption that $\exp \left(i \Delta k^{\prime} z^{\prime}\right)$ varies slowly compared to $\sin \left(k_{p}^{\prime} z^{\prime}\right)$ and $\cos \left(k_{p}^{\prime} z^{\prime}\right)$, so that $\exp \left(i \Delta k^{\prime} z^{\prime}\right)$ can be taken outside of the integration over $z^{\prime}$.

For convenience, we introduce the following notation 


$$
\begin{aligned}
\alpha_{\nu} \equiv & \frac{a_{w}^{2}}{2} \frac{1}{2 \pi} \int_{-\pi}^{\pi} d\left(k_{p}^{\prime} z^{\prime}\right) \exp \left(i \nu k_{p}^{\prime} z^{\prime}\right) \int_{-\infty}^{\infty} d p_{z}^{\prime} \frac{1}{\gamma_{0}^{\prime 2}} \frac{\partial f^{0}}{\partial \gamma_{0}^{\prime}} \\
\chi_{\nu}^{ \pm}\left(\Delta \omega^{\prime}\right) \equiv & -\frac{1}{2} i \Delta \omega^{\prime} a_{w}^{2} \hat{\omega}_{p b}^{\prime 2} \frac{1}{2 \pi} \int_{-\pi}^{\pi} d\left(h_{p}^{\prime} z^{\prime}\right) \exp \left(i \nu k_{p}^{\prime} z^{\prime}\right) \int_{-\infty}^{\infty} \frac{d p_{z}^{\prime}}{\gamma_{0}^{\prime 2}} \frac{\partial f^{0}}{\partial \gamma_{0}^{\prime}} \\
& \times \int_{-\infty}^{t^{\prime}} d t^{\prime \prime} \exp \left\{-i\left[\Delta \omega^{\prime}\left(t^{\prime \prime}-t^{\prime}\right)\right] \pm i k_{p}^{\prime}\left[z^{\prime \prime}\left(t^{\prime \prime}\right)-z^{\prime}\right]\right\}
\end{aligned}
$$

where

$$
\hat{\omega}_{p b}^{\prime 2}=\frac{4 \pi \hat{n}_{b}^{\prime} e^{2}}{m}
$$

is the nonrelativistic plasma frequency-squared in the ponderomotive frame. Because $\int_{-\infty}^{\infty} d p_{z}^{\prime} \frac{1}{\gamma_{0}^{\prime 2}} \frac{\partial f^{0}}{\partial \gamma_{0}^{\prime}}$ is an even function of $z^{\prime}$, it is readily shown that

$$
\alpha_{\nu}=\alpha_{-\nu}^{*}=\alpha_{-\nu}=\alpha_{\nu}^{*}
$$

where * denotes complex conjugate. Substituting (54) into (55), and making use of (58), we obtain the following coupled equations relating the perturbation amplitudes $\delta A^{r}$ and $\delta A^{i}$

$$
\begin{aligned}
& \left\{2 i \omega_{s}^{\prime}\left(v_{g}^{\prime} \Delta k^{\prime}-\Delta \omega^{\prime}\right)-\frac{1}{2 i}\left[\chi_{2}^{+}\left(\Delta \omega^{\prime}\right)-\chi_{0}^{+}\left(\Delta \omega^{\prime}\right)+\chi_{0}^{-}\left(\Delta \omega^{\prime}\right)-\chi_{-2}^{-}\left(\Delta \omega^{\prime}\right)\right]\right\} \delta A^{r} \\
& =\left\{2 \omega_{s}^{\prime} v_{g}^{\prime} \epsilon k_{p}^{\prime}-\hat{\omega}_{p b}^{\prime 2}\left(\alpha_{2}-\alpha_{0}\right)+\frac{1}{2}\left\{\chi_{2}^{+}\left(\Delta \omega^{\prime}\right)-\chi_{0}^{+}\left(\Delta \omega^{\prime}\right)-\chi_{0}^{-}\left(\Delta \omega^{\prime}\right)+\chi_{-2}^{-}\left(\Delta \omega^{\prime}\right)\right]\right\} \delta A^{i}, \\
& \left\{2 i \omega_{s}^{\prime}\left(v_{g}^{\prime} \Delta k^{\prime}-\Delta \omega^{\prime}\right)+\frac{1}{2 i}\left[\chi_{2}^{+}\left(\Delta \omega^{\prime}\right)+\chi_{0}^{+}\left(\Delta \omega^{\prime}\right)-\chi_{0}^{-}\left(\Delta \omega^{\prime}\right)-\chi_{-2}^{-}\left(\Delta \omega^{\prime}\right)\right]\right\} \delta A^{i} \\
& =\left\{-2 \omega_{s}^{\prime} v_{g}^{\prime} \epsilon k_{p}^{\prime}-\hat{\omega}_{p b}^{\prime 2}\left(\alpha_{2}+\alpha_{0}\right)+\frac{1}{2}\left[\chi_{2}^{+}\left(\Delta \omega^{\prime}\right)+\chi_{0}^{+}\left(\Delta \omega^{\prime}\right)+\chi_{0}^{-}\left(\Delta \omega^{\prime}\right)+\chi_{-2}^{-}\left(\Delta \omega^{\prime}\right)\right]\right\} \delta A^{r} .
\end{aligned}
$$

Therefore, for $\delta A^{r}$ and $\delta A^{i}$ not to vanish, $\Delta k^{\prime}$ and $\Delta \omega^{\prime}$ must satisfy the dispersion relation

$$
\begin{aligned}
& {\left[\left(v_{g}^{\prime} \Delta k^{\prime}-\Delta \omega^{\prime}\right)+D_{0}^{-}\right]\left[\left(v_{g}^{\prime} \Delta k^{\prime}-\Delta \omega^{\prime}\right)-D_{1}^{+}\right]} \\
& \quad=\left[v_{g}^{\prime} \epsilon k_{p}^{\prime}-\frac{\hat{\omega}_{p b}^{\prime 2}}{2 \omega_{s}^{\prime}}\left(\alpha_{2}-\alpha_{0}\right)+D_{1}^{-}\right]\left[v_{g}^{\prime} \epsilon k_{p}^{\prime}+\frac{\hat{\omega}_{p b}^{\prime 2}}{2 \omega_{s}^{\prime}}\left(\alpha_{2}+\alpha_{0}\right)-D_{0}^{+}\right],
\end{aligned}
$$


where $D_{n}^{ \pm}$is defined by

$$
D_{n}^{ \pm}=\frac{1}{4 \omega_{s}^{\prime}}\left[\chi_{2}^{+}\left(\Delta \omega^{\prime}\right) \pm \chi_{0}^{+}\left(\Delta \omega^{\prime}\right)\right]+(-1)^{n} \frac{1}{4 \omega_{s}^{\prime}}\left[\chi_{0}^{-}\left(\Delta \omega^{\prime}\right) \pm \chi_{-2}^{-}\left(\Delta \omega^{\prime}\right)\right]
$$

for $n=0,1$, and $\chi_{\nu}^{ \pm}\left(\Delta \omega^{\prime}\right)$ and $\alpha_{\nu}$ are defined in (56). Furthermore, $\epsilon k_{p}^{\prime}$ and $v_{g}^{\prime}$ are defined in Eqs.(25) and (27). Equation (60) is the desired kinetic dispersion relation describing the linear stability properties for small-amplitude perturbations about the general equilibrium distribution function $f^{0}\left(\gamma_{0}^{\prime}\right)$ and the wave field $a_{s}^{0}\left(z^{\prime}\right)=$ $\hat{a}_{s}^{0} \exp \left[i \delta_{s}^{0}\left(z^{\prime}\right)\right]$.

Making use of the symmetry property of Eq.(40), it can be shown that

$$
\begin{aligned}
& z^{\prime \prime}\left(t^{\prime \prime},-z^{\prime},-p_{z}^{\prime}\right)=-z^{\prime \prime}\left(t^{\prime \prime}, z^{\prime}, p_{z}^{\prime}\right), \\
& p_{z}^{\prime \prime}\left(t^{\prime \prime},-z^{\prime},-p_{z}^{\prime}\right)=-p_{z}^{\prime \prime}\left(t^{\prime \prime}, z^{\prime}, p_{z}^{\prime}\right),
\end{aligned}
$$

where $z^{\prime \prime}\left(t^{\prime \prime}\right)$ and $p_{z}^{\prime \prime}\left(t^{\prime \prime}\right)$ are the orbits of the particle with "initial" conditions $z^{\prime \prime}\left(t^{\prime \prime}=\right.$ $\left.t^{\prime}\right)=z^{\prime}$ and $p_{z}^{\prime \prime}\left(t^{\prime \prime}=t^{\prime}\right)=p_{z}^{\prime}$. By changing the signs of the variables $z^{\prime}$ and $p_{z}^{\prime}$ in Eq.(56), and using Eq.(62), it is straightforward to show that

$$
\chi_{-\nu}^{-}\left(\Delta \omega^{\prime}\right)=\chi_{\nu}^{+}\left(\Delta \omega^{\prime}\right)
$$

for the cases where only trapped electrons are present in prescribing the equilibrium distribution $f^{0}\left(\gamma_{0}^{\prime}\right)$. Therefore, the kinetic dispersion relation $(60)$ reduces to

$$
\begin{aligned}
\left(v_{g}^{\prime} \Delta k^{\prime}-\Delta \omega^{\prime}\right)^{2} & =\left\{v_{g}^{\prime} \epsilon k_{p}^{\prime}-\frac{\hat{\omega}_{p b}^{\prime 2}}{2 \omega_{s}^{\prime}}\left(\alpha_{2}-\alpha_{0}\right)+\frac{1}{2 \omega_{s}^{\prime}}\left[\chi_{2}^{+}\left(\Delta \omega^{\prime}\right)-\chi_{0}^{+}\left(\Delta \omega^{\prime}\right)\right]\right\} \\
\times & \left\{v_{g}^{\prime} \epsilon k_{p}^{\prime}+\frac{\hat{\omega}_{p b}^{\prime 2}}{2 \omega_{s}^{\prime}}\left(\alpha_{2}+\alpha_{0}\right)-\frac{1}{2 \omega_{s}^{\prime}}\left[\chi_{2}^{+}\left(\Delta \omega^{\prime}\right)+\chi_{0}^{+}\left(\Delta \omega^{\prime}\right)\right]\right\},
\end{aligned}
$$

for distributions $f^{0}\left(\gamma_{0}^{\prime}\right)$ with only trapped electrons. Equation (64) is one of the main results of this paper and can be used to investigate detailed stability behavior for a wide range of trapped-electron distributions $f^{0}\left(\gamma_{0}^{\prime}\right)$. 


\section{Sideband Instability for Uniformly Populated Trapped- Electron Distribution}

As an application of the kinetic equilibrium and stability formalism developed in Secs. II and III, we now consider an equilibrium state in which the electromagnetic wave has constant amplitude, $\hat{a}_{s}^{0}=$ const., and the electrons are all trapped and uniformly populated in the ponderomotive potential, i.e.,

$$
f^{0}\left(\gamma_{0}^{\prime}\right)= \begin{cases}\frac{1}{N}, & \hat{\gamma}_{-}^{\prime}<\gamma_{0}^{\prime}<\hat{\gamma}_{+}^{\prime} \\ 0, & \text { otherwise }\end{cases}
$$

Here, $\hat{\gamma}_{+}^{\prime 2}=1+\left(a_{w}+\hat{a}_{s}^{0}\right)^{2}, \hat{\gamma}_{-}^{\prime 2}=1+\left(a_{w}-\hat{a}_{s}^{0}\right)^{2}$, and $N$ is the appropriate normalization constant. Moreover, $a_{w}$ and $\hat{a}_{s}^{0}$ are defined in Eqs.(6) and (22), respectively. The contours corresponding to $\gamma_{0}^{\prime}=$ const. in phase space are shown in Fig.1. For the distribution described by Eq.(65), electrons are uniformly populated in the phase-space region inside the separatrix. The normalization constant $N$ in Eq.(65) is determined from

$$
\frac{1}{2 \pi} \int_{-\pi}^{\pi} d\left(k_{p}^{\prime} z^{\prime}\right) \int_{-\infty}^{\infty} d p_{z}^{\prime} f^{0}\left(\gamma_{0}^{\prime}\right)=1
$$

where $\gamma_{0}^{\prime}$ is defined in Eq.(29). The integral over $p_{z}^{\prime}$ in Eq.(66) can be converted into an integral over $\gamma_{0}^{\prime}$. From Eq.(29), we obtain

$$
d p_{z}^{\prime}=m^{2} c^{2} \frac{\gamma_{0}^{\prime} d \gamma_{0}^{\prime}}{p_{z}^{\prime}}= \pm \frac{\gamma_{0}^{\prime} m c d \gamma_{0}^{\prime}}{\left[\gamma_{0}^{\prime 2}-1-a^{2}\left(z^{\prime}\right)\right]^{1 / 2}}
$$

where the plus sign corresponds to $p_{z}^{\prime}>0$ and the minus sign corresponds to $p_{z}^{\prime}<0$. Furthermore, $a^{2}\left(z^{\prime}\right)$ is defined by

$$
a^{2}\left(z^{\prime}\right)=a_{w}^{2}+\hat{a}_{s}^{0}-2 a_{w} \hat{a}_{s}^{0} \cos \left(k_{p}^{\prime} z^{\prime}\right)
$$

Making use of Eqs.(65), (66) and (67), it follows that

$$
N=\frac{1}{2 \pi} \int_{-\pi}^{\pi} d\left(k_{p}^{\prime} z^{\prime}\right) \int_{\gamma_{-}^{\prime}\left(z^{\prime}\right)}^{\hat{\gamma}_{+}^{\prime}} d \gamma_{0}^{\prime} \frac{2 \gamma_{0}^{\prime} m c}{\left[\gamma_{0}^{\prime 2}-1-a^{2}\left(z^{\prime}\right)\right]^{1 / 2}}
$$


where $\gamma_{-}^{\prime}\left(z^{\prime}\right)$ is defined by

$$
\gamma_{-}^{\prime}\left(z^{\prime}\right) \equiv\left[1+a^{2}\left(z^{\prime}\right)\right]^{1 / 2}
$$

Carrying out the integrals over $\gamma_{0}^{\prime}$ and $k_{p}^{\prime} z^{\prime}$ in Eq. (69) gives

$$
N=\frac{8 m c}{\pi}\left(a_{w} \hat{a}_{s}^{0}\right)^{1 / 2}
$$

Substituting Eq.(71) into Eq.(65), the equilibrium distribution function $f^{\circ}\left(\gamma_{0}^{\prime}\right)$ can be expressed as

$$
f^{0}\left(\gamma_{0}^{\prime}\right)=\left\{\begin{array}{cl}
\frac{\pi}{8 m c\left(a_{w} \hat{a}_{s}^{0}\right)^{1 / 2}}=\text { const., } & \hat{\gamma}_{-}^{\prime}<\gamma_{0}^{\prime}<\hat{\gamma}_{+}^{\prime}, \\
0, & \text { otherwise. }
\end{array}\right.
$$

The equilibrium phase shift of the electromagnetic wave, $\epsilon k_{p}^{\prime}$, is determined from Eq.(28). Substituting Eq.(72) into Eq.(28), and using Eq.(67) to convert the integral over $p_{z}^{\prime}$ into an integral over $\gamma_{0}^{\prime}$, the equilibrium phase shift $\epsilon k_{p}^{\prime}$ for a uniformly populated trapped-electron distribution is given by

$$
\epsilon k_{p}^{\prime}=\frac{\pi \hat{\omega}_{p b}^{\prime 2} a_{w}^{1 / 2}}{8 k_{s}^{\prime} c^{2}\left(\hat{a}_{s}^{0}\right)^{3 / 2}} \frac{1}{2 \pi} \int_{-\pi}^{\pi} d\left(k_{p}^{\prime} z^{\prime}\right) \int_{\hat{\gamma}_{-}^{\prime}\left(z^{\prime}\right)}^{\hat{\gamma}_{+}^{\prime}} d \gamma_{0}^{\prime} \frac{\cos \left(k_{p}^{\prime} z^{\prime}\right)}{\left[\gamma_{0}^{\prime 2}-1-a^{2}\left(z^{\prime}\right)\right]^{1 / 2}},
$$

where $\hat{\omega}_{p b}^{\prime 2}=4 \pi \hat{n}_{b}^{\prime} e^{2} / m$ is the nonrelativistic plasma frequency-squared in the ponderomotive frame. The integral over $\gamma_{0}^{\prime}$ can be carried out to give

$$
\begin{aligned}
\epsilon k_{p}^{\prime} & =\frac{\hat{\omega}_{p b}^{\prime 2} a_{w}^{1 / 2}}{32 k_{s}^{\prime} c^{2}\left(\hat{a}_{s}^{0}\right)^{3 / 2}} \int_{-\pi}^{\pi} d\left(k_{p}^{\prime} z^{\prime}\right) \cos \left(k_{p}^{\prime} z^{\prime}\right) \ln \left[\frac{\hat{\gamma}_{+}^{\prime}+\left(4 a_{w} \hat{a}_{s}^{0}\right)^{1 / 2} \cos \left(\frac{k_{p}^{\prime} z^{\prime}}{2}\right)}{\hat{\gamma}_{+}^{\prime}-\left(4 a_{w} \hat{a}_{s}^{0}\right)^{1 / 2} \cos \left(\frac{k_{p}^{\prime} z^{\prime}}{2}\right)}\right] \\
& =\frac{\dot{\omega}_{p b}^{\prime 2} a_{w}^{1 / 2}}{4 k_{s}^{\prime} c^{2}\left(\hat{a}_{s}^{0}\right)^{3 / 2}} W\left[\frac{\left(4 a_{w} \hat{a}_{s}^{0}\right)^{1 / 2}}{\hat{\gamma}_{+}^{\prime}}\right],
\end{aligned}
$$

where $W(x)$ is defined by

$$
W(x) \equiv \frac{1}{2} \int_{0}^{\frac{\pi}{2}} d \theta \cos (2 \theta) \ln \left(\frac{1+x \cos \theta}{1-x \cos \theta}\right) .
$$

For $|x| \ll 1$, which is the regime of practical interest, it can be shown that the power series expansion of $W(x)$ is

$$
\begin{aligned}
W(x) & =\sum_{k=1}^{\infty} \frac{C_{2 k-1}^{w}}{2 k+1} x^{2 k-1} \\
C_{m}^{w} & =\frac{(m-1)(m-3) \cdots 2}{m(m-1) \cdots 3}=\frac{2^{\frac{m-1}{2}} \cdot\left(\frac{m-1}{2}\right) !}{m ! !}
\end{aligned}
$$


for odd integer $m$. For example, the first three terms of $C_{m}^{w}$ are

$$
C_{1}^{w}=1 . \quad C_{3}^{w}=\frac{2}{3}, \quad C_{5}^{w}=\frac{8}{15}
$$

In the regime of practical interest, $a_{w}$ is of order unity and $\left|\hat{a}_{s}^{0}\right| \ll 1$. Therefore, it is a good approximation to replace $W\left[\left(4 a_{w} \hat{a}_{s}^{0}\right)^{1 / 2} / \hat{\gamma}_{+}^{\prime}\right]$ in Eq. $(74)$ by $\left(4 a_{w} \hat{a}_{s}^{0}\right)^{1 / 2} / 3 \hat{\gamma}_{+}^{\prime}$. The expression for $\epsilon k_{p}^{\prime}$ correct to the lowest order is then given by

$$
\epsilon k_{p}^{\prime}=\frac{\hat{\omega}_{p b}^{\prime 2} a_{w}}{6 k_{s}^{\prime} c^{2} \hat{a}_{s}^{0} \hat{\gamma}_{+}^{\prime}} .
$$

For future reference, we introduce the small dimensionless parameter $\Gamma_{0}^{\prime 3}$, and the bounce frequency $\hat{\omega}_{B}^{\prime}\left(\gamma_{0}^{\prime}\right)$ of a deeply trapped electron with energy $\gamma_{0}^{\prime}$ defined by

$$
\begin{aligned}
\Gamma_{0}^{\prime 3} & =\frac{a_{w}^{2} \hat{\omega}_{p b}^{\prime 2}}{4 \omega_{s}^{\prime} \hat{\gamma}^{\prime 3} c k_{p}^{\prime}}, \\
\hat{\omega}_{B}^{\prime 2}\left(\gamma_{0}^{\prime}\right) & =\frac{a_{w} \hat{a}_{s}^{0} c^{2} k_{p}^{\prime 2}}{\gamma_{0}^{\prime 2}} .
\end{aligned}
$$

In the definition of $\Gamma_{0}^{\prime 3}$, the characteristic energy $\hat{\gamma}^{\prime}$ of an electron trapped in the ponderomotive potential is given approximately by

$$
\hat{\gamma}^{\prime}=\left(1+a_{w}^{2}\right)^{1 / 2}
$$

[see Eq.(29) with $p_{z}^{\prime}=0$ and $\left.\hat{a}_{s}^{0} \ll a_{w}.\right]$ Making use of Eq.(78), and the approximation $\hat{\gamma}_{+}^{\prime} \simeq \hat{\gamma}^{\prime}$ (for $\hat{a}_{s}^{0} \ll a_{w}$ ), Eq.(77) can be expressed in the equivalent form

$$
v_{g}^{\prime} \in k_{p}^{\prime}=\frac{2}{3} \frac{\left(\Gamma_{0}^{\prime} c k_{p}^{\prime}\right)^{3}}{\hat{\omega}_{B}^{\prime 2}\left(\hat{\gamma}^{\prime}\right)},
$$

where $v_{g}^{\prime}$ is defined in Eq.(27). In Eq. $(78),\left(3^{1 / 2} / 2\right) \Gamma_{0}^{\prime} c k_{p}^{\prime}$ is the familiar small-signal gain (temporal growth rate) ${ }^{75}$ calculated in the ponderomotive frame.

\section{A. Particle Orbits at the Separatrix}

The kinetic dispersion relation (64) contains integrals over $t^{\prime \prime}$, which require a determination of the orbits $z^{\prime \prime}\left(t^{\prime \prime}\right)$ and $p_{z}^{\prime \prime}\left(t^{\prime \prime}\right)$ from the equations of the motion. For the 
distribution function given in Eq.(72), it follows that $\partial f^{0} / \partial \gamma_{0}^{\prime}=-(1 / N) \delta\left(\gamma_{0}^{\prime}-\hat{\gamma}_{+}^{\prime}\right)$. Therefore, to evaluate the necessary orbit integrals, the relevant orbits are those of electrons moving on the separatrix with $\gamma_{0}^{\prime}=\hat{\gamma}_{+}^{\prime}$. Setting $\gamma_{0}^{\prime}$ equal to $\hat{\gamma}_{+}^{\prime}$, Eq.(29) readily gives

$$
\left(\hat{\gamma}_{+}^{\prime} \frac{d z^{\prime \prime}}{d t^{\prime \prime}}\right)^{2}=c^{2}\left[\hat{\gamma}_{+}^{\prime 2}-1-a^{2}\left(z^{\prime \prime}\right)\right],
$$

where $\hat{\gamma}_{+}^{\prime 2} \equiv 1+\left(a_{w}+\hat{a}_{s}^{0}\right)^{2}$, and $a^{2}\left(z^{\prime \prime}\right)$ is given in Eq. $(68)$. The "initial" conditions of the orbits are

$$
\begin{aligned}
& z^{\prime \prime}\left(t^{\prime \prime}=t^{\prime}\right)=z^{\prime} \\
& {\left[\frac{d}{d t^{\prime \prime}} z^{\prime \prime}\left(t^{\prime \prime}\right)\right]_{t^{\prime \prime}=t^{\prime}}= \pm \frac{c}{\hat{\gamma}_{+}^{\prime}}\left[\hat{\gamma}_{+}^{2}-1-a^{2}\left(z^{\prime}\right)\right]^{1 / 2} .}
\end{aligned}
$$

The plus sign in Eq.(82) corresponds to electrons moving in the forward direction $\left(p_{z}^{\prime}>0\right)$, whereas the minus sign corresponds to electrons with $p_{z}^{\prime}<0$. Making use of Eq.(68) and the definition of $\hat{\gamma}_{+}^{\prime}$, Eq.(81) can be expressed in the form

$$
\begin{aligned}
& \frac{1}{2} \frac{d\left(k_{p}^{\prime} z^{\prime \prime}\right)}{d t^{\prime \prime}}= \pm \frac{1}{\tau_{B}} \cos \left(\frac{k_{p}^{\prime} z^{\prime \prime}}{2}\right), \\
& \frac{1}{\tau_{B}^{2}}=\hat{\omega}_{B}^{\prime 2}\left(\hat{\gamma}_{+}^{\prime}\right)=\frac{a_{w} \hat{a}_{s}^{0} c^{2} k_{p}^{\prime 2}}{\hat{\gamma}_{+}^{\prime 2}} .
\end{aligned}
$$

Again, the plus (minus) sign in Eq. (83) corresponds to forward-moving (backwardmoving) electrons in the ponderomotive frame. Because of the periodicity of the equilibrium configuration, without loss of generality, we concentrate on the ponderomotive potential in the region centered at $z^{\prime}=0$ and spanning the interval $-\pi<k_{p}^{\prime} z^{\prime}<\pi$. The solution to Eq.(83) subject to the "initial" conditions in Eq.(82) is given by

$$
\begin{aligned}
k_{p}^{\prime} z^{\prime \prime}\left(t^{\prime \prime}\right) & =-\pi+4 \tan ^{-1}\left[u \exp \left( \pm \frac{\tau^{\prime}}{\tau_{B}}\right)\right], \\
\tau^{\prime} & =t^{\prime \prime}-t^{\prime} \\
u & =\tan \left(\frac{k_{p}^{\prime} z^{\prime}+\pi}{4}\right) .
\end{aligned}
$$

For $t^{\prime \prime} \rightarrow \infty$, it follows from Eq.(84) that

$$
k_{p}^{\prime} z^{\prime \prime}\left(t^{\prime \prime} \rightarrow \infty\right)= \pm \pi
$$


which means that all the forward-moving (backward-moving) electrons approach $k_{p}^{\prime} z^{\prime}=\pi\left(k_{p}^{\prime} z^{\prime}=-\pi\right)$ as $t^{\prime \prime}$ approaches infinity.

\section{B. Evaluation of Dielectric Coefficients}

Converting the integral over $p_{z}^{\prime}$ in Eq.(56) to an integral over $\gamma_{0}^{\prime}$ by using Eq.(67) gives

$$
\begin{aligned}
\chi_{\nu}^{+}\left(\Delta \omega^{\prime}\right)= & -\frac{m c}{2} i \Delta \omega^{\prime} a_{w}^{2} \hat{\omega}_{p b}^{\prime 2} \frac{1}{2 \pi} \int_{-\pi}^{\pi} d\left(k_{p}^{\prime} z^{\prime}\right) \int_{\gamma_{-}^{\prime}\left(z^{\prime}\right)}^{\hat{\gamma}_{+}^{\prime}} \frac{d \gamma_{0}^{\prime}}{\gamma_{0}^{\prime}} \frac{\partial f^{0}}{\partial \gamma_{0}^{\prime}} \frac{\exp \left[i(\nu-1) k_{p}^{\prime} z^{\prime}\right]}{\left[\gamma_{0}^{\prime 2}-1-a\left(z^{\prime}\right)\right]^{1 / 2}} \\
& \times \frac{1}{2} \int_{-\infty}^{0} d \tau^{\prime} \exp \left(-i \Delta \omega^{\prime} \tau^{\prime}\right)\left\{\exp \left[i k_{p}^{\prime} z_{+}^{\prime \prime}\left(\tau^{\prime}\right)\right]+\exp \left[i k_{p}^{\prime} z_{-}^{\prime \prime}\left(\tau^{\prime}\right)\right]\right\}
\end{aligned}
$$

for distributions containing only trapped electrons. Here, $z_{ \pm}^{\prime}\left(\tau^{\prime}\right)$ is the trajectory of an electron with initial conditions $z^{\prime \prime}\left(\tau^{\prime}=0\right)=z^{\prime}$ and $p_{z}^{\prime \prime}\left(\tau^{\prime}=0\right)= \pm m c\left[\gamma_{0}^{\prime 2}-1-\right.$ $\left.a\left(z^{\prime}\right)\right]^{1 / 2}$. Furthermore, $a\left(z^{\prime}\right)$ and $\gamma_{-}^{\prime}\left(z^{\prime}\right)$ are defined in Eqs.(68) and (70), respectively. Interchanging the order of the $k_{p}^{\prime} z^{\prime}$ and $\gamma_{0}^{\prime}$ integrations, it follows that

$$
\int_{-\pi}^{\pi} d\left(k_{\mathrm{p}}^{\prime} z^{\prime}\right) \int_{\gamma_{-}^{\prime}\left(z^{\prime}\right)}^{\hat{\gamma}_{+}^{\prime}} d \gamma_{0}^{\prime} \cdots \rightarrow \int_{\hat{\gamma}_{-}^{\prime}}^{\dot{\gamma}_{+}^{\prime}} d \gamma_{0}^{\prime} \int_{-k_{p}^{\prime} z_{T}^{\prime}\left(\gamma_{0}^{\prime}\right)}^{k_{p}^{\prime} z_{T}^{\prime}\left(\gamma_{0}^{\prime}\right)} d\left(k_{p}^{\prime} z^{\prime}\right) \cdots
$$

for the trapped electrons. In Eq. (87), $\hat{\gamma}_{-}^{\prime} \equiv\left[1+\left(a_{w}-\hat{a}_{s}^{0}\right)^{2}\right]^{1 / 2}$ is the absolute minimum value of $\gamma_{-}^{\prime}\left(z^{\prime}\right)=\left[1+a^{2}\left(z^{\prime}\right)\right]^{1 / 2}$, which occurs for $k_{p}^{\prime} z^{\prime}=0$. Moreover, the turning points $\pm z_{T}^{\prime}\left(\gamma_{0}^{\prime}\right)$ for a trapped electron with energy $\gamma_{0}^{\prime}$ are determined from

$$
\gamma_{0}^{\prime 2}=1+a^{2}\left(z_{T}^{\prime}\right)=1+a_{w}^{2}+\left(\hat{a}_{s}^{0}\right)^{2}-2 a_{w} \hat{a}, \cos \left(k_{p}^{\prime} z_{T}^{\prime}\right)
$$

or equivalently,

$$
\gamma_{0}^{\prime 2}=\hat{\gamma}_{-}^{\prime 2}+4 a_{w} \hat{a}_{s}^{0} \sin ^{2}\left[k_{p}^{\prime} z_{T}^{\prime}\left(\gamma_{0}^{\prime}\right) / 2\right]
$$

Making use of Eq.(87) to interchange the order of the $k_{p}^{\prime} z^{\prime}$ and $\gamma_{0}^{\prime}$ integrations in Eq. (86) gives

$$
\begin{aligned}
\chi_{\nu}^{+}\left(\Delta \omega^{\prime}\right)= & -\frac{m c}{2} i \Delta \omega^{\prime} a_{w}^{2} \hat{\omega}_{p b}^{\prime 2} \int_{\hat{\gamma}_{-}^{\prime}}^{\hat{\gamma}_{+}^{\prime}} \frac{d \gamma_{0}^{\prime}}{\gamma_{0}^{\prime}} \frac{\partial f^{0}}{\partial \gamma_{0}^{\prime}} \int_{-k_{p}^{\prime} z_{T}^{\prime}\left(\gamma_{0}^{\prime}\right)}^{k_{p}^{\prime} z_{T}^{\prime}\left(\gamma_{0}^{\prime}\right)} \frac{d\left(k_{p}^{\prime} z^{\prime}\right)}{2 \pi} \frac{\exp \left[i(\nu-1) k_{p}^{\prime} z^{\prime}\right]}{\left[\gamma_{0}^{\prime 2}-1-a\left(z^{\prime}\right)\right]^{1 / 2}} \\
& \times \frac{1}{2} \int_{-\infty}^{0} d \tau^{\prime} \exp \left(-i \Delta \omega^{\prime} \tau^{\prime}\right)\left\{\exp \left[i k_{p}^{\prime} z_{+}^{\prime \prime}\left(\tau^{\prime}\right)\right]+\exp \left[i k_{p}^{\prime} z_{-}^{\prime \prime}\left(\tau^{\prime}\right)\right]\right\}
\end{aligned}
$$


for distribution functions containing only trapped electrons. Similarly, the first equation in (56) can be expressed in the equivalent form

$$
\begin{gathered}
\alpha_{\nu}=\frac{a_{w}^{2}}{2} \int_{\hat{\gamma}_{-}^{\prime}}^{\hat{\gamma}_{+}^{\prime}} \frac{m c d \gamma_{0}^{\prime}}{\gamma_{0}^{\prime}} \frac{\partial f^{0}}{\partial \gamma_{0}^{\prime}} \int_{-k_{p}^{\prime} z_{T}^{\prime}\left(\gamma_{0}^{\prime}\right)}^{k_{p}^{\prime} z_{T}^{\prime}\left(\gamma_{v^{\prime}}^{\prime}\right)} \frac{d\left(k_{p}^{\prime} z^{\prime}\right)}{2 \pi} \frac{\exp \left(i \nu k_{p}^{\prime} z^{\prime}\right)}{\left[\gamma_{0}^{\prime 2}-1-a\left(z^{\prime}\right)\right]^{1 / 2}} \\
=\frac{m c}{2} i \Delta \omega^{\prime} a_{w}^{2} \int_{\hat{\gamma}_{-}^{\prime}}^{\hat{\gamma}_{+}^{\prime}} \frac{d \gamma_{0}^{\prime}}{\gamma_{0}^{\prime}} \frac{\partial f^{0}}{\partial \gamma_{0}^{\prime}} \int_{-k_{p}^{\prime} z_{T}^{\prime}\left(\gamma_{0}^{\prime}\right)}^{k_{p}^{\prime} z_{T}^{\prime}\left(\gamma_{0}^{\prime}\right)} \frac{d\left(k_{p}^{\prime} z^{\prime}\right)}{2 \pi} \frac{\exp \left[i(\nu-1) k_{p}^{\prime} z^{\prime}\right]}{\left[\gamma_{0}^{\prime 2}-1-a\left(z^{\prime}\right)\right]^{1 / 2}} \\
\times \int_{-\infty}^{0} d \tau^{\prime} \exp \left(-i \Delta \omega^{\prime} \tau^{\prime}\right) \exp \left(i k_{p}^{\prime} z^{\prime}\right) .
\end{gathered}
$$

Combining Eqs.(90) and (91), we obtain

$$
\begin{aligned}
\hat{\omega}_{p b}^{\prime 2} \alpha_{\nu}-\chi_{\nu}^{+}\left(\Delta \omega^{\prime}\right)= & -\frac{m c}{4} i \Delta \omega^{\prime} a_{w}^{2} \hat{\omega}_{p b}^{\prime 2} \int_{\hat{\gamma}_{-}^{\prime}}^{\hat{\gamma}_{+}^{\prime}} \frac{d \gamma_{0}^{\prime}}{\gamma_{0}^{\prime}} \frac{\partial f^{0}}{\partial \gamma_{0}^{\prime}} \int_{-k_{p}^{\prime} z_{T}^{\prime}\left(\gamma_{0}^{\prime}\right)}^{k_{p}^{\prime} z_{T}^{\prime}\left(\gamma_{0}^{\prime}\right)} \frac{d\left(k_{p}^{\prime} z^{\prime}\right)}{2 \pi} \\
& \times \frac{\exp \left[i(\nu-1) k_{p}^{\prime} z^{\prime}\right]}{\left[\gamma_{0}^{\prime 2}-1-a\left(z^{\prime}\right)\right]^{1 / 2}} \int_{-\infty}^{0} d \tau^{\prime} \exp \left(-i \Delta \omega^{\prime} \tau^{\prime}\right) \\
& \times\left\{2 \exp \left(i k_{p}^{\prime} z^{\prime}\right)-\exp \left[i k_{p}^{\prime} z_{+}^{\prime \prime}\left(\tau^{\prime}\right)\right]-\exp \left[i k_{p}^{\prime} z_{-}^{\prime \prime}\left(\tau^{\prime}\right)\right]\right\}
\end{aligned}
$$

which is the required combination in the dispersion relation (64) for $\nu=0,2$.

Equation (92) is valid for any equilibrium distribution function which contains only trapped electrons. Differentiating Eq.(72) with respect to $\gamma_{0}^{\prime}$ gives

$$
\frac{\partial f^{0}}{\partial \gamma_{0}^{\prime}}=\frac{\pi}{4 m c\left(a_{w} \hat{a}_{s}^{0}\right)^{1 / 2}}\left[\delta\left(\gamma_{0}^{\prime}-\hat{\gamma}_{-}^{\prime}\right)-\delta\left(\gamma_{0}^{\prime}-\hat{\gamma}_{+}^{\prime}\right)\right],
$$

for a uniform distribution of trapped electrons. It is evident from Eqs.(92) and (93) that the relevant electron orbits are those either on the separatrix $\left(\gamma_{0}^{\prime}=\hat{\gamma}_{+}^{\prime}\right)$ or trapped at the bottom of the ponderomotive potential $\left(\gamma_{0}^{\prime}=\hat{\gamma}_{-}^{\prime}\right)$. The orbit of an electron with $\gamma_{0}^{\prime}=\hat{\gamma}_{-}^{\prime}$ is simply $z_{+}^{\prime \prime}\left(\tau^{\prime}\right)=z_{-}^{\prime \prime}\left(\tau^{\prime}\right)=z^{\prime}$, and it is evident from Eq.(92) that the corresponding contribution to the dielectric function $\hat{\omega}_{p b}^{\prime 2} \alpha_{\nu}-\chi_{\nu}^{+}\left(\Delta \omega^{\prime}\right)$ vanishes. Therefore, substituting Eq.(93) into Eq.(92), the dielectric function reduces to

$$
\begin{aligned}
\hat{\omega}_{p b}^{\prime 2} \alpha_{\nu}-\chi_{\nu}^{+}\left(\Delta \omega^{\prime}\right) & =\frac{i}{64} \frac{\Delta \omega^{\prime} a_{w} \hat{\omega}_{p b}^{\prime 2}}{\hat{\gamma}_{+}^{\prime} \hat{a}_{s}^{0}} \int_{-\infty}^{0} d \tau^{\prime} \exp \left(-i \Delta \omega^{\prime} \tau^{\prime}\right) \\
& \times \int_{-\pi}^{\pi} d\left(k_{p}^{\prime} z^{\prime}\right) \frac{\exp \left[i(\nu-1) k_{p}^{\prime} z^{\prime}\right]}{\cos \left(k_{p}^{\prime} z^{\prime} / 2\right)}\left\{2 \exp \left(i k_{p}^{\prime} z^{\prime}\right)\right. \\
& \left.-\exp \left[i k_{p}^{\prime} z_{+}^{\prime \prime}\left(\tau^{\prime}\right)\right]-\exp \left[i k_{p}^{\prime} z_{-}^{\prime \prime}\left(\tau^{\prime}\right)\right]\right\}
\end{aligned}
$$


where $k_{p}^{\prime} z_{ \pm}^{\prime \prime}\left(\tau^{\prime}\right)$ is given by Eq.(84). In deriving Eq.(94), use has been made of Eq.(68) to express $\left[\hat{\gamma}_{+}^{\prime 2}-1-a^{2}\left(z^{\prime}\right)\right]^{1 / 2}$ as

$$
\left[\hat{\gamma}_{+}^{\prime 2}-1-a^{2}\left(z^{\prime}\right)\right]^{1 / 2}=\left(a_{w} \hat{a}_{s}^{0}\right)^{1 / 2}\left[2+2 \cos \left(k_{p}^{\prime} z^{\prime}\right)\right]^{1 / 2}=2\left(a_{w} \hat{a}_{s}^{0}\right)^{1 / 2} \cos \left(k_{p}^{\prime} z^{\prime} / 2\right)
$$

Moreover, the order of the $k_{p}^{\prime} z^{\prime}$ and $\tau^{\prime}$ integrations has been reversed.

Making use of Eq.(84), it can be shown after some algebraic manipulation (Appendix A) that

$$
\begin{aligned}
\int_{-\pi}^{\pi} d\left(k_{p}^{\prime} z^{\prime}\right) \frac{1}{\cos \left(k_{p}^{\prime} z^{\prime} / 2\right)}\left\{1-\exp \left[i k_{p}^{\prime} z_{ \pm}^{\prime \prime}\left(\tau^{\prime}\right)-i k_{p}^{\prime} z^{\prime}\right]\right\} \\
=\frac{32(\Gamma-1) \Gamma}{(\Gamma+1)^{3}} \ln \Gamma+\frac{16(\Gamma-1)^{2}}{(\Gamma+1)^{2}} \pm i \frac{32(\Gamma-1) \Gamma \pi}{(\Gamma+1)^{3}}
\end{aligned}
$$

where

$$
\Gamma=\exp \left(-\frac{\tau^{\prime}}{\tau_{B}}\right)
$$

Substituting Eq.(96) into Eq.(94) for $\nu=0$ gives

$$
\begin{aligned}
\hat{\omega}_{p b}^{\prime 2} \alpha_{0}-\chi_{0}^{+}\left(\Delta \omega^{\prime}\right) & =\frac{i}{2} \frac{\Delta \omega^{\prime} a_{w} \hat{\omega}_{p b}^{\prime 2}}{\hat{\gamma}_{+}^{\prime} \hat{a}_{s}^{0}} \int_{-\infty}^{0} d \tau^{\prime} \exp \left(-i \Delta \omega^{\prime} \tau^{\prime}\right) \\
\times & {\left[\frac{2 \Gamma(\Gamma-1)}{(\Gamma+1)^{3}} \ln \Gamma+\frac{(\Gamma-1)^{2}}{(\Gamma+1)^{2}}\right] . }
\end{aligned}
$$

Similarly, it can be shown from Eq.(84) that (Appendix A)

$$
\begin{gathered}
\int_{-\pi}^{\pi} d\left(k_{p}^{\prime} z^{\prime}\right) \frac{\exp \left(i k_{p}^{\prime} z^{\prime}\right)}{\cos \left(k_{p}^{\prime} z^{\prime} / 2\right)}\left\{\exp \left(i k_{p}^{\prime} z^{\prime}\right)-\exp \left[i k_{p}^{\prime} z_{+}^{\prime \prime}\left(\tau^{\prime}\right)\right]\right\} \\
=16\left[\frac{\Gamma^{2}+10 \Gamma+1}{3(\Gamma-1)^{2}}-\frac{2 \Gamma(\Gamma+1)}{(\Gamma-1)^{3}} \ln \Gamma\right] .
\end{gathered}
$$

Substituting Eq.(99) into Eq.(94) for $\nu=2$ gives

$$
\begin{gathered}
\hat{\omega}_{p b}^{\prime 2} \alpha_{2}-\chi_{2}^{+}\left(\Delta \omega^{\prime}\right)=\frac{i}{2} \frac{\Delta \omega^{\prime} a_{w} \hat{\omega}_{p b}^{\prime 2}}{\hat{\gamma}_{+}^{\prime} \hat{a}_{s}^{0}} \int_{-\infty}^{0} d \tau^{\prime} \exp \left(-i \Delta \omega^{\prime} \tau^{\prime}\right) \\
\times\left[\frac{\Gamma^{2}+10 \Gamma+1}{3(\Gamma-1)^{2}}-\frac{2 \Gamma(\Gamma+1)}{(\Gamma-1)^{3}} \ln \Gamma\right] .
\end{gathered}
$$


After further algebraic manipulation (Appendix B), the dielectric functions in Eqs. (98) and (100) can be expressed as

$$
\begin{aligned}
& \hat{\omega}_{p b}^{\prime 2} \alpha_{0}-\chi_{0}^{+}=-\frac{a_{w} \hat{\omega}_{p b}^{\prime 2}}{2 \hat{\gamma}_{+}^{\prime} \hat{a}_{s}^{0}}\left\{1+i \alpha\left[1-2 i \alpha^{2} \frac{d}{d \alpha} Y(\alpha)\right]\right\}, \\
& \hat{\omega}_{p b}^{\prime 2} \alpha_{2}-\chi_{2}^{+}=-\frac{a_{w} \hat{\omega}_{p b}^{\prime 2}}{2 \hat{\gamma}_{+}^{\prime} \hat{a}_{s}^{0}}\left\{\frac{1}{3}-i \alpha\left[2 \alpha^{2} Z(\alpha)-(2 i \alpha+1)\right]\right\} .
\end{aligned}
$$

Here, $\alpha=\Delta \omega^{\prime} \tau_{B}$, and the functions $Y(\alpha)$ and $Z(\alpha)$ are defined by

$$
\begin{aligned}
& Y(\alpha)= \begin{cases}\int_{0}^{\infty} d x \frac{\exp (i \alpha x)}{\exp (x)+1}, & \operatorname{Im} \alpha>-1, \\
\int_{0}^{\infty} d x \frac{\exp (-i \alpha x)}{\exp (x)+1}+\frac{i}{\alpha}-\frac{\pi i}{\sinh (\pi \alpha)}, & \operatorname{Im} \alpha \leq-1,\end{cases} \\
& Z(\alpha)= \begin{cases}\int_{0}^{\infty} d x \frac{x \exp (i \alpha x)}{\exp (x)-1}, & \operatorname{Im} \alpha>-1, \\
-\int_{0}^{\infty} d x \frac{x \exp (-i \alpha x)}{\exp (x)-1}+\frac{1}{\alpha^{2}}-\frac{\pi^{2}}{\sinh ^{2}(\pi \alpha)}, & \operatorname{Im} \alpha \leq-1 .\end{cases}
\end{aligned}
$$

Evidently, properties of the functions $Y(\alpha)$ and $Z(\alpha)$ are key in describing detailed stability behavior.

\section{Maximum Growth Rate in the Weak-Pump Regime}

Substituting Eqs.(101) and (102) into the dispersion relation Eq.(64) and making use of Eqs.(27), (80) and (83), the dispersion relation can be expressed as

$$
\begin{aligned}
\left(\Delta K^{\prime}\right. & \left.-\Delta \Omega^{\prime}\right)^{2}=\frac{1}{P}\left(\Delta \Omega^{\prime}\right)^{2}\left[1+\Delta \Omega^{\prime} \frac{d}{d\left(\Delta \Omega^{\prime}\right)} Y\left(\Delta \Omega^{\prime}\right)+i \Delta \Omega^{\prime} Z\left(\Delta \Omega^{\prime}\right)\right] \\
& \times\left[\frac{1}{3}+i \Delta \Omega^{\prime}-\left(\Delta \Omega^{\prime}\right)^{2}+\left(\Delta \Omega^{\prime}\right)^{3} \frac{d}{d\left(\Delta \Omega^{\prime}\right)} Y\left(\Delta \Omega^{\prime}\right)-i\left(\Delta \Omega^{\prime}\right)^{3} Z\left(\Delta \Omega^{\prime}\right)\right]
\end{aligned}
$$

for a uniform distribution of trapped electrons extending from the bottom of the

ponderomotive potential to the separatrix at $\gamma_{0}^{\prime}=\dot{\gamma}_{+}^{\prime}$. Here, $\Delta K^{\prime}$ and $\Delta \Omega^{\prime}$ are the normalized wavenumber and frequency defined by

$$
\Delta K^{\prime}=v_{g}^{\prime} \Delta k^{\prime} \tau_{B}, \quad \Delta \Omega^{\prime}=\Delta \omega^{\prime} \tau_{B}
$$


Moreover, the dimensionless parameter $P$, which is a measure of the pump strength, is defined by

$$
P=\frac{1}{9\left(v_{g}^{\prime} \epsilon k_{p}^{\prime} \tau_{B}\right)^{2}}=\frac{1}{4}\left[\frac{\hat{\omega}_{B}^{\prime}\left(\hat{\gamma}^{\prime}\right)}{\Gamma_{0}^{\prime} c k_{p}^{\prime}}\right]^{6} .
$$

In obtaining Eq.(105), use has been made of Eqs.(80) and (83), and the approximation $\dot{\gamma}_{+}^{\prime} \simeq \hat{\gamma}^{\prime}\left(\right.$ for $\left.\hat{a}_{s}^{0} \ll a_{w}\right)$.

Equation(103) can be solved numerically for the real oscillation frequency $\operatorname{Re}\left(\Delta \Omega^{\prime}\right)$ and the growth rate $\operatorname{Im}\left(\Delta \Omega^{\prime}\right)$ in terms of $\Delta K^{\prime}, \epsilon k_{p}^{\prime}$ and $\tau_{B}$. For a uniform population of trapped electrons it is evident from Eq.(103) that properties of the sideband instability depend on the average electron density $\left(\hat{\omega}_{p b}^{\prime 2}\right)$ and the amplitudes of the wiggler field $\left(a_{w}\right)$ and the equilibrium wave field $\left(\hat{a}_{s}^{0}\right)$ only through the combination occurring in the dimensionless pump parameter $P$. The numerical results for different values of the pump strength $P$ will be presented in Sec.VI and compared with those for a general step-function distribution of trapped electrons which does not extend to the separatrix $\gamma_{0}^{\prime}=\hat{\gamma}_{+}^{\prime}$.

Although the growth rate $\operatorname{Im}\left(\Delta \Omega^{\prime}\right)$ must generally be determined numerically from Eq.(103), analytical estimates of the maximum growth rate can be made in the weak-pump $\operatorname{limit}(P \ll 1)$. It is evident from Eq.(105) that this limit corresponds to the regime with high electron density (large $\hat{\omega}_{p b}^{\prime 2}$ ) and small wave amplitude $\hat{a}_{s}^{0}$. However, it should be kept in mind that $\epsilon k_{p}^{\prime} \ll k_{p}^{\prime}$ is assumed in the present analysis. Therefore, the limiting case where $\hat{a}_{s}^{0}$ approaches zero at finite value of the electron density is excluded from the present model.

For $|\alpha| \gg 1$ and $\operatorname{Im}(\alpha)>-1$, the functions $Y(\alpha)$ and $Z(\alpha)$ can be expanded in an asymptotic series in powers of $1 / \alpha$ as

$$
\begin{aligned}
-i \frac{d}{d \alpha} Y(\alpha) & =\frac{1}{2}\left(\frac{i}{\alpha}\right)^{2}-\frac{1}{2}\left(\frac{i}{\alpha}\right)^{3}+\frac{1}{2}\left(\frac{i}{\alpha}\right)^{5}-\frac{3}{2}\left(\frac{i}{\alpha}\right)^{7}+\frac{17}{2}\left(\frac{i}{\alpha}\right)^{9} \cdots \\
Z(\alpha) & =\left(\frac{i}{\alpha}\right)-\frac{1}{2}\left(\frac{i}{\alpha}\right)^{2}+\frac{1}{6}\left(\frac{i}{\alpha}\right)^{3}-\frac{1}{30}\left(\frac{i}{\alpha}\right)^{5}+\frac{1}{42}\left(\frac{i}{\alpha}\right)^{7}-\frac{1}{30}\left(\frac{i}{\alpha}\right)^{9} \cdots
\end{aligned}
$$


Substituting Eq.(106) into Eq.(103) gives the approximate dispersion relation

$$
\left(\Delta K^{\prime}-\Delta \Omega^{\prime}\right)^{2}=\frac{1}{9 P}\left[1+\frac{3}{\left(\Delta \Omega^{\prime}\right)^{2}}\right]
$$

for $|\alpha|=\left|\Delta \Omega^{\prime}\right| \gg 1$ and $\operatorname{Im}\left(\Delta \Omega^{\prime}\right)>-1$. Close examination of Eq.(107) (Appendix C) shows that the maximum growth rate occurs at

$$
\left(\Delta K^{\prime}\right)_{M} \simeq \frac{1}{3 P^{1 / 2}}
$$

Moreover, the maximum normalized growth rate and the corresponding normalized real frequency are given by

$$
\begin{aligned}
\operatorname{Im}\left(\Delta \Omega^{\prime}\right)_{M} & =\frac{3^{1 / 2}}{2^{4 / 3}} \frac{1}{P^{1 / 6}} \\
\operatorname{Re}\left(\Delta \Omega^{\prime}\right)_{M} & =\frac{1}{2^{4 / 3}} \frac{1}{P^{1 / 6}}
\end{aligned}
$$

Therefore, in the weak-pump limit, the maximum growth rate for a uniformly populated trapped-electron distribution is given by

$$
\operatorname{Im}\left(\Delta \omega^{\prime}\right)_{M}=\frac{3^{1 / 2}}{2^{4 / 3}} \frac{1}{P^{1 / 3} \tau_{B}}=\frac{\sqrt{3}}{2}\left(\Gamma_{0}^{\prime} c k_{p}^{\prime}\right)=\frac{3^{1 / 2}}{2}\left(\frac{\hat{\omega}_{p b}^{\prime 2} a_{w}^{2} c^{2} k_{p}^{\prime 2}}{4 \dot{\gamma}^{\prime 3} \omega_{s}^{\prime}}\right)^{1 / 3} .
$$

It is noteworthy that, in the weak-pump limit, the maximum growth rate is independent of the equilibrium amplitude of the signal wave. If solved in the ponderomotive frame and in the weak-pump limit $(P \ll 1)$, the dispersion relation obtained by Davidson and Wurtele ${ }^{24}$ for deeply trapped electrons gives the same maximum growth rate as Eq.(110). 


\section{Dispersion Relation for General Trapped-Electron Dis- tribution}

In this section, we remove the assumption that the distribution of trapped electrons is uniform, and simplify the dispersion relation (64) for general distribution function $f^{0}\left(\gamma_{0}^{\prime}\right)$

\section{A. Trapped-Electron Orbits $\left(\hat{\gamma}_{-}^{\prime}<\gamma_{0}^{\prime}<\hat{\gamma}_{+}^{\prime}\right)$}

For trapped electrons with energy $\gamma_{0}^{\prime}$, Eq.(29) readily gives

$$
\left(\gamma_{0}^{\prime} \frac{d z^{\prime \prime}}{d t^{\prime \prime}}\right)^{2}=c^{2}\left[\gamma_{0}^{\prime 2}-1-a^{2}\left(z^{\prime \prime}\right)\right]
$$

where $1+\left(a_{w}+\hat{a}_{s}^{0}\right)^{2} \equiv \hat{\gamma}_{+}^{\prime 2}>\gamma_{0}^{\prime 2}>\hat{\gamma}_{-}^{\prime 2} \equiv 1+\left(a_{w}-\hat{a}_{s}^{0}\right)^{2}$, and $a\left(z^{\prime \prime}\right)$ is defined in Eq.(68). The "initial" conditions for the orbits are

$$
\begin{aligned}
z^{\prime \prime}\left(t^{\prime \prime}=t^{\prime}\right) & =z^{\prime} \\
\frac{d}{d t^{\prime \prime}} z^{\prime \prime}\left(t^{\prime \prime}=t^{\prime}\right) & = \pm \frac{c}{\gamma_{0}^{\prime}}\left[\gamma_{0}^{\prime 2}-1-a^{2}\left(z^{\prime}\right)\right]^{1 / 2}
\end{aligned}
$$

As before, the plus (minus) sign in Eq.(112) corresponds to electrons moving forward (backward) in the ponderomotive frame. We define

$$
\kappa_{T}^{2}=\frac{\gamma_{0}^{\prime 2}-\hat{\gamma}_{+}^{\prime 2}+4 a_{w} \hat{a}_{s}^{0}}{4 a_{w} \hat{a}_{s}^{0}}
$$

and introduce the new independent variable $\eta^{\prime \prime}$ defined by

$$
\kappa_{T} \sin \eta^{\prime \prime}=\sin \left(k_{p}^{\prime} z^{\prime \prime} / 2\right)
$$

Equation (111) can then be expressed as

$$
\left(\frac{d \eta^{\prime \prime}}{d t^{\prime \prime}}\right)^{2}=\hat{\omega}_{B}^{\prime 2}\left(\gamma_{0}^{\prime}\right)\left(1-\kappa_{T}^{2} \sin ^{2} \eta^{\prime \prime}\right)
$$

where the bounce frequency $\hat{\omega}_{B}^{\prime}\left(\gamma_{0}^{\prime}\right)$ near the bottom of the ponderomotive potential is defined in Eq. (78) 
Equation (115) can be solved for $\eta^{\prime \prime}\left(t^{\prime \prime}\right)$ in terms of elliptic integrals, and then the orbit $z^{\prime \prime}\left(t^{\prime \prime}\right)$ calculated from Eq.(114). ${ }^{21}$ Without presenting algebraic details, we obtain the trapped electron trajectory ${ }^{81}$

$$
\sin \left(k_{p}^{\prime} z^{\prime \prime} / 2\right)=\kappa_{T} \sin \eta^{\prime \prime}=\kappa_{T} \operatorname{sn}\left[\hat{\omega}_{B}^{\prime}\left(\gamma_{0}^{\prime}\right)\left(t^{\prime \prime}-t^{\prime}\right)+\frac{2}{\pi} K\left(\kappa_{T}\right) \theta_{0}\right],
$$

where $\theta_{0}$ is related to the initial condition $z^{\prime}=z^{\prime \prime}\left(t^{\prime \prime}=t^{\prime}\right)$ by

$$
\sin \left(k_{p}^{\prime} z^{\prime} / 2\right)=\kappa_{T} \operatorname{sn}\left[\frac{2}{\pi} K\left(\kappa_{T}\right) \theta_{0}\right] .
$$

The range of $\theta_{0}$ is chosen such that

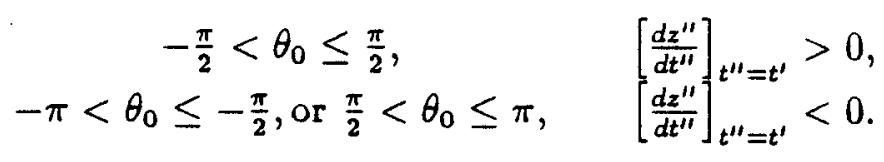

In Eqs.(116) and (117), the functions $K(u)$ and $\operatorname{sn}(u)$ are the complete elliptic integral of the first kind and the sine-amplitude of the Jacobian elliptic function, respectively. ${ }^{81}$ The function $\operatorname{sn}(u)$ is periodic with period $4 K\left(\kappa_{T}\right)$. Therefore, the trapped electron motion given in Eq.(116) has bounce frequency

$$
\omega_{b}^{\prime}\left(\gamma_{0}^{\prime}\right)=2 \pi f_{b}=\frac{\pi \hat{\omega}_{B}^{\prime}\left(\gamma_{0}^{\prime}\right)}{2 K^{\prime}\left(\kappa_{T}\right)} .
$$

Equation (116) can also be expressed in harmonic representation as $^{21}$

$$
z^{\prime \prime}\left(t^{\prime \prime}\right)=z^{\prime}+\sum_{n=1}^{\infty} z_{n}^{T}\left\{\sin (2 n-1)\left[\omega_{b}^{\prime}\left(\gamma_{0}^{\prime}\right)\left(t^{\prime \prime}-t^{\prime}\right)+\theta_{0}\right]-\sin (2 n-1) \theta_{0}\right\}
$$

where the coefficient $z_{n}^{T}$ is defined by

$$
\begin{aligned}
& z_{n}^{T}=\frac{8}{k_{p}^{\prime}} \frac{1}{(2 n-1)} \frac{a_{T}^{n-1 / 2}}{1+a_{n}^{2 n-1}}, \\
& a_{T}=\exp \left\{-\pi \frac{K\left[\left(1-\kappa_{T}^{2}\right)^{1 / 2}\right]}{K\left(\kappa_{T}\right)}\right\} .
\end{aligned}
$$

Taking the limit $\kappa_{T} \ll 1$, which corresponds to the orbit of a deeply trapped electron, Eq.(119) reduces to the motion of a simple harmonic oscillator with bounce frequency $\hat{\omega}_{B}^{\prime}\left(\gamma_{0}^{\prime}\right)$. For general value of trapped electron energy $\gamma_{0}^{\prime}$, it is evident from Eqs.(118) and (120) that the bounce frequency $\omega_{b}^{\prime}\left(\gamma_{0}^{\prime}\right)$ decreases as $\gamma_{0}^{\prime}$ is increased, and the higher harmonics in Eq.(119) play a significant role. 


\section{B. Evaluation of Dielectric Coefficients}

In this section, use is made of the trapped-electron trajectories [Eqs.(116) and (117)] to carry out the integrals over $t^{\prime \prime}$ and $k_{p}^{\prime} z^{\prime}$ in Eq.(56). Making use of Eqs.(67), (87) and (117), the following conversion of integration variables is obtained for the trapped electrons

$$
\int_{-\pi}^{\pi} d\left(k_{p}^{\prime} z^{\prime}\right) \int d p_{z}^{\prime} \cdots \rightarrow \int_{\hat{\gamma}_{-}^{\prime}}^{\hat{\gamma}_{+}^{\prime}} d \gamma_{0}^{\prime} \int_{-\pi}^{\pi} d \theta_{0}\left|\frac{d\left(k_{p}^{\prime} z^{\prime}\right)}{d \theta_{0}}\right| \frac{\gamma_{0}^{\prime} m c}{\left[\gamma_{0}^{\prime 2}-1-a^{2}\left(z^{\prime}\right)\right]^{1 / 2}} \cdots
$$

Here, $a^{2}\left(z^{\prime}\right)$ is defined in Eq.(68). By differentiating both sides of Eq.(117), it can be shown that

$$
\begin{aligned}
&\left|\frac{d\left(k_{p}^{\prime} z^{\prime}\right)}{d \theta_{0}}\right|=\frac{4 K\left(\kappa_{T}\right)}{\pi\left(4 a_{w} \hat{a}_{s}^{0}\right)^{1 / 2}}\left[\gamma_{0}^{\prime 2}-1-a^{2}\left(z^{\prime}\right)\right]^{1 / 2}, \\
& \int_{\hat{\gamma}_{-}^{\prime}}^{\hat{\gamma}_{+}^{\prime}} d \gamma_{0}^{\prime} \int_{-\pi}^{\pi} d \theta_{0}\left|\frac{d\left(k_{p}^{\prime} z^{\prime}\right)}{d \theta_{0}}\right| \frac{\gamma_{0}^{\prime} m c}{\left[\gamma_{0}^{\prime 2}-1-a^{2}\left(z^{\prime}\right)\right]^{1 / 2}} \cdots \\
&=\int_{\hat{\gamma}_{-}^{\prime}}^{\hat{\gamma}_{+}^{\prime}} d \gamma_{0}^{\prime} \int_{-\pi}^{\pi} d \theta_{0} \frac{m c^{2} k_{p}^{\prime}}{\omega_{b}^{\prime}\left(\gamma_{0}^{\prime}\right)} \cdots,
\end{aligned}
$$

where $\omega_{b}^{\prime}\left(\gamma_{0}^{\prime}\right)$ is defined in Eq.(118). It readily follows from Eqs.(121) and (122) that the quantities $\alpha_{\nu}$ and $\chi_{\nu}^{+}\left(\Delta \omega^{\prime}\right)$ given in Eq.(56) can be expressed as

$$
\begin{aligned}
\alpha_{\nu}= & \frac{a_{w}^{2}}{2} \frac{m c^{2} k_{p}^{\prime}}{2 \pi} \int_{\hat{\gamma}_{-}^{\prime}}^{\hat{\gamma}_{+}^{\prime}} \frac{d \gamma_{0}^{\prime}}{\gamma_{0}^{\prime 2} \omega_{b}^{\prime}\left(\gamma_{0}^{\prime}\right)} \frac{\partial f^{0}}{\partial \gamma_{0}^{\prime}} \int_{-\pi}^{\pi} d \theta_{0} \exp \left(i \nu k_{p}^{\prime} z^{\prime}\right), \\
\chi_{\nu}^{+}\left(\Delta \omega^{\prime}\right)= & -\frac{1}{2} i \Delta \omega^{\prime} a_{w}^{2} \hat{\omega}_{p b}^{\prime 2} \frac{m c^{2} k_{p}^{\prime}}{2 \pi} \int_{\dot{\gamma}_{-}^{\prime}}^{\hat{\gamma}_{+}^{\prime}} \frac{d \gamma_{0}^{\prime}}{\gamma_{0}^{\prime 2} \omega_{b}^{\prime}\left(\gamma_{0}^{\prime}\right)} \frac{\partial f^{0}}{\partial \gamma_{0}^{\prime}} \int_{-\pi}^{\pi} d \theta_{0} \exp \left(i \nu k_{p}^{\prime} z^{\prime}\right) \\
& \times \int_{-\infty}^{t^{\prime}} d t^{\prime \prime} \exp \left\{-i\left[\Delta \omega^{\prime}\left(t^{\prime \prime}-t^{\prime}\right)\right]+i k_{p}^{\prime}\left[z^{\prime \prime}\left(t^{\prime \prime}\right)-z^{\prime}\right]\right\} .
\end{aligned}
$$

Making use of the Cauchy integral formula ${ }^{83}$ it can be shown from Eq.(117) that

$$
\begin{aligned}
& \cos \left(k_{p}^{\prime} z^{\prime}\right)=2 \frac{E\left(\kappa_{T}\right)}{K\left(\kappa_{T}\right)}-1+\sum_{l= \pm 2, \pm 4, \ldots} \frac{l \pi^{2}}{K^{2}\left(\kappa_{T}\right)} \frac{a_{T}^{l / 2}}{1-a_{T}^{l}} \exp \left(i l \theta_{0}\right), \\
& \sin \left(k_{p}^{\prime} z^{\prime}\right)=(-i) \sum_{l= \pm 1, \pm 3, \ldots} \frac{l \pi^{2}}{K^{2}\left(\kappa_{T}\right)} \frac{a_{T}^{l / 2}}{1+a_{T}^{l}} \exp \left(i l \theta_{0}\right)
\end{aligned}
$$

where $a_{T}$ is defined in Eq.(120). Moreover, $K(k)$ and $E(k)$ are the complete elliptic integrals of the first and second kind, respectively. In obtaining Eq.(124), use is made 
of the following series expansions of the Jacobian elliptic functions ${ }^{82}$

$$
\begin{array}{r}
\operatorname{sn}[u+i K(k)]=\frac{1}{k u}+\frac{1+k^{2}}{6 k} u+O\left(u^{3}\right) \\
\operatorname{dn}[u+i K(k)]=-\frac{i}{u}+\frac{2-k^{2}}{6} i u+O\left(u^{3}\right)
\end{array}
$$

to evaluate the residues. Here, sn and $\mathrm{dn}$ are Jacobian elliptic functions. ${ }^{81}$ Squaring the two equations in (124), adding them together and then operating on the sum with $\int_{-\pi}^{\pi} d \theta_{0}$ readily gives the identity

$$
1=\left[2 \frac{E\left(\kappa_{T}\right)}{K\left(\kappa_{T}\right)}-1\right]^{2}+\sum_{l= \pm 2, \pm 4, \cdots} \frac{l^{2} \pi^{4}}{K^{4}\left(\kappa_{T}\right)} \frac{a_{T}^{l}}{\left(1-a_{T}^{l}\right)^{2}}+\sum_{l= \pm 1, \pm 3, \ldots} \frac{l^{2} \pi^{4}}{K^{4}\left(\kappa_{T}\right)} \frac{a_{T}^{l}}{\left(1+a_{T}^{l}\right)^{2}}
$$

Substituting Eq.(124) into the first equation in (123) for $\nu=0$ and $\nu=2$, we obtain

$$
\begin{aligned}
\alpha_{0}= & \frac{a_{w}^{2} m c^{2} k_{p}^{\prime}}{2} \int_{\hat{\gamma}_{-}^{\prime}}^{\hat{\gamma}_{+}^{\prime}} \frac{d \gamma_{0}^{\prime}}{\gamma_{0}^{\prime 2} \omega_{b}^{\prime}\left(\gamma_{0}^{\prime}\right)} \frac{\partial f^{0}}{\partial \gamma_{0}^{\prime}}, \\
\alpha_{2}= & \frac{a_{w}^{2} m c^{2} k_{p}^{\prime}}{2} \int_{\hat{\gamma}_{-}^{\prime}}^{\hat{\gamma}_{+}^{\prime}} \frac{d \gamma_{0}^{\prime}}{\gamma_{0}^{\prime 2} \omega_{b}^{\prime}\left(\gamma_{0}^{\prime}\right)} \frac{\partial f^{0}}{\partial \gamma_{0}^{\prime}}\left\{\left[2 \frac{E\left(\kappa_{T}\right)}{K\left(\kappa_{T}\right)}-1\right]^{2}\right. \\
& \left.+\sum_{l= \pm 2, \pm 4, \cdots} \frac{l^{2} \pi^{4}}{K^{4}\left(\kappa_{T}\right)} \frac{a_{T}^{l}}{\left(1-a_{T}^{l}\right)^{2}}-\sum_{l= \pm 1, \pm 3, \ldots} \frac{l^{2} \pi^{4}}{K^{4}\left(\kappa_{T}\right)} \frac{a_{T}^{l}}{\left(1+a_{T}^{l}\right)^{2}}\right\} .
\end{aligned}
$$

Following the procedure used in deriving Eq.(124), it can be shown from Eq.(116) that

$$
\begin{aligned}
\cos \left(k_{p}^{\prime} z^{\prime \prime}\right) & =2 \frac{E\left(\kappa_{T}\right)}{K\left(\kappa_{T}\right)}-1+\sum_{l= \pm 2, \pm 4, \cdots} \frac{l \pi^{2}}{K^{2}\left(\kappa_{T}\right)} \frac{a_{T}^{l / 2}}{1-a_{T}^{l}} \exp \left\{i l\left[\omega_{b}^{\prime}\left(\gamma_{0}^{\prime}\right)\left(t^{\prime \prime}-t^{\prime}\right)+\theta_{0}\right]\right\} \\
\sin \left(k_{p}^{\prime} z^{\prime \prime}\right) & =(-i) \sum_{l= \pm 1, \pm 3, \cdots} \frac{l \pi^{2}}{K^{2}\left(\kappa_{T}\right)} \frac{a_{T}^{l / 2}}{1+a_{T}^{l}} \exp \left\{i l\left[\omega_{b}^{\prime}\left(\gamma_{0}^{\prime}\right)\left(t^{\prime \prime}-t^{\prime}\right)+\theta_{0}\right]\right\}
\end{aligned}
$$

where $z^{\prime \prime}$ is the electron trajectory in Eq.(116), and the bounce frequency $\omega_{b}^{\prime}\left(\gamma_{0}^{\prime}\right)$ is defined in Eq.(118). Substituting Eq.(128) into the second equation in (123) for $\nu=0$ and $\nu=2$, and carrying out the integrals over $t^{\prime \prime}$ and $\theta_{0}$ give the desired results 


$$
\begin{aligned}
& \chi_{0}^{+}\left(\Delta \omega^{\prime}\right)=\frac{1}{2} a_{w}^{2} \hat{\omega}_{p b}^{\prime 2} m c^{2} k_{p}^{\prime} \int_{\hat{\nu}_{-}^{\prime}}^{\dot{\nu}_{-}^{\prime}} \underset{\gamma_{0}^{\prime 2} \omega_{b}^{\prime \prime}\left(\gamma_{0}^{\prime}\right)}{d \gamma_{0}^{\prime}} \partial f^{0}\left\{\left[2 \frac{E\left(\kappa_{T}^{\prime}\right)}{K\left(\kappa_{T}\right)}-1\right]^{2}\right. \\
& +\sum_{l= \pm 2, \pm 4, \cdots} \frac{l^{2} \pi^{4}}{K^{4}\left(\kappa_{T}\right)} \frac{a_{T}^{l}}{\left(1-a_{T}^{l}\right)^{2}} \cdot \frac{\Delta \omega^{\prime}}{\Delta \omega^{\prime}-l \omega_{b}^{\prime}\left(\gamma_{0}^{\prime}\right)} \\
& \left.+\sum_{l= \pm 1, \pm 3, \cdots} \frac{l^{2} \pi^{4}}{K^{4}\left(\kappa_{T}\right)} \frac{a_{T}^{l}}{\left(1+a_{T}^{l}\right)^{2}} \frac{\Delta \omega^{\prime}}{\Delta \omega^{\prime}-l \omega_{b}^{\prime}\left(\gamma_{0}^{\prime}\right)}\right\}, \\
& \chi_{2}^{+}\left(\Delta \omega^{\prime}\right)=\frac{1}{2} a_{w}^{2} \hat{\omega}_{p b}^{\prime 2} m c^{2} k_{p}^{\prime} \int_{\hat{\gamma}_{-}^{\prime}}^{\hat{\gamma}_{+}^{\prime}} \frac{d \gamma_{0}^{\prime}}{\gamma_{0}^{\prime 2} \omega_{b}^{\prime}\left(\gamma_{0}^{\prime}\right)} \frac{\partial f^{0}}{\partial \gamma_{0}^{\prime}}\left\{\left[2 \frac{E\left(\kappa_{T}\right)}{K\left(\kappa_{T}\right)}-1\right]^{2}\right. \\
& +\sum_{l= \pm 2, \pm 4, \cdots} \frac{l^{2} \pi^{4}}{K^{4}\left(\kappa_{T}\right)} \frac{a_{T}^{l}}{\left(1-a_{T}^{l}\right)^{2}} \frac{\Delta \omega^{\prime}}{\Delta \omega^{\prime}-l \omega_{b}^{\prime}\left(\gamma_{0}^{\prime}\right)} \\
& \left.-\sum_{l= \pm 1, \pm 3, \ldots} \frac{l^{2} \pi^{4}}{K^{4}\left(\kappa_{T}\right)} \frac{a_{T}^{l}}{\left(1+a_{T}^{l}\right)^{2}} \frac{\Delta \omega^{\prime}}{\Delta \omega^{\prime}-l \omega_{b}^{\prime}\left(\gamma_{0}^{\prime}\right)}\right\} \text {. }
\end{aligned}
$$

Substituting Eqs.(127) and (129) into the kinetic dispersion relation (64) and making use of the identity in (126), we obtain

$$
\begin{aligned}
\left(v_{g}^{\prime} \Delta k^{\prime}-\Delta \omega^{\prime}\right)^{2}= & \left\{v_{g}^{\prime} \epsilon k_{p}^{\prime}\right. \\
- & \frac{a_{w}^{2} \hat{\omega}_{p b}^{\prime 2} m c^{2} k_{p}^{\prime}}{\omega_{s}^{\prime}} \sum_{l=2,4, \ldots} \int_{\hat{\gamma}_{-}^{\prime}}^{\hat{\gamma}_{+}^{\prime}} \frac{d \gamma_{0}^{\prime}}{\gamma_{0}^{\prime 2} \omega_{b}^{\prime}\left(\gamma_{0}^{\prime}\right)} \frac{\partial f^{0}}{\partial \gamma_{0}^{\prime}} \\
\times & \left.\frac{l^{4} \pi^{4}}{K^{4}\left(\kappa_{T}\right)} \frac{a_{T}^{l}}{\left(1-a_{T}^{l}\right)^{2}} \frac{\omega_{b}^{\prime 2}\left(\gamma_{0}^{\prime}\right)}{\left[\left(\Delta \omega^{\prime}\right)^{2}-l^{2} \omega_{b}^{\prime 2}\left(\gamma_{0}^{\prime}\right)\right]}\right\} \\
\times\left\{v_{g}^{\prime} \epsilon k_{p}^{\prime}\right. & -\frac{a_{w}^{2} \hat{\omega}_{p b}^{\prime 2} m c^{2} k_{p}^{\prime}}{\omega_{s}^{\prime}} \sum_{l=1,3, \cdots} \int_{\hat{\gamma}_{-}^{\prime}}^{\gamma_{+}^{\prime}} \frac{d \gamma_{0}^{\prime}}{\gamma_{0}^{\prime 2} \omega_{b}^{\prime}\left(\gamma_{0}^{\prime}\right)} \frac{\partial f^{0}}{\partial \gamma_{0}^{\prime}} \\
& \left.\times \frac{l^{4} \pi^{4}}{K^{4}\left(\kappa_{T}\right)} \frac{a_{T}^{l}}{\left(1+a_{T}^{l}\right)^{2}} \frac{\omega_{b}^{\prime 2}\left(\gamma_{0}^{\prime}\right)}{\left.\left(\Delta \omega^{\prime}\right)^{2}-l^{2} \omega_{b}^{\prime 2}\left(\gamma_{0}^{\prime}\right)\right]}\right\},
\end{aligned}
$$

where $\epsilon k_{p}^{\prime}$ is defined in Eq.(28). Making use of Eqs.(121), (122) and (124), the equilibrium phase shift $\epsilon k_{p}^{\prime}$ can be expressed as

$$
\epsilon k_{p}^{\prime}=\frac{a_{w} \hat{\omega}_{p b}^{\prime 2} m k_{p}^{\prime}}{2 k_{s}^{\prime} \hat{a}_{s}^{0}} \int_{\hat{\gamma}_{-}^{\prime}}^{\hat{\gamma}_{+}^{\prime}} \frac{d \gamma_{0}^{\prime}}{\gamma_{0}^{\prime} \omega_{b}^{\prime}\left(\gamma_{0}^{\prime}\right)}\left[2 \frac{E\left(\kappa_{T}\right)}{K\left(\kappa_{T}\right)}-1\right] f^{0}\left(\gamma_{0}^{\prime}\right) .
$$

Equation (130), which is one of the main results of this paper, is the desired form of the kinetic dispersion relation. Note that Equation (130) is valid for general distribution function $f^{0}\left(\gamma_{0}^{\prime}\right)$ consisting only of trapped electrons $\left(\hat{\gamma}_{-}^{\prime} \leq \gamma_{0}^{\prime}<\hat{\gamma}_{+}^{\prime}\right)$. 


\section{Sideband Instability for Step-Function Trapped-Electron Distribution}

\section{A. Dispersion Relation}

To study the influence of the distribution function on the sideband instability, we consider the particular form of trapped-electron distribution specified by (Fig.2)

$$
f^{0}\left(\gamma_{0}^{\prime}\right)= \begin{cases}\frac{1}{N\left(\gamma_{M}^{\prime}\right)}, & \hat{\gamma}_{-}^{\prime}<\gamma_{0}^{\prime}<\gamma_{M}^{\prime} \\ 0, & \text { otherwise. }\end{cases}
$$

Here, $\gamma_{M}^{\prime} \leq \hat{\gamma}_{+}^{\prime}$ is a parameter characterizing the maximum electron energy and $N\left(\gamma_{M}^{\prime}\right)$ is the normalization constant determined from

$$
1=\frac{1}{2 \pi} \int_{-\pi}^{\pi} d\left(k_{p}^{\prime} z^{\prime}\right) \int_{-\infty}^{\infty} d p_{z}^{\prime} f^{0}\left(\gamma_{0}^{\prime}\right)=m c^{2} k_{p}^{\prime} \int_{\hat{\gamma}_{-}^{\prime}}^{\hat{\gamma}_{+}^{\prime}} \frac{d \gamma_{0}^{\prime}}{\omega_{b}^{\prime}\left(\gamma_{0}^{\prime}\right)} f^{0}\left(\gamma_{0}^{\prime}\right)
$$

In obtaining Eq.(133), use has been made of Eqs.(121) and (122) to convert the integration variables from $z^{\prime}$ and $p_{z}^{\prime}$ to $\gamma_{0}^{\prime}$. Making use of Eqs.(118) and (133), the normalization constant $N\left(\gamma_{M}^{\prime}\right)$ is found to be

$$
N\left(\gamma_{M}^{\prime}\right)=\frac{8 m c}{\pi}\left(a_{w} \hat{a}_{s}^{0}\right)^{1 / 2}\left[\left(\kappa_{M I}^{2}-1\right) K\left(\kappa_{M}\right)+E\left(\kappa_{M}\right)\right]
$$

where

$$
\kappa_{M}^{2}=\frac{\gamma_{M}^{\prime 2}-\hat{\gamma}_{+}^{\prime 2}+4 a_{w} \hat{a}_{s}^{0}}{4 a_{w} \hat{a}_{s}^{0}}=\frac{\gamma_{M}^{\prime 2}-\hat{\gamma}_{-}^{\prime 2}}{4 a_{w} \hat{a}_{s}^{0}}
$$

The equilibrium phase shift $\epsilon k_{p}^{\prime}$ for the choice of distribution function in Eq.(132) is determined from Eqs.(131), (132) and (134). This gives

$$
v_{g}^{\prime} \epsilon k_{p}^{\prime}=v_{g}^{\prime} \epsilon_{0} k_{p}^{\prime} G_{M}\left(\gamma_{M}^{\prime}\right)
$$

where

$$
\begin{aligned}
v_{g}^{\prime} \epsilon_{0} k_{p}^{\prime} & \equiv \frac{2\left(\Gamma_{0}^{\prime} c k_{p}^{\prime}\right)^{3}}{\hat{\omega}_{B}^{\prime 2}\left(\hat{\gamma}^{\prime}\right)} \\
G_{M}\left(\gamma_{M I}^{\prime}\right) & \equiv \frac{1}{3}\left[\frac{2 \kappa_{M I}^{2} E\left(\kappa_{M I}\right)}{\left(\kappa_{M}^{2}-1\right) K\left(\kappa_{M I}\right)+E\left(\kappa_{M}\right)}-1\right] .
\end{aligned}
$$


Here, $\Gamma_{0}^{\prime}$ and $\hat{\omega}_{B}^{\prime 2}\left(\hat{\gamma}^{\prime}\right)$ are defined in Eq.(78). In obtaining Eq.(136), use has been made of the assumption $a_{w} \gg \hat{a}_{s}^{0}$ to replace $1 / \gamma_{0}^{\prime}$ by $1 / \hat{\gamma}^{\prime}=1 /\left(1+a_{w}^{2}\right)^{1 / 2}$ and bring it outside the integral over $\gamma_{0}^{\prime}$ in Eq.(131). From Eq.(132) it follows that

$$
\frac{\partial f^{0}}{\partial \gamma_{0}^{\prime}}=\frac{1}{N\left(\gamma_{M}^{\prime}\right)}\left[\delta\left(\gamma_{0}^{\prime}-\hat{\gamma}_{-}^{\prime}\right)-\delta\left(\gamma_{0}^{\prime}-\gamma_{M}^{\prime}\right)\right]
$$

where $N\left(\gamma_{M}^{\prime}\right)$ is defined in Eq.(134).

The kinetic dispersion relation (130) then reduces to

$$
\begin{aligned}
\left(v_{g}^{\prime} \Delta k^{\prime}-\Delta \omega^{\prime}\right)^{2}= & \left(v_{g}^{\prime} \epsilon_{0} k_{p}^{\prime}\right)^{2}\left\{G_{M}\left(\gamma_{M}^{\prime}\right)+\frac{K\left(\kappa_{M}\right) / 2}{\left(\kappa_{M}^{2}-1\right) K\left(\kappa_{M}\right)+E\left(\kappa_{M}\right)}\right. \\
& \left.\times \sum_{l=2,4, \cdots} \frac{l^{4} \pi^{4}}{K^{4}\left(\kappa_{M}\right)} \frac{a_{T M}^{l}}{\left(1-a_{T M}^{l}\right)^{2}} \frac{\omega_{b}^{\prime 2}\left(\gamma_{M}^{\prime}\right)}{\left(\Delta \omega^{\prime}\right)^{2}-l^{2} \omega_{b}^{\prime 2}\left(\gamma_{M}^{\prime}\right)}\right\} \\
\times & \left\{G_{M}\left(\gamma_{M}^{\prime}\right)+\frac{K\left(\kappa_{M}\right) / 2}{\left(\kappa_{M}^{2}-1\right) K\left(\kappa_{M}\right)+E\left(\kappa_{M}\right)}\right. \\
& \left.\times \sum_{l=1,3, \cdots} \frac{l^{4} \pi^{4}}{K^{4}\left(\kappa_{M}\right)} \frac{a_{T M}^{l}}{\left(1+a_{T M}^{l}\right)^{2}} \frac{\omega_{b}^{\prime 2}\left(\gamma_{M}^{\prime}\right)}{\left(\Delta \omega^{\prime}\right)^{2}-l^{2} \omega_{b}^{\prime 2}\left(\gamma_{M}^{\prime}\right)}\right\},
\end{aligned}
$$

where

$$
a_{T M}=\exp \left\{-\pi \frac{K\left[\left(1-\kappa_{M}^{2}\right)^{1 / 2}\right]}{K\left(\kappa_{M}\right)}\right\}
$$

and $v_{g}^{\prime}-k_{s}^{\prime} c^{2} / \omega_{s}^{\prime}$. In terms of dimensionless variables $\Delta \Omega^{\prime} \equiv \Delta \omega^{\prime} / \hat{\omega}_{B}^{\prime}\left(\gamma_{M}^{\prime}\right)$ and $\Delta K^{\prime} \equiv$ $v_{g}^{\prime} \Delta k^{\prime} / \hat{\omega}_{B}^{\prime}\left(\gamma_{M}^{\prime}\right)$, the dispersion relation Fq.(139) can be expressed in the equivalent form

$$
\begin{aligned}
\left(\Delta K^{\prime} \cdots \Delta \Omega^{\prime}\right)^{2}= & \frac{1}{P}\left\{G_{M}\left(\gamma_{M}^{\prime}\right)+\sum_{l=2,4, \cdots} \frac{Q_{l}^{-}\left(\kappa_{M}\right)}{\left[\frac{2 K\left(\kappa_{M}\right)}{\pi} \Delta \Omega^{\prime}\right]^{2}-l^{2}}\right\} \\
& \times\left\{G_{M}\left(\gamma_{M}^{\prime}\right)+\sum_{l=1,3, \cdots\left[\frac{2 K\left(\kappa_{M}\right)}{\pi} \Delta \Omega^{\prime}\right]^{2}-l^{2}} \frac{Q_{l}^{+}\left(\kappa_{M}\right)}{}\right.
\end{aligned}
$$

Here, the quantities $P$ and $Q_{l}^{ \pm}\left(\kappa_{M}\right)$ are defined by

$$
\begin{aligned}
P & =\frac{\hat{\omega}_{B}^{\prime 2}\left(\gamma_{M}^{\prime}\right)}{v_{g}^{\prime 2}\left(\epsilon_{0} k_{p}^{\prime}\right)^{2}} \simeq \frac{1}{4}\left[\frac{\hat{\omega}_{B}^{\prime}\left(\dot{\gamma}^{\prime}\right)}{\Gamma_{0}^{\prime} c k_{p}^{\prime}}\right]^{6}, \\
Q_{l}^{ \pm}\left(\kappa_{M}\right) & =\frac{K\left(\kappa_{M}\right) / 2}{\left(\kappa_{M}^{2}-1\right) K\left(\kappa_{M}\right)+E\left(\kappa_{M}\right)} \frac{l^{4} \pi^{4}}{K^{4}\left(\kappa_{M}\right)} \frac{a_{T M}^{l}}{\left(1 \pm a_{T M}^{l}\right)^{2}} .
\end{aligned}
$$


Note that the dimensionless pump strength $P$ defined in Eq.(142) is identical to that defined in Eq.(105) for a uniform distribution of trapped electrons extending to the separatrix $\gamma_{0}^{\prime}=\hat{\gamma}_{+}^{\prime}$.

\section{B. Maximum Growth Rate in the Weak-Pump Regime}

The dispersion relation (141) can be used to determine detailed properties of the

sideband instability for the choice of distribution function in Eq.(132). Although the dispersion relation (141) generally must be solved numerically, analytical estimates of the maximum growth rate can be made in the weak-pump regime.

First of all, a useful identity can be derived from Eq.(124). It is readily shown from Eq.(124) that

$$
\begin{aligned}
& \sum_{l=1,3, \cdots} Q_{l}^{+}\left(\kappa_{M}\right)+\sum_{l=2,4, \cdots} Q_{l}^{-}\left(\kappa_{M}\right) \\
& =\frac{K\left(\kappa_{M}\right) / 2}{E\left(\kappa_{M}\right)+\left(\kappa_{M}^{2}-1\right) K\left(\kappa_{M}\right)} \frac{1}{2} \int_{0}^{2 \pi} \frac{d \theta_{0}}{2 \pi}\left\{\left[\frac{d}{d \theta_{0}} \cos \left(k_{p}^{\prime} z^{\prime}\right)\right]^{2}+\left[\frac{d}{d \theta_{0}} \sin \left(k_{p}^{\prime} z^{\prime}\right)\right]^{2}\right\} \\
& =\frac{K\left(\kappa_{M}\right) / 2}{E\left(\kappa_{M}\right)+\left(\kappa_{M}^{2}-1\right) K\left(\kappa_{M}\right)} \frac{1}{2} \int_{0}^{2 \pi} \frac{d \theta_{0}}{2 \pi}\left|\frac{d\left(k_{p}^{\prime} z^{\prime}\right)}{d \theta_{0}}\right|_{\kappa_{T}=\kappa_{M}}^{2} .
\end{aligned}
$$

After some straightforward algebra, it follows from Eqs.(117) and (143) that

$$
\sum_{l=1,3, \cdots} Q_{l}^{+}\left(\kappa_{M}\right)+\sum_{l=2,4, \cdots} Q_{l}^{-}\left(\kappa_{M}\right)=\left[\frac{2 K\left(\kappa_{M}\right)}{\pi}\right]^{2}
$$

where $Q_{l}^{ \pm}\left(\kappa_{M}\right)$ is defined in Eq.(142).

In the weak-pump regime ( $P \ll 1$ ), the dispersion relation Eq. $(141)$ can be approximated near the maximum growth rate by (Appendix D)

$$
\begin{aligned}
\left(\Delta K^{\prime}-\Delta \Omega^{\prime}\right)^{2}= & \frac{1}{P}\left\{G_{M}\left(\gamma_{M}^{\prime}\right)+\left[\frac{\pi}{2 K\left(\kappa_{M}\right) \Delta \Omega^{\prime}}\right]^{2} \sum_{l=2,4, \cdots} Q_{l}^{-}\left(\kappa_{M}\right)\right\} \\
& \times\left\{G_{M}\left(\gamma_{M}^{\prime}\right)+\left[\frac{\pi}{2 K\left(\kappa_{M}\right) \Delta \Omega^{\prime}}\right]^{2} \sum_{l=1,3, \cdots} Q_{l}^{+}\left(\kappa_{M}\right)\right\} .
\end{aligned}
$$


Close examination of Eq.(145)(Appendix D) shows that the maximum growth occurs for

$$
\left(\Delta K^{\prime}\right)_{M} \simeq \frac{G_{M M}\left(\gamma_{M}^{\prime}\right)}{P^{1 / 2}},
$$

and the corresponding growth rate and real frequency shift are

$$
\begin{aligned}
\operatorname{Im}\left(\Delta \Omega^{\prime}\right)_{M} & =\frac{\sqrt{3}}{2^{4 / 3} P^{1 / 8}} \\
\operatorname{Re}\left(\Delta \Omega^{\prime}\right)_{M} & =\frac{1}{2^{4 / 3} P^{1 / 8}}
\end{aligned}
$$

Several points are noteworthy with regard to the stability properties calculated above in the weak-pump regime. These can be summarized as follows:

(a) The maximum growth rate and the corresponding real frequency shift Eq.(147) do not depend on $\gamma_{M}^{\prime}$, although $\left(\Delta K^{\prime}\right)_{M}$ does [Eq.(146)].

(b) Since the uniformly-populated trapped-electron distribution in Eq.(65) is a limiting case $\left(\gamma_{M}^{\prime} \rightarrow \hat{\gamma}_{+}^{\prime}\right)$ of the general step-function distribution in Eq.(132), it is not surprising to find that the maximum growth rate and the corresponding frequency shift in Eq.(147) are identical to those given in Eq.(109).

(c) The real frequency shift at maximum growth is given by

$$
\operatorname{Re}\left(\Delta \omega^{\prime}\right)_{M}=\frac{\hat{\omega}_{B}^{\prime}\left(\gamma_{M}^{\prime}\right)}{2^{4 / 3} P^{1 / 6}} \gg \omega_{b}^{\prime}\left(\gamma_{M}^{\prime}\right)
$$

in the weak-pump regime $(P \ll 1)$. By contrast, it will be evident from the numerical results presented in Sec.VI C that

$$
\operatorname{Re}\left(\Delta \omega^{\prime}\right)_{M} \simeq \omega_{b}^{\prime}\left(\gamma_{M}^{\prime}\right)
$$

in the strong-pump regime $(P \gg 1)$. Indeed, the synchrotron oscillations of the trapped electrons constitute the basic mechanism driving the sideband instability.

(d) The maximum growth rate in the weak-pump regime $(P \ll 1)$ is given by

$$
\operatorname{Im}\left(\Delta \omega^{\prime}\right)_{M}=\frac{\sqrt{3}}{2} \Gamma_{0}^{\prime} c k_{p}^{\prime}=\frac{\sqrt{3}}{2}\left(\frac{\hat{\omega}_{p b}^{\prime 2} a_{w}^{2} c^{2} k_{p}^{\prime 2}}{4 \hat{\gamma}^{\prime 3} \omega_{s}^{\prime}}\right)^{1 / 3},
$$

which is independent of $\hat{a}_{s}^{0}$, the equilibrium amplitude of the signal wave. 


\section{Numerical Results}

The dispersion relation (141) has been solved numerically for different values of pump strength $P$. In this regard, the effects of system parameters such as the beam density and the amplitudes of the wiggler field and the signal wave on stability behavior are investigated. On the other hand, by varying $\gamma_{M}^{\prime}$ for a fixed value of $P$, we are also able to examine the dependence of stability properties on the degree to which the ponderomotive potential is populated by the trapped electrons. For $\gamma_{M}^{\prime}$ very close to $\hat{\gamma}_{-}^{\prime}$, the distribution function (132) corresponds to deeply trapped electrons. In this limit $\left(\gamma_{M}^{\prime} \simeq \hat{\gamma}_{-}^{\prime}\right.$ and $\left.\kappa_{M} \simeq 0\right)$, the equilibrium phase shift [Eq.(136)] reduces to

$$
\epsilon k_{p}^{\prime}=\frac{2\left(\Gamma_{0}^{\prime} c k_{p}^{\prime}\right)^{3}}{\hat{\omega}_{B}^{\prime 2}\left(\hat{\gamma}^{\prime}\right)}=\frac{\hat{\omega}_{p b}^{\prime 2} a_{w}}{2 k_{s}^{\prime} c^{2} \hat{a}_{s}^{0} \hat{\gamma}^{\prime}},
$$

and the dispersion relation (141) reduces to

$$
\left(\Delta K^{\prime}-\Delta \Omega^{\prime}\right)^{2}=\frac{1}{P} \frac{\left(\Delta \Omega^{\prime}\right)^{2}}{\left(\Delta \Omega^{\prime}\right)^{2}-1} .
$$

Equations (151) and (152) agfee with the results obtained by Davidson and Wurtele. ${ }^{24}$

In the limit of a fully populated ponderomotive potential where $\gamma_{M}^{\prime}$ is very close to $\hat{\gamma}_{+}^{\prime}\left(\gamma_{M}^{\prime} \simeq \hat{\gamma}_{+}^{\prime}\right.$ and $\left.\kappa_{M} \simeq 1\right)$, the equilibrium phase shift [Eq.(136)] reduces to

$$
\epsilon k_{p}^{\prime}=\frac{2}{3} \frac{\left(\Gamma_{0}^{\prime} c k_{p}^{\prime}\right)^{3}}{\hat{\omega}_{B}^{\prime 2}\left(\hat{\gamma}^{\prime}\right)}=\frac{\dot{\omega}_{p b}^{\prime 2} a_{w}}{6 k_{s}^{\prime} c^{2} \hat{a}_{s}^{0} \gamma_{M}^{\prime}},
$$

which is identical to the result for a uniformly populated trapped-electron distribution given in Eq.(80). In this limit, however, the dispersion relation (141) is not well defined. Therefore, in calculating the growth rate and real frequency shift for $\kappa_{M}=1$, use will be made of Eq.(103). The complete dispersion relation (141) [or Eq.(103) for $\kappa_{M}=1$ ] has been solved numerically for three different values of pump strength $\left(P=10^{3}, P=1.0\right.$ and $\left.P=10^{-3}\right)$ and six different values of $\kappa_{M}$ to determine the

complex oscillation frequency $\Delta \Omega^{\prime}$ as a function of real wavenumber $\Delta K^{\prime}$. The results 
are shown in Figs.3-8. Although multiple roots are found for a given value of $\Delta K^{\prime \prime}$, only the unstable root with $\operatorname{Im}\left(\Delta \Omega^{\prime}\right)>0$ is presented in the figures.

Shown in Figs. 3 and 4 are the normalized growth rate $\operatorname{Im}\left(\Delta \Omega^{\prime}\right)=\operatorname{Im}\left(\Delta \omega^{\prime}\right) / \hat{\omega}_{B}^{\prime}\left(\gamma_{M}^{\prime}\right)$ and the corresponding real frequency shift $\operatorname{Re}\left(\Delta \Omega^{\prime}\right)=\operatorname{Re}\left(\Delta \omega^{\prime}\right) / \hat{\omega}_{B}^{\prime}\left(\gamma_{M}^{\prime}\right)$, respectively, for the choice of pump strength $P=10^{3}$, which corresponds to the strong-pump regime. The following important features are observed in this regime:

(a) The growth rate curves have sharp peaks at

$$
\Delta K^{\prime}=n \frac{2}{\pi K\left(\kappa_{M}\right)}, \text { where } n=1,2, \cdots,
$$

and the growth rate $\operatorname{Im}\left(\Delta \omega^{\prime}\right)$ is much smaller than $\hat{\omega}_{B}^{\prime}\left(\gamma_{M}^{\prime}\right)$.

(b) The normalized real frequency shift $\operatorname{Re}\left(\Delta \Omega^{\prime}\right)$ for the unstable sideband mode is nearly equal to $\Delta K^{\prime}$. Together with the sharp peaks at $\Delta K^{\prime}=n \frac{2}{\pi K\left(\kappa_{M}\right)}$, this implies that the instability has strong resonance features when the real frequency shift $\operatorname{Re}\left(\Delta \omega^{\prime}\right)$ is equal to harmonics of $\omega_{b}^{\prime}\left(\gamma_{M}^{\prime}\right)=\frac{2}{\pi K\left(\kappa_{M}\right)} \hat{\omega}_{B}^{\prime}\left(\gamma_{M}^{\prime}\right)$, which is the bounce frequency of electrons with energy $\gamma_{0}^{\prime}=\gamma_{M I}^{\prime}$. Therefore, in the strong-pump regime, it can be argued that the sideband instability is driven by the synchrotron oscillations of trapped electrons in the ponderomotive potential.

(c) For small values of $\kappa_{M}$, only the fundamental peak $(n=1)$ has a significant growth rate. As $\kappa_{M}$ is increased, the growth rate of the higher harmonics becomes comparable to the growth rate of the fundamental. However, the separation between adjacent peaks decreases and the maximum growth rate drops. When $\kappa_{M}$ is equal to unity, the distinct peaks coalesce into a single smooth curve, and the maximum growth rate decreases substantially relative to that obtained for deeply trapped electrons $\left(\kappa_{M} \simeq 0\right.$ and $\left.\gamma_{M}^{\prime} \simeq \hat{\gamma}_{-}^{\prime}\right)$

Shown in Figs.5 and 6 are the normalized growth rate $\operatorname{Im}\left(\Delta \Omega^{\prime}\right)=\operatorname{Im}\left(\Delta \omega^{\prime}\right) / \hat{\omega}_{B}^{\prime}\left(\gamma_{M}^{\prime}\right)$ and the corresponding real frequency shift $\operatorname{Re}\left(\Delta \Omega^{\prime}\right)=\operatorname{Re}\left(\Delta \omega^{\prime}\right) / \hat{\omega}_{B}^{\prime}\left(\gamma_{M}^{\prime}\right)$, respectively, plotted versus $\Delta K^{\prime}$ for the choice of pump strength $P=1.0$, which corresponds to 
intermediate pump strength. In this regime, the general features of the instability are:

(a) The growth rate curves have wider bandwidth than those in the strong-pump regime, and the maximum growth rate is comparable to $\dot{\omega}_{B}^{\prime}\left(\gamma_{M I}^{\prime}\right)$.

(b) The maximum growth rate does not occur exactly at harmonics of the bounce frequency $\omega_{b}^{\prime}\left(\gamma_{M I}^{\prime}\right)$. However, the sideband instability is still driven by the synchrotron oscillations of trapped electrons in the ponderomotive potential. Moreover, the growth of the sideband signal is so strong (with growth rate comparable to the real frequency shift) that the resonances broaden.

(c) The increase of $\kappa_{M}$ causes a decrease in the maximum growth rate and a corresponding merging of the peaks in the growth rate curves. This is similar to the behavior in the strong-pump regime.

Shown in Figs.7 and 8 are plots versus $\Delta K^{\prime}$ of the normalized growth rate $\operatorname{Im}\left(\Delta \Omega^{\prime}\right)=$ $\operatorname{Im}\left(\Delta \omega^{\prime}\right) / \hat{\omega}_{B}^{\prime}\left(\gamma_{M I}^{\prime}\right)$ and the corresponding real frequency shift $\operatorname{Re}\left(\Delta \Omega^{\prime}\right)=\operatorname{Re}\left(\Delta \omega^{\prime}\right) / \hat{\omega}_{B}^{\prime}\left(\gamma_{M}^{\prime}\right)$ respectively, for the choice of pump strength $P=10^{-3}$, which corresponds to the weak-pump regime. The general features of the stability behavior in this regime can be summarized as follows:

(a) No resonance peaks are observed. The normalized growth rate, real frequency shift and wavenumber at maximum growth agree with those predicted in Eqs.(146) and (147).

(b) Increasing the value $\kappa_{M}$ has little effect on the maximum growth rate, other than shifting the location on the $\Delta K^{\prime}$ axis where the maximum growth occurs.

Shown in Fig. 9 are the plots of the maximum growth rate versus the pump strength $P$, calculated from Eq.(141) for four different values of $\kappa_{M r}$. The same results are displayed in (a) a linear-scale plot, and (b) a log-scale plot. It is evident that the larger the pump strength $P$, the more the maximum growth rate is affected by the 
level of electron population $\left(\gamma_{M}^{\prime}\right.$ and $\left.\kappa_{M}\right)$ in the ponderomotive potential. 


\section{Conclusions}

In the present analysis, we have made use Vlasov-Maxwell equations to investigate detailed properties of the sideband instability for a helical wiggler free electron laser. The model describes the nonlinear evolution of a right-circularly polarized primary electromagnetic wave with frequency $\omega_{s}$, wavenumber $k_{s}$, and slowly varying amplitude $\hat{a}_{s}(z, t)$ and phase $\delta_{s}(z, t)$ (eikonal approximation). The theoretical model and the equilibrium analysis were described in Sec.II. It was found in quasi-steady state $\left(\partial / \partial t^{\prime}=0\right)$ that the phase of the primary electromagnetic wave has slow spatial variation [Eq.(28)]. In Sec.III, the formal kinetic dispersion relation [Eqs.(60) and (64)] was derived in terms of the dielectric functions $\alpha_{\nu}$ and $\chi_{\nu}^{ \pm}\left(\Delta \omega^{\prime}\right)$ [Eq.(56)].

In Sec.IV, we considered the case where the trapped electrons uniformly populate the ponderomotive potential up to the separatrix $\left(\gamma_{0}^{\prime}=\hat{\gamma}_{+}^{\prime}\right)$. Making use of the exact trajectory of an electron on the separatrix [Eq.(84)], the dispersion relation was obtained in closed form [Eq.(103)], and the maximum growth rate was determined analytically in the weak-pump limit $(P \ll 1)$. For general distribution function consisting of trapped electrons only, the dispersion relation was simplified to a tractable form [Eq.(130)] which involves integrals over only the variable $\gamma_{0}^{\prime}$ (Sec.V). In Sec.VI, a closed form for the dispersion relation was obtained for a step-function distribution [Eq.(132)], and in the weak-pump limit $(P \ll 1)$ the maximum growth rate was determined analytically. The closed dispersion relations (103) and (141) were solved numerically for different values of the dimensionless pump parameter $P$ and maximum energy $\gamma_{M}^{\prime}$, and detailed stability properties were examined. In was found in the strong-pump regime $(P \gg 1)$ and in the intermediate-pump regime $(P \simeq 1)$ that plots of the growth rate versus wavenumber exhibit a strong resonance structure, which indicates that the interaction between the sideband signal and the bounce motion of the trapped electrons is the driving mechanism for the instability. It was 
also observed in these regimes that an increase in $\gamma_{M}^{\prime}$ causes a decrease in the maximum growth rate and a decrease in separation between adjacent growth peaks, which coalesce in the limit $\gamma_{M I}^{\prime} \rightarrow \hat{\gamma}_{+}^{\prime}$. By contrast, in the weak-pump limit $(P \ll 1)$, no resonance structure was observed, and a change in $\gamma_{M}^{\prime}$ has little effect on the growth rate. The maximum growth rate in the weak-pump regime is determined analytically to be

$$
\operatorname{Im}\left(\Delta \omega^{\prime}\right)_{M}=\frac{\sqrt{3}}{2}\left(\Gamma_{0}^{\prime} c k_{p}^{\prime}\right)=\frac{3^{1 / 2}}{2}\left(\frac{\hat{\omega}_{p b}^{\prime 2} a_{w}^{2} c^{2} k_{p}^{\prime 2}}{4 \hat{\gamma}^{\prime 3} \omega_{s}^{\prime}}\right)^{1 / 3}
$$

which is independent of $\gamma_{M}^{\prime}$ and $\hat{a}_{s}^{0}$. It should be pointed out that if the smallsignal dispersion relation in the high-gain Compton regime [e.g., Eq.(26) in Ref.75 with $k_{e m} \simeq \omega / c$ and $F=1$ ] is transformed to the ponderomotive frame, and solved for complex frequency $\omega^{\prime}$ with real wavenumber $k^{\prime}$, the maximum growth rate is $\operatorname{Im}\left(\omega^{\prime}\right)_{M}=\frac{\sqrt{3}}{2}\left(\Gamma_{0}^{\prime} c k_{p}^{\prime}\right)$, the same as that obtained for the sideband instability in the weak-pump regime. This indicates that the mechanism responsible for the sideband instability in the weak-pump limit is similar to that of the usual free electron laser instability in the small-signal regime. The bounce motion of the trapped electrons in the ponderomotive frame plays a minor role in the weak-pump limit.

Although the dispersion relations (103) and (130) have been obtained in terms of "primed" (ponderomotive frame) variables $\Delta k^{\prime}$ and $\Delta \omega^{\prime}$ in the present analysis, these equations can be expressed in terms of laboratory frame variables $\Delta k$ and $\Delta \omega$ by means of the transformation

$$
\begin{aligned}
\Delta \omega^{\prime} & =\gamma_{p}\left(\Delta \omega-\nu_{p} \Delta k\right), \\
\Delta k^{\prime} & =\gamma_{p}\left(\Delta k-\frac{\nu_{p}}{c^{2}} \Delta \omega\right) .
\end{aligned}
$$

Here, for real $\Delta k^{\prime}$ and complex $\Delta \omega^{\prime}$, both $\Delta k$ and $\Delta \omega$ are complex. Also note that the characteristic time scale for the slow variation of system parameters is $1 / \hat{\omega}_{B}^{\prime}\left(\hat{\gamma}^{\prime}\right)$ [Eq.(78)], where $\hat{\omega}_{B}^{\prime}\left(\hat{\gamma}^{\prime}\right)$ is the bounce frequency of a deeply trapped electron with 
energy $\hat{\gamma}^{\prime}$. In the laboratory frame, the characteristic time scale is $1 / \Omega_{B}$, where

$$
\begin{aligned}
\Omega_{B}=\hat{\omega}_{B}^{\prime}\left(\hat{\gamma}^{\prime}\right) / \gamma_{p} & =\left(c^{2} k_{p}^{\prime 2} a_{w} \hat{a}_{s}^{0} / \hat{\gamma}^{\prime 2} \gamma_{p}^{2}\right)^{1 / 2} \\
& =\left(1+v_{p} / c\right)\left[a_{w} \hat{a}_{s}^{0} /\left(1+a_{w}^{2}\right)\right]^{1 / 2} c k_{0}
\end{aligned}
$$

is the characteristic bounce frequency in the laboratory frame. Here, $k_{p}^{\prime}$ has been approximated by $k_{p}^{\prime}=\left(k_{s}+k_{0}\right) / \gamma_{p}=\gamma_{p}\left(1+v_{p} / c\right) k_{0}$.

To summarize, a kinetic formalism which accommodates general electron distribution has been developed (Secs.II and III) for investigating detailed properties of the sideband instability over a wide range of system parameters. For a particular form of trapped-electron distribution function (i.e., a step-function distribution), the stability properties were analysed in detail. When the dimensionless pump strength $P$ is of order unity or larger, it was found that the maximum energy $\gamma_{M}^{\prime}$ of the trappedelectron population has a significant influence on stability properties. Moreover, the dispersion relation (130) can be used to study the sideband instability for general form of the trapped-electron distribution. A study of stability behavior for distribution functions $f^{0}\left(\gamma_{0}^{\prime}\right)$ that vary smoothly with $\gamma_{0}^{\prime}$ will be the subject of a future investigation, and it is expected that the form of the distribution function will change the resonance structure from that obtained in the present analysis, where $f^{0}\left(\gamma_{0}^{\prime}\right)$ is assumed to be a step function. Furthermore, while emphasis in the present analysis has been placed on the case where only trapped electrons are present (Secs.IV-VI), the general dispersion relation (60) can also be used to investigate detailed stability properties in circumstances where untrapped electrons play an important role in modifying detailed stability behavior. 


\section{Acknowledgments}

This research was supported by the National Science Foundation, and in part by the Department of Energy High Energy Physics Division and the Naval Research Laboratory Plasma Physics Division. The research by one of the authors (T.-Y. B. $\mathrm{Y}$.) was in partial fulfillment of the requirements for the $\mathrm{Ph} . \mathrm{D}$. degree. 


\section{References}

${ }^{1}$ C. A. Brau, IEEE J. Quantum Electron. QE-21, 824 (1985)

${ }^{2}$ T. J. Orzechowski, B. Anderson, W. M. Fawley, D. Prosnitz, E. T. Scharlemann, S. Yarema, D. B. Hopkins, A. C. Paul, A. M. Sessler and J. S. Wurtele, Phys. Rev. Lett. 54, 889 (1985).

${ }^{3}$ T. J. Orzechowski, E. T. Scharlemann, B. Anderson, V. K. Neil, W. M. Fawley, D. Prosnitz, S. M. Yarema, D. B. Hopkins, A. C. Paul, A. M. Sessler and J. S. Wurtele, IEEE J. Quantum Electron. QE-21, 831 (1985).

${ }^{4}$ M. Billardon, P. Elleaume, J. M. Ortega, C. Bazin, M. Bergher, M. Velghe, D. A. G.Deacon and Y. Petroff, IEEE J. Quantum Electron. QE-21, 805 (1985).

${ }^{5}$ R. W. Warren, B. E. Newnam and J. C. Goldstein, IEEE J. Quantum Electron. QE-21, 882 (1985).

${ }^{6}$ J. Masud, T. C. Marshall, S. P. Schlesinger and F. G. Yee, Phys. Rev. Lett. 56, 1567 (1986).

${ }^{7} J$. Masud, T. C. Marshall, S. P. Schlesinger, F. G. Yee, W. M. Fawley E. T. Scharlemann, S. S. Yu, A. M. Sessler and E. J. Sternbach, Phys. Rev. Lett. 58, 763 (1987).

${ }^{8}$ F. G. Yee, T. C. Marshall and S. P. Schlesinger, IEEE Trans. Plasma Sci. PS-16, $162(1988)$.

${ }^{9}$ J. Fajans, G. Bekef, Y. Z. Yin and B. Lax, Phys. Rev. Lett. 53, 246 (1984).

${ }^{10}$ R. W. Warren, B. E. Newnam, J. G. Winston, W. E. Stein, L. M. Young and C. A. Brau, IEEE J. Quantum Electron. QE-19, 391 (1983).

${ }^{11}$ G. Bekefi, R. E. Shefer and W. W. Destler, Appl. Phys. Lett. 44, 280 (1983).

${ }^{12}$ C. W. Roberson, J. A. Pasour, F. Mako, R. F. Lucey,Jr. and P. Sprangle, Infrared Millimeter Waves 10, 361 (1983), and references therein.

${ }^{13}$ A. Grossman, T. C. Marshall and S. P. Schlesinger, Phys. Fluids 26, 337 (1983).

${ }^{14}$ D. Prosnitz and A. M. Sessler, In Physics of Quantum Electronics (Addison-Wesley, Reading, Massachusetts, 1982), Vol.9, p.651. 
${ }^{15}$ R. K. Parker, R. H. Jackson, S. H. Gold, H. P. Freund, V. L. Granatstein, P. C. Efthimion, M. Herndon and A. K. Kinkead, Phys. Rev. Lett. 48, 238 (1982).

${ }^{16}$ S. Benson, D. A. G. Deacon, J. N. Eckstein, J. M. J. Madey, K. Robinson, T. I. Smith and R. Taber, Phys. Rev. Lett, 48A, 235 (1982)

${ }^{17}$ A. N. Didenko, A. R. Borisov, G. R. Fomenko, A. V. Kosevnikov, G. V. Melnikov, I'u G. Stein and A. G. Zerlitsin, IEEE Trans. Nucl. Sci. NS-28, 3169 (1981).

${ }^{18}$ D. B. McDermott, T. C. Marshall, S. P. Schlesinger, R. K. Parker and V. L. Granatstein, Phys. Rev. Lett. 41, 1368 (1978).

${ }^{19}$ D. A. G. Deacon, L. R. Elias, J. M. J. Madey, G. J. Ramian, H. A. Schwettman and T. I. Smith, Phys. Rev. Lett. 38, 892 (1977).

${ }^{20}$ L. R. Elias, W. M. Fairbank, J. M. J. Madey, H. A. Schwettman and T. I. Smith, Phys. Rev. Lett. 36, 717 (1976).

${ }^{21}$ R. C. Davidson, Phys. Fluids 29, 2689 (1986).

${ }^{22}$ R. C. Davidson, J. S. Wurtele, and R. E. Aamodt, Phys. Rev. A 34,3063 (1986).

${ }^{23}$ B. Lane and R. C. Davidson, Phys. Rev. A 27, 2008 (1983).

${ }^{24}$ R. C. Davidson and J. S. Wurtele, Phys. Fluids 30, 577 (1987).

${ }^{25}$ R. C. Davidson and J. S. Wurtele, Phys. Fluids 30, 2825 (1987).

${ }^{26}$ S. Riyopoulos and C. M. Tang, Phys. Fluids 31, 1708 (1988).

${ }^{27}$ S. Riyopoulos and C. M. Tang, Phys. Fluids 31, 3387 (1988).

${ }^{28}$ W. M. Sharp and S. S. Yu, Phys. Fluids B2, to be published (1990).

${ }^{29}$ A. M. Dimos and R. C. Davidson, Phys. Fluids 28, 667 (1985).

${ }^{30}$ R. C. Davidson and Y. Z. Yin, Phys. Fluids 28, 2524 (1985).

${ }^{31}$ T. Taguchi, K. Mima and T. Mochizuki, Phys. Rev. Lett. 46, 824 (1981).

${ }^{32}$ F. A. Hopf, P. Meystre, M. O. Scully and W. II. Louisell, Phys. Rev. Lett. 37, 1342 (1976).

${ }^{33}$ R. C. Davidson and W. A. McMullin, Phys. Rev. A 26, 410 (1982). 
${ }^{34}$ N. S. Ginzburg and M. A. Shapiro, Opt. Commun. 40, 215 (1982).

${ }^{35} \mathrm{~J}$. C. Goldstein and W. B. Colson, in Proceedings of the International Conference on Lasers (STS, McLean, VA, 1982), p.218.

${ }^{36}$ W. B. Colson, IEEE J. Quantum Electron. QE-17, 1417 (1981).

${ }^{37}$ P. Sprangle, C. M. Tang and W. M. Manheimer, Phys. Rev. A 21, 302 (1980).

${ }^{38}$ W. H. Louisell, J. F. Lam, D. A. Copeland and W. B. Colson, Phys. Rev. A 19 , 288 (1979).

${ }^{39}$ N. M. Kroll, P. L. Morton and M. N. Rosenbluth, IEEE J. Quantum Electron. QE-17, 1436 (1981).

${ }^{40}$ D. C. Quimby, J. M. Slater and J. P. Wilcoxon, IEEE J. Quantum Electron. QE-21, 979 (1985).

${ }^{41}$ N. S. Ginzburg and M. I. Petelin, Int. J. Electron. 59, 291 (1985).

${ }^{42}$ C. M. Tang and P. Sprangle, in Free Electron Generators of Coherent Radiation, Edited by C. A. Brau, S. F. Jacobs and M. O. Scully (Society of Optical Engineering, Bellingham, WA, 1983), Vol.453, P.11.

${ }^{43}$ G. Dattoli, A. Marino, A. Renieri and F. Romanelli, IEEE J. Quantum Electron. QE-17, 1371 (1981).

${ }^{44}$ W. B. Colson, in Proceedings of the Seventh International Conference on Free Electron Lasers, edited by E. T. Scharlemann and D. Prosnitz (North-Holland, Amsterdam, 1986), p.168.

${ }^{45}$ M. N. Rosenbluth, H. V. Wong and B. N. Moore, in Ref.42, p. 25.

${ }^{46}$ A. T. Lin, Phys. Quantum Electron. 9, 867 (1982).

${ }^{47}$ H. Al-Abawi, J. K. McIver, G. T. Moore and M. O. Scully, Phys. Quantum Electron. 8, 415 (1982).

${ }^{48}$ W. B. Colson, Phys. Quantum Electron, 8, 457 (1982).

${ }^{49} \mathrm{~J}$. Goldstein, in Ref.42, p.2.

${ }^{50}$ R. C. Davidson and Y. Z. Yin, Phys. Rev. A 30, 3078 (1984). 
${ }^{51}$ G. L. Johnston and R. C. Davidson, J. Appl. Phys. 55, 1285 (1984).

${ }^{52}$ H. P. Freund and A. K. Ganguly, Phys. Rev. A 28, 3438 (1983).

${ }^{53}$ H. S. Uhm and R. C. Davidson, Phys. Fluids 26, 288 (1983).

${ }^{54}$ R. C. Davidson and H. S. Uhm, J. Appl. Phys. 53, 2910 (1982).

${ }^{55}$ H. S. Uhm and R. C. Davidson, Phys. Fluids 24, 2348 (1981).

${ }^{56}$ R. C. Davidson, W. A. McMullin and K.Tsang, Phys. Fluids 27, 233 (1983).

${ }^{57}$ R. C. Davidson and W. A. McMullin, Phys. Fluids 26, 840 (1983).

${ }^{58}$ W. A. McMullin and G. Bekefi, Phys. Rev. A 25, 1826 (1982).

${ }^{59}$ R. C. Davidson and W. A. McMullin, Phys. Rev. A 26, 1997 (1982).

${ }^{60}$ W. A. McMullin and G. Bekefi, Appl. Phys. Lett. 39, 845 (1981).

${ }^{61}$ R. C. Davidson, Phys. Fluids 29, 267 (1986).

${ }^{62}$ R. C. Davidson and J. S. Wurtele, IEEE Trans. Plasma Sci. PS-13, 464 (1985).

${ }^{63}$ H. P. Freund, R. A. Kehs and V. L. Granatstein, IEEE J. Quantum Electron. QE-21, 1080 (1985).

${ }^{64}$ H. P. Freund and A. K. Ganguly, IEEE J. Quantum Electron. QE-21, 1073 (1985).

${ }^{65}$ B. Hafizi and R. E. Aamodt, Phys. Rev. A 29, 2656 (1984).

${ }^{66}$ P. Sprangle, C. M. Tang and I. Bernstein, Phys. Rev. A 28, 2300 (1983).

${ }^{67}$ H. P. Freund and P. Sprangle, Phys. Rev. A 28, 1835 (1983).

${ }^{68}$ R. C. Davidson and H. S. Uhm, Phys. Fluids 23, 2076 (1980).

${ }^{69}$ P. Sprangle and R. A. Smith, Phys. Rev. A 21, 293 (1980).

${ }^{70}$ I. B. Bernstein and J. L. Hirshfield, Physica (Utrecht) A 20, 1661 (1979).

${ }^{71}$ T. Kwan and J. M. Dawson, Phys. Fluids 22, 1089 (1979).

${ }^{72}$ T. Kwan, J. M. Dawson and A. T. Lin, Phys. Fluids 20, 581 (1977). 
${ }^{73}$ A. Bambini and A. Renieri, Nuovo Cimeto Lett. 21, 399(1978).

${ }^{74}$ S. T. Stenholm and A. Bambini, IEEE J. Quantum Electron. QE-17, 1363(1981).

${ }^{75}$ C. W. Roberson and P. Sprangle, Phys. Fluids B1, 3 (1989).

${ }^{76}$ R. C. Davidson, Physics of Nonneutral Plasmas (Addison-Wesley, Reading, Massachusetts, 1990), Chapter 7.

${ }^{77}$ N. M. Kroll and W. A. McMullin, Phys. Rev. A 17, 300 (1978)

${ }^{78}$ A. Hasegawa, Bell Syst. Tech. J. 57, 3069 (1978).

${ }^{79}$ W. B. Colson, Phys. Lett. A 59, 187 (1976).

${ }^{80}$ V. P. Sukhatme and P. A. Wolff, J. Appl. Phys. 44, 2331 (1973).

${ }^{81}$ I. S. Gradshteyn and I. M. Ryzhik, Table of Integrals, Series and Products (Academic, New York, 1980).

${ }^{82} \mathrm{E}$. T. Whittaker and G. N. Watson, A Course of Modern Analysis, 4th ed., (Cambridge University Press, 1927, reprinted 1973).

${ }^{83}$ R. V. Churchill, J. W. Brown and R. F. Verhey, Complex Variables and Applications (McGraw-Hill, New York, 1974). 


\section{Appendix A. Derivation of Equations (96) and (99)}

Making the change of variable $u=\tan \left[\left(k_{p}^{\prime} z^{\prime}+\pi\right) / 4\right]$ gives

$$
\begin{aligned}
& \cos \left(k_{p}^{\prime} z^{\prime} / 2\right)=\sin \left[\left(k_{p}^{\prime} z^{\prime}+\pi\right) / 2\right]=\frac{2 u}{1+u^{2}} \\
& \exp \left( \pm i k_{p}^{\prime} z^{\prime}\right)=-\left(\frac{1-u^{2}}{1+u^{2}} \pm i \frac{2 u}{1+u^{2}}\right)^{2}=-\left(\frac{2 i}{u \pm i} \mp 1\right)^{2} .
\end{aligned}
$$

Making use of Eq.(84), it can also be shown that

$$
\exp \left[i k_{p}^{\prime} z_{ \pm}^{\prime \prime}\left(\tau^{\prime}\right)\right]=-\left(\frac{2 i \Gamma_{ \pm}}{u+i \Gamma_{ \pm}}-1\right)^{2}
$$

where

$$
\Gamma_{ \pm}=\exp \left(\mp \frac{\tau^{\prime}}{\tau_{B}}\right)
$$

After some straightforward algebra, it follows from Eqs.(A1) and (A2) that

$$
\begin{aligned}
& \int_{-\pi}^{\pi} d\left(k_{p}^{\prime} z^{\prime}\right) \frac{\exp \left(-i k_{p}^{\prime} z^{\prime}\right)}{\cos \left(k_{p}^{\prime} z^{\prime} / 2\right)}\left\{\exp \left(i k_{p}^{\prime} z^{\prime}\right)-\exp \left[i k_{p}^{\prime} z_{ \pm}^{\prime \prime}\left(\tau^{\prime}\right)\right]\right\} \\
& \quad=8\left(\Gamma_{ \pm}-1\right) i \int_{0}^{\infty} d u\left[1+\frac{4 i}{u-i}-\frac{4}{(u-i)^{2}}\right] \frac{u^{2}+\Gamma_{ \pm}}{(u+i)^{2}\left(u+i \Gamma_{ \pm}\right)^{2}}
\end{aligned}
$$

Carrying out the integral over $u$, Eq.(A4) reduces to

$$
\begin{aligned}
\int_{-\pi}^{\pi} d\left(k_{p}^{\prime} z^{\prime}\right) \frac{\exp \left(-i k_{p}^{\prime} z^{\prime}\right)}{\cos \left(k_{p}^{\prime} z^{\prime} / 2\right)}\left\{\exp \left(i k_{p}^{\prime} z^{\prime}\right)-\exp \left[i k_{p}^{\prime} z_{ \pm}^{\prime \prime}\left(\tau^{\prime}\right)\right]\right\} \\
=\frac{32\left(\Gamma_{ \pm}-1\right) \Gamma_{ \pm}}{\left(\Gamma_{ \pm}+1\right)^{3}} \ln \Gamma_{ \pm}+\frac{16\left(\Gamma_{ \pm}-1\right)^{2}}{\left(\Gamma_{ \pm}+1\right)^{2}}+i \frac{32\left(\Gamma_{ \pm}-1\right) \Gamma_{ \pm} \pi}{\left(\Gamma_{ \pm}+1\right)^{3}} \\
=\frac{32(\Gamma-1) \Gamma}{(\Gamma+1)^{3}} \ln \Gamma+\frac{16(\Gamma-1)^{2}}{(\Gamma+1)^{2}} \pm i \frac{32(\Gamma-1) \Gamma \pi}{(\Gamma+1)^{3}}
\end{aligned}
$$

where $\Gamma \equiv \Gamma_{+}$. Similarly it can be shown that

$$
\begin{aligned}
& \int_{-\pi}^{\pi} d\left(k_{p}^{\prime} z^{\prime}\right) \frac{\exp \left(i k_{p}^{\prime} z^{\prime}\right)}{\cos \left(k_{p}^{\prime} z^{\prime} / 2\right)}\left\{\exp \left(i k_{p}^{\prime} z^{\prime}\right)-\exp \left[i k_{p}^{\prime} z_{ \pm}^{\prime \prime}\left(\tau^{\prime}\right)\right]\right\} \\
& \quad=8\left(\Gamma_{ \pm}-1\right) i \int_{0}^{\infty} d u\left[1-\frac{4 i}{u+i}-\frac{4}{(u+i)^{2}}\right] \frac{u^{2}+\Gamma_{ \pm}}{(u+i)^{2}\left(u+i \Gamma_{ \pm}\right)^{2}} \\
& \quad=16\left[\frac{\Gamma_{ \pm}^{2}+10 \Gamma_{ \pm}+1}{3\left(\Gamma_{ \pm}-1\right)^{2}}-\frac{2 \Gamma_{ \pm}\left(\Gamma_{ \pm}+1\right)}{\left(\Gamma_{ \pm}-1\right)^{3}} \ln \Gamma_{ \pm}\right] \\
& \quad=16\left[\frac{\Gamma^{2}+10 \Gamma+1}{3(\Gamma-1)^{2}}-\frac{2 \Gamma(\Gamma+1)}{(\Gamma-1)^{3}} \ln \Gamma\right]
\end{aligned}
$$




\section{Appendix B. Dielectric Responses for Uniformly-Populated Trapped-Electron Distribution}

Making the change of variable $x=-\tau^{\prime} / \tau_{B}$ gives

$$
\begin{gathered}
\int_{-\infty}^{0} d \tau^{\prime} \exp \left(-i \Delta \omega^{\prime} \tau^{\prime}\right)\left[\frac{2 \Gamma(\Gamma-1)}{(\Gamma+1)^{3}} \ln \Gamma+\frac{(\Gamma-1)^{2}}{(\Gamma+1)^{2}}\right] \\
=\frac{i}{\Delta \omega^{\prime}}-4 \tau_{B} \int_{0}^{\infty} d x \frac{\exp [(i \alpha+1) x]}{[\exp (x)+1]^{2}} \\
+2 \tau_{B} \int_{0}^{\infty} d x \frac{x \exp [(i \alpha+1) x][\exp (x)-1]}{[\exp (x)+1]^{3}}
\end{gathered}
$$

where $\alpha=\Delta \omega^{\prime} \tau_{B}$. To express Eq.(B1) in a more compact form, we define

$$
Y(\alpha) \equiv \int_{0}^{\infty} d x \frac{\exp (i \alpha x)}{\exp (x)+1}, \quad \operatorname{Im} \alpha>-1
$$

Integrating by parts, it can be shown that

$$
\begin{aligned}
\int_{0}^{\infty} d x \frac{\exp [(i \alpha+1) x]}{[\exp (x)+1]^{2}} & =\frac{1}{2}+i \alpha Y(\alpha) \\
\int_{0}^{\infty} d x \frac{x \exp [(i \alpha+1) x][\exp (x)-1]}{[\exp (x)+1]^{3}} & =\frac{1}{2}+2 i \alpha Y(\alpha)+i \alpha^{2} \frac{d}{d \alpha} Y(\alpha) .
\end{aligned}
$$

Substituting Eq.(B3) into Eq.(B1), it follows from Eq.(98) that

$$
\hat{\omega}_{p b}^{\prime 2} \alpha_{0}-\chi_{0}^{+}\left(\Delta \omega^{\prime}\right)=-\frac{a_{w} \hat{\omega}_{p b}^{\prime 2}}{2 \hat{\gamma}_{+}^{\prime} \hat{a}_{s}^{0}}\left\{1+i \alpha\left[1-2 i \alpha^{2} \frac{d}{d \alpha} Y(\alpha)\right]\right\},
$$

where $\alpha=\Delta \omega^{\prime} \tau_{B}$. Equation (B4) is valid for $\operatorname{Im} \alpha>-1$. Moreover, the analytic continuation of $Y(\alpha)$ into the region of the complex $\alpha$-plane where $\operatorname{Im} \alpha<-1$ can be obtained by deformation of the integration contour in Eq.(B2). Without presenting the detailed derivation, the result is

$$
Y(\alpha)= \begin{cases}\int_{0}^{\infty} d x \frac{\exp (i \alpha x)}{\exp (x)+1}, & \operatorname{Im} \alpha>-1 \\ \int_{0}^{\infty} d x \frac{\exp (-i \alpha x)}{\exp (x)+1}+\frac{i}{\alpha}-\frac{\pi i}{\sinh (\pi \alpha)}, & \operatorname{Im} \alpha \leq-1\end{cases}
$$

The function $Y(\alpha)$ defined in Eq.(B5) is analytic over the entire complex $\alpha$-plane except for the discrete poles located at $\alpha=-i n$, where $n=1,2,3 \cdots$. It can also be 
shown that $Y(\alpha)$ has the recursive property

$$
Y(\alpha-i)=\frac{i}{\alpha}-Y(\alpha)
$$

over the entire complex $\alpha$-plane.

Similarly, it can be shown that

$$
\begin{gathered}
\int_{-\infty}^{0} d \tau^{\prime} \exp \left(-i \Delta \omega^{\prime} \tau^{\prime}\right)\left[\frac{\Gamma^{2}+10 \Gamma+1}{3(\Gamma-1)^{2}}-\frac{2 \Gamma(\Gamma+1)}{(\Gamma-1)^{3}} \ln \Gamma\right] \\
=\frac{i}{3 \Delta \omega^{\prime}}+2 \tau_{B} \int_{0}^{\infty} d x \exp (i \alpha x) \frac{\exp (x)}{[\exp (x)-1]^{3}} \\
\times\{2[\exp (x)-1]-x[\exp (x)+1]\}
\end{gathered}
$$

Integrating by parts, it follows from Eqs.(100) and (B7) that

$$
\hat{\omega}_{p b}^{\prime 2} \alpha_{2}-\chi_{2}^{+}\left(\Delta \omega^{\prime}\right)=-\frac{a_{w} \hat{\omega}_{p b}^{\prime 2}}{2 \hat{\gamma}_{+}^{\prime} \hat{a}_{s}^{0}}\left\{\frac{1}{3}-i \alpha\left[2 \alpha^{2} Z(\alpha)-(2 i \alpha+1)\right]\right\},
$$

where $Z(\alpha)$ is defined by

$$
Z(\alpha) \equiv \int_{0}^{\infty} d x \frac{x \exp (i \alpha x)}{\exp (x)-1}, \quad \operatorname{Im} \alpha>-1
$$

Extending the definition of $Z(\alpha)$ to the entire complex $\alpha$-plane by analytic continuation gives

$$
Z(\alpha)= \begin{cases}\int_{0}^{\infty} d x \frac{x \exp (i \alpha x)}{\exp (x)-1}, & \operatorname{Im} \alpha>-1 \\ -\int_{0}^{\infty} d x \frac{x \exp (-i \alpha x)}{\exp (x)-1}+\frac{1}{\alpha^{2}}-\frac{\pi^{2}}{\sinh ^{2}(\pi \alpha)}, & \operatorname{Im} \alpha \leq-1\end{cases}
$$

The function $Z(\alpha)$ has the recursive property

$$
Z(\alpha-i)=-\frac{1}{\alpha^{2}}+Z(\alpha)
$$

over the entire complex $\alpha$-plane. 


\section{Appendix C. Maximum Growth Rate for a Uniformly- Populated Trapped-Electron Distribution in the Weak-Pump Limit}

To determine the maximum growth rate in the weak-pump limit $(P \ll 1)$ from Eq.(107), we investigate two limiting cases.

Case I: $\left|\Delta K^{\prime}\right| \gg\left|\Delta \Omega^{\prime}\right| \gg 1$

In this limit, the leading order solution to Eq.(107) is

$$
\Delta K^{\prime}=\frac{1}{3 P^{1 / 2}}
$$

Correct to the next order, Eq.(107) can be approximated by

$$
\frac{1}{9 P}\left(1-6 \Delta \Omega^{\prime} P^{1 / 2}\right)=\frac{1}{9 P}\left[1+\frac{3}{\left(\Delta \Omega^{\prime}\right)^{2}}\right] .
$$

Solving Eq.(C2) for $\Delta \Omega^{\prime}$ gives

$$
\left(\Delta \Omega^{\prime}\right)_{M}=\frac{1}{2^{1 / 3} P^{1 / 6}}\left(\frac{1}{2}+i \frac{\sqrt{3}}{2}\right)
$$

Case II: $\left|\Delta \Omega^{\prime}\right| \gg\left|\Delta K^{\prime}\right|,\left|\Delta \Omega^{\prime}\right| \gg 1$

In this limit, the leading order solution to Eq.(107) is

$$
\Delta \Omega^{\prime}=\frac{1}{3 P^{1 / 2}}
$$

We express $\Delta \Omega^{\prime}$ as

$$
\Delta \Omega^{\prime}=\frac{1}{3 P^{1 / 2}}+\delta \Omega^{\prime}
$$

Correct to the next order, Eq.(107) can be approximated by

$$
\frac{1}{9 P}\left[1+6 P^{1 / 2}\left(\delta \Omega^{\prime}-\Delta K^{\prime}\right)\right]=\frac{1}{9 P}(1+27 P) .
$$

Solving for $\Delta \Omega^{\prime}$ gives

$$
\delta \Omega=\Delta K^{\prime}+\frac{9}{2} P^{1 / 2}
$$


Since $\Delta \Omega^{\prime}$ is real-valued, correct to order $P^{1 / 2}$, the maximum growth rate must be smaller than $P^{1 / 2}$ in the weak-pump limit $(P \ll 1)$. This implies that the growth rate for Case I is larger than that for Case II.

After examining other possible cases, we conclude that the maximum growth rate obtained from Eq.(107) occurs in the limit described in Case I, and the corresponding growth rate and real frequency shift are given in Eq.(C3).

\section{Appendix D. Maximum Growth Rate for a Step-Function Trapped-Electron Distribution in the Weak- Pump Limit}

The condition for Eq.(145) to be a good approximation to Eq.(141) is

$$
Q_{l}^{ \pm}\left(\kappa_{M}\right) \ll G_{M}\left(\gamma_{M}^{\prime}\right), \quad \text { for } \quad|l| \gtrsim\left|\frac{2 K\left(\kappa_{M}\right)}{\pi} \Delta \Omega^{\prime}\right|
$$

which will be shown to be satisfied at maximum growth in the weak-pump limit.

Similar to appendix C, we investigate Eq.(145) in two limiting cases.

Case I: $\left|\Delta K^{\prime}\right| \gg\left|\Delta \Omega^{\prime}\right| \gg 1$

In this limit, the leading order solution to Eq.(145) is

$$
\Delta K^{\prime}=\frac{G_{M}\left(\gamma_{M I}^{\prime}\right)}{P^{1 / 2}}
$$

Correct to the next order, Eq.(145) can be approximated by

$$
\frac{G_{M I}^{2}\left(\gamma_{M}^{\prime}\right)}{P}\left[1-\frac{2 P^{1 / 2}}{G_{M}\left(\gamma_{M}^{\prime}\right)} \Delta \Omega^{\prime}\right]=\frac{G_{M I}^{2}\left(\gamma_{M I}^{\prime}\right)}{P}\left[1+\frac{1}{G_{M}\left(\gamma_{M}^{\prime}\right)} \frac{1}{\left(\Delta \Omega^{\prime}\right)^{2}}\right]
$$

In obtaining Eq.(D3) use has been made of Eq.(144). Solving for $\Delta \Omega^{\prime}$ from Eq.(D3) gives

$$
\left(\Delta \Omega^{\prime}\right)_{M}=\frac{1}{2^{1 / 3} P^{1 / 6}}\left(\frac{1}{2}+i \frac{\sqrt{3}}{2}\right)
$$


Case II: $\left|\Delta \Omega^{\prime}\right| \gg\left|\Delta K^{\prime}\right|,\left|\Delta \Omega^{\prime}\right| \gg 1$

In this limit, the leading order solution to Eq.(145) is

$$
\Delta \Omega^{\prime}=\frac{G_{M}\left(\gamma_{M I}^{\prime}\right)}{P^{1 / 2}}
$$

We express $\Delta \Omega^{\prime}$ as

$$
\Delta \Omega^{\prime}=\frac{G_{M I}\left(\gamma_{M}^{\prime}\right)}{P^{1 / 2}}+\delta \Omega^{\prime}
$$

Correct to the next order, Eq.(145) can be approximated by

$$
\frac{G_{M I}^{2}\left(\gamma_{M}^{\prime}\right)}{P}\left[1+\frac{2 P^{1 / 2}}{G_{M}\left(\gamma^{\prime}\right)}\left(\delta \Omega^{\prime}-\Delta K^{\prime}\right)\right]=\frac{G_{M}^{2}\left(\gamma_{M}^{\prime}\right)}{P}\left[1+\frac{P}{G_{M}^{3}\left(\gamma_{M}^{\prime}\right)}\right]
$$

Solving for $\Delta \Omega^{\prime}$ gives

$$
\delta \Omega^{\prime}=\Delta K^{\prime}+\frac{P^{1 / 2}}{2 G_{M}^{2}\left(\gamma_{M}^{\prime}\right)} .
$$

Since $\Delta \Omega^{\prime}$ is real-valued, correct to order $P^{1 / 2}$, the maximum growth rate must be smaller than $P^{1 / 2}$ in the weak-pump limit $(P \ll 1)$. This implies that the growth rate for Case I is larger than that for Case II.

After investigating other possible cases, we conclude that the maximum growth rate obtained from Eq.(145) occurs in the limit described in Case I, and the corresponding growth rate and real frequency shift are given in Eq.(D4).

Making use of Eq.(D.4), the condition in Eq.(D1) can be expressed as

$$
Q_{l}^{ \pm}\left(\kappa_{M}\right) \ll G_{M}\left(\gamma_{M}^{\prime}\right), \quad \text { for } \quad|l| \gtrsim \frac{2 K\left(\kappa_{M}\right)}{\pi} \frac{1}{2^{1 / 3} P^{1 / 6}} .
$$

For specified value of $\gamma_{M}^{\prime}$, it is evident that the condition in Eq.(D9) can be satisfied for sufficiently small values of the dimensionless pump strength $P$. 


\section{FIGURE CAPTIONS}

Fig.1 In the equilibrium state, the electron motion in the phase space $\left(z^{\prime}, p_{z}^{\prime}\right)$ occurs on surfaces with $\gamma_{0}^{\prime}=$ const, where $\gamma_{0}^{\prime}$ is defined in Eq.(29).

Fig.2 Schematic of trapped-electron distribution function $f^{0}\left(\gamma_{0}^{\prime}\right)$ versus $\gamma_{0}^{\prime}$, for the case in which electrons uniformly populate the ponderomotive potential up to an energy $\gamma_{M}^{\prime} \leq \hat{\gamma}_{+}^{\prime}$.

Fig.3 Plots of the normalized growth rate $\operatorname{Im}\left(\Delta \Omega^{\prime}\right)=\operatorname{Im}\left(\Delta \omega^{\prime}\right) / \hat{\omega}_{B}^{\prime}\left(\gamma_{M}^{\prime}\right)$ versus normalized wavenumber $\Delta K^{\prime}=v_{g}^{\prime} \Delta k^{\prime} / \hat{\omega}_{B}^{\prime}\left(\gamma_{M}^{\prime}\right)$ obtained from Eq.(141) for the choice of pump parameter $P=10^{3}$, and several values of $\kappa_{M}$ corresponding to $(a) \kappa_{M}=0.1,(b) \kappa_{M}=0.7,(c) \kappa_{M}=0.9,(d) \kappa_{M}=0.99$, and $(e) \kappa_{M}=0.999$. Shown in (f) is the result corresponding to $\kappa_{M}=1.0$, which is obtained from Eq.(103) for $P=10^{3}$.

Fig.4 Plots of the normalized real frequency shift $\operatorname{Re}\left(\Delta \Omega^{\prime}\right)=\operatorname{Re}\left(\Delta \omega^{\prime}\right) / \hat{\omega}_{B}^{\prime}\left(\gamma_{M}^{\prime}\right)$ versus normalized wavenumber $\Delta K^{\prime}=v_{g}^{\prime} \Delta k^{\prime} / \hat{\omega}_{B}^{\prime}\left(\gamma_{M}^{\prime}\right)$ obtained from Eq.(141) for the choice of pump parameter $P=10^{3}$, and several values of $\kappa_{M}$ corresponding to $(a) \kappa_{M}=0.1,(b) \kappa_{M}=0.7,(c) \kappa_{M}=0.9,(d) \kappa_{M}=0.99$, and $(\dot{e}) \kappa_{M}=0.999$. Shown in (f) is the result corresponding to $\kappa_{M}=1.0$, which is obtained from Eq.(103) for $P=10^{3}$.

Fig.5 Plots of the normalized growth rate $\operatorname{Im}\left(\Delta \Omega^{\prime}\right)=\operatorname{Im}\left(\Delta \omega^{\prime}\right) / \hat{\omega}_{B}^{\prime}\left(\gamma_{M}^{\prime}\right)$ versus normalized wavenumber $\Delta K^{\prime}=v_{g}^{\prime} \Delta k^{\prime} / \dot{\omega}_{B}^{\prime}\left(\gamma_{M}^{\prime}\right)$ obtained from Eq.(141) for the choice of pump parameter $P=1.0$, and several values of $\kappa_{M}$ corresponding to $(a) \kappa_{M}=0.1,(b) \kappa_{M}=0.7,(c) \kappa_{M}=0.9,(d) \kappa_{M}=0.99$, and $(e) \kappa_{M}=0.999$. Shown in (f) is the result corresponding to $\kappa_{M}=1.0$, which is obtained from Eq.(103) for $P=1.0$. 
Fig.6 Plots of the normalized real frequency shift $\operatorname{Re}\left(\Delta \Omega^{\prime}\right)=\operatorname{Re}\left(\Delta \omega^{\prime}\right) / \hat{\omega}_{B}^{\prime}\left(\gamma_{M}^{\prime}\right)$ versus normalized wavenumber $\Delta K^{\prime}=v_{g}^{\prime} \Delta k^{\prime} / \dot{\omega}_{B}^{\prime}\left(\gamma_{M}^{\prime}\right)$ obtained from Eq.(141) for the choice of pump parameter $P=1.0$, and several values of $\kappa_{M}$ corresponding to $(a) \kappa_{M}=0.1,(b) \kappa_{M}=0.7,(c) \kappa_{M}=0.9,(d) \kappa_{M}=0.99$, and $(e) \kappa_{M}=0.999$. Shown in $(\mathfrak{f})$ is the result corresponding to $\kappa_{M}=1.0$, which is obtained from Eq.(103) for $P=1.0$.

Fig.7 Plots of the normalized growth rate $\operatorname{Im}\left(\Delta \Omega^{\prime}\right)=\operatorname{Im}\left(\Delta \omega^{\prime}\right) / \hat{\omega}_{B}^{\prime}\left(\gamma_{M}^{\prime}\right)$ versus normalized wavenumber $\Delta K^{\prime}=v_{g}^{\prime} \Delta k^{\prime} / \hat{\omega}_{B}^{\prime}\left(\gamma_{M}^{\prime}\right)$ obtained from Eq.(141) for the choice of pump parameter $P=10^{-3}$, and several values of $\kappa_{M}$ corresponding to $(a) \kappa_{M}=0.1,(b) \kappa_{M}=0.7,(c) \kappa_{M}=0.9,(d) \kappa_{M}=0.99$, and $(e) \kappa_{M}=0.999$. Shown in (f) is the result corresponding to $\kappa_{M}=1.0$, which is obtained from Eq.(103) for $P=10^{-3}$.

Fig.8 Plots of the normalized real frequency shift $\operatorname{Re}\left(\Delta \Omega^{\prime}\right)=\operatorname{Re}\left(\Delta \omega^{\prime}\right) / \hat{\omega}_{B}^{\prime}\left(\gamma_{M}^{\prime}\right)$ versus normalized wavenumber $\Delta K^{\prime}=v_{g}^{\prime} \Delta k^{\prime} / \hat{\omega}_{B}^{\prime}\left(\gamma_{M}^{\prime}\right)$ obtained from Eq.(141) for the choice of pump parameter $P=10^{-3}$, and several values of $\kappa_{M},(a) \kappa_{M}=$ $0.1,(b) \kappa_{M I}-0.7,(c) \kappa_{M I}-0.9 .(d) \kappa_{M} \ldots 0.99$, and $(c) \kappa_{M}=0.999$. Shown in (f) is the result corresponding to $\kappa_{M}-1.0$, which is obtained from Eq.(103) for $P=10^{-3}$.

Fig.9 Plots of the normalized maximum growth rate $\frac{2^{4 / 3}}{3^{1 / 2}} P^{1 / 6} \operatorname{Im}\left(\Delta \omega^{\prime}\right)_{M} / \hat{\omega}_{B}^{\prime}\left(\gamma_{M I}^{\prime}\right)=$ $\operatorname{Im}\left(\Delta \omega^{\prime}\right)_{M} /\left(\frac{\sqrt{ } 3}{2} \Gamma_{0}^{\prime} c k_{p}^{\prime}\right)$ versus pump strength $P^{1 / 6}=\dot{\omega}_{B}^{\prime}\left(\hat{\gamma}^{\prime}\right) /\left(2^{1 / 3} \Gamma_{0}^{\prime} c k_{p}^{\prime}\right)$ for sevcral values of $\kappa_{M}$. The curves for $\kappa_{M}=0.1, \kappa_{M}=0.7$, and $\kappa_{M}=0.99$ are calculated from Eq.(141). The curve for $\kappa_{M}=1.0$ is calculated from Eq.(103). The same results are displayed in (a) a linear-scale plot, and (b) a log-scale plot. 


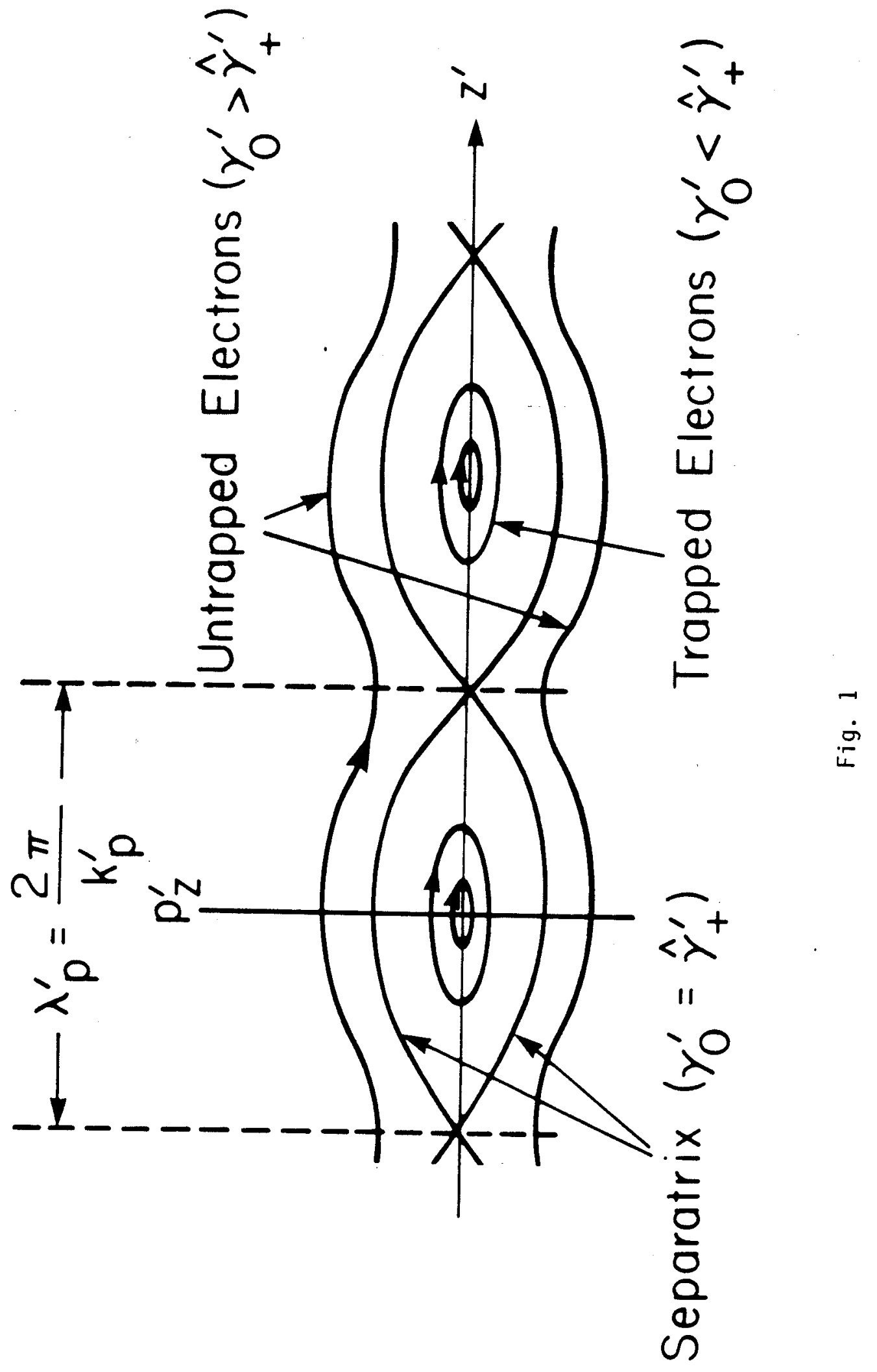




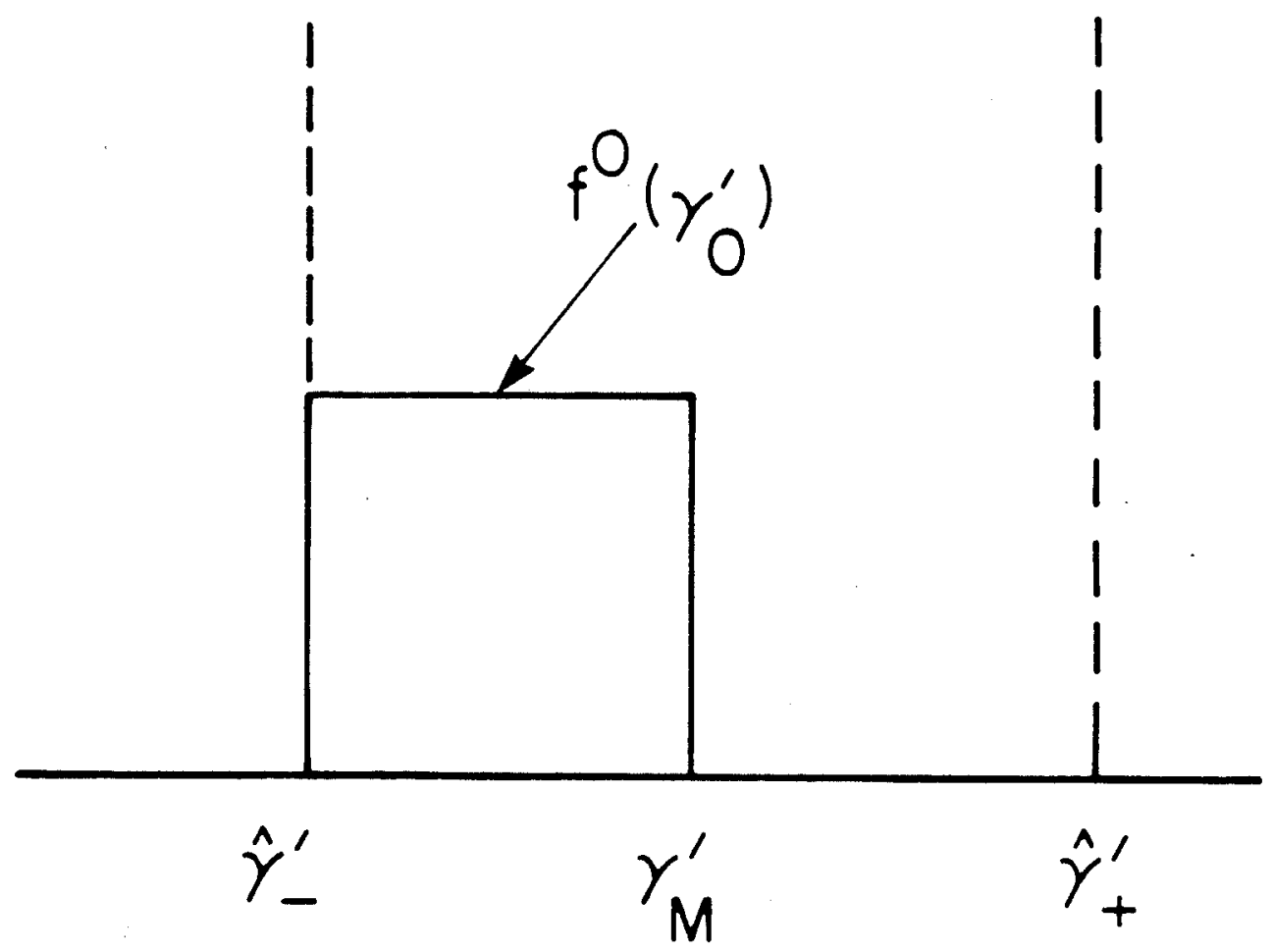

Fig. 2 

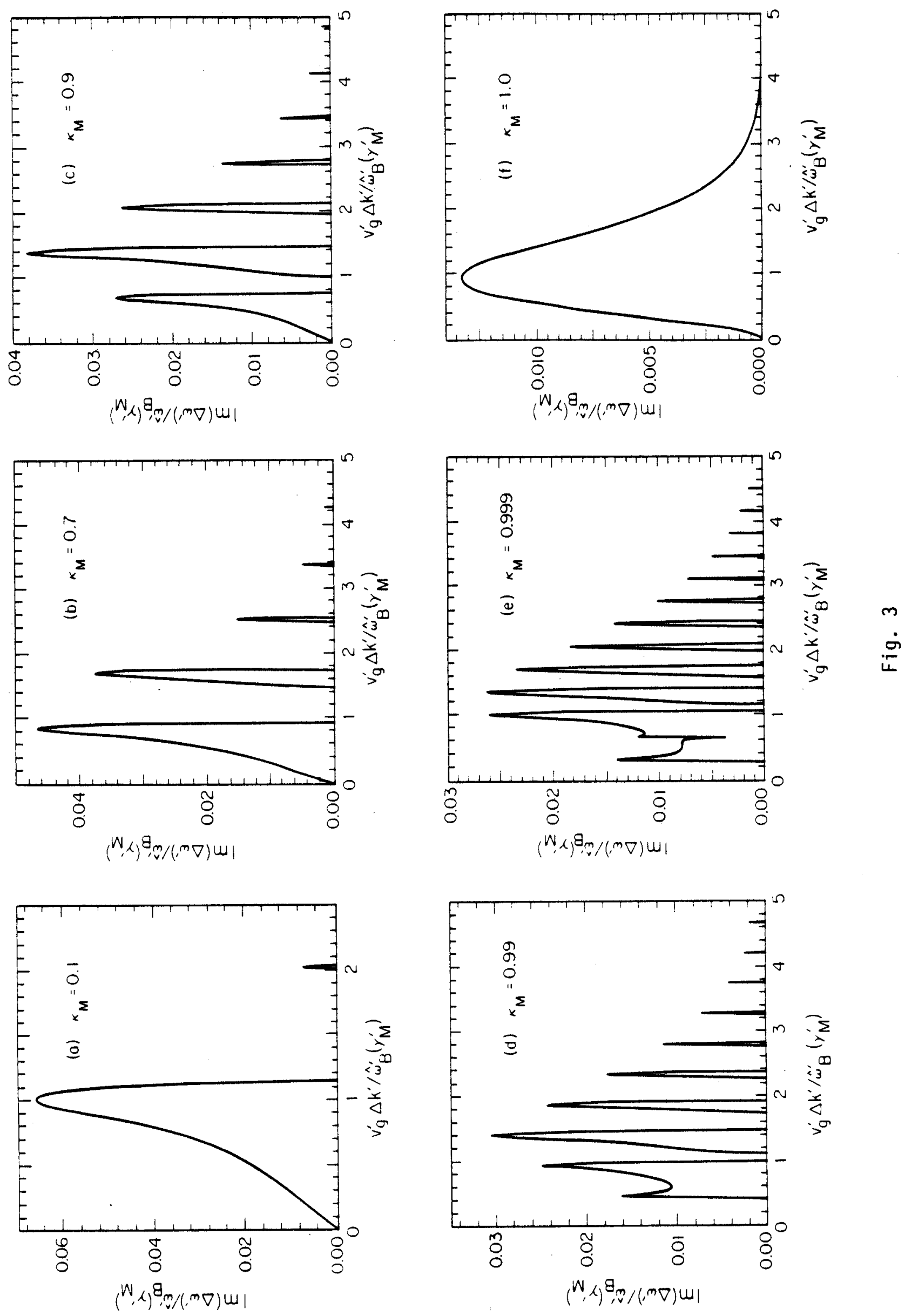

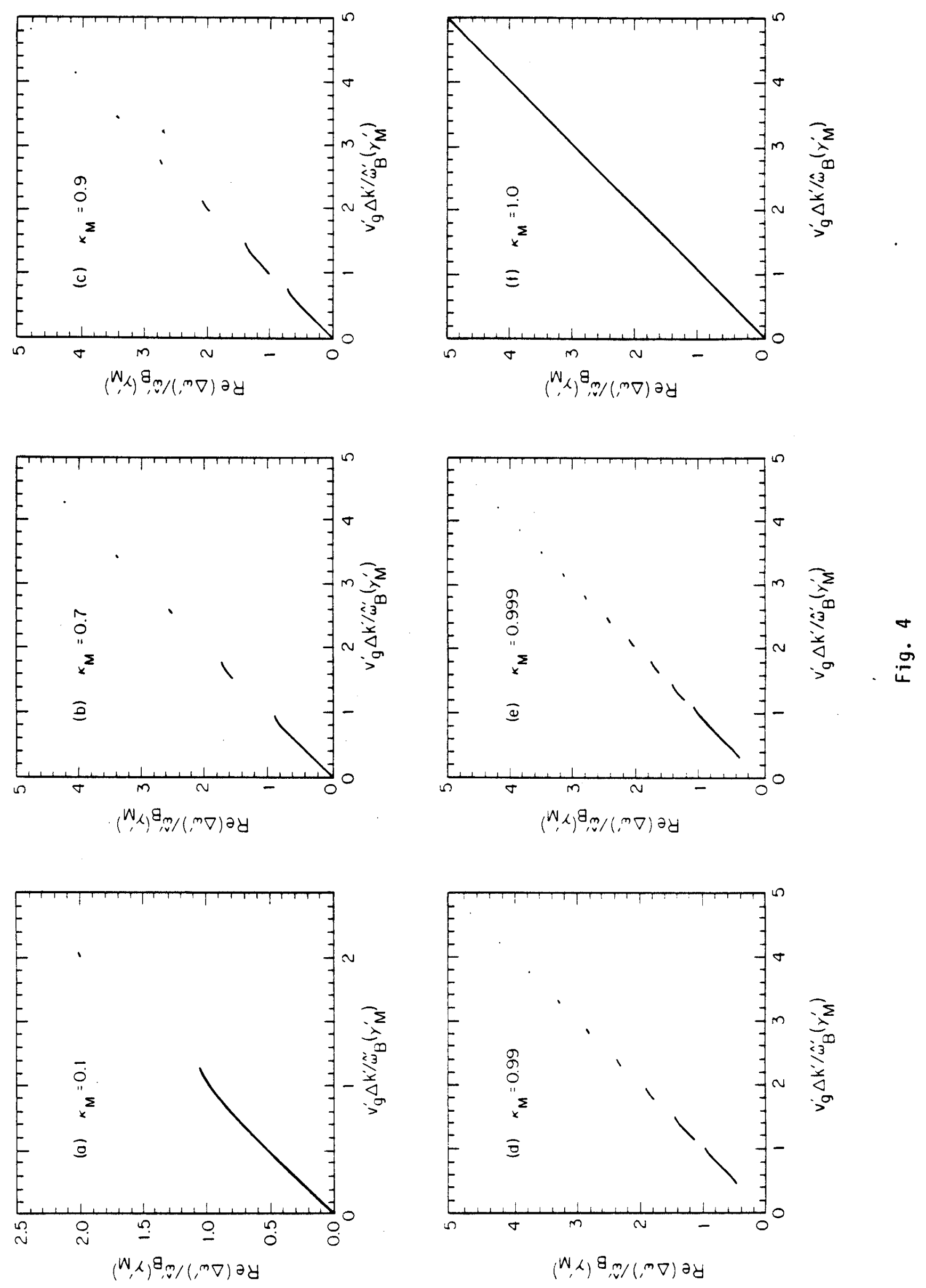

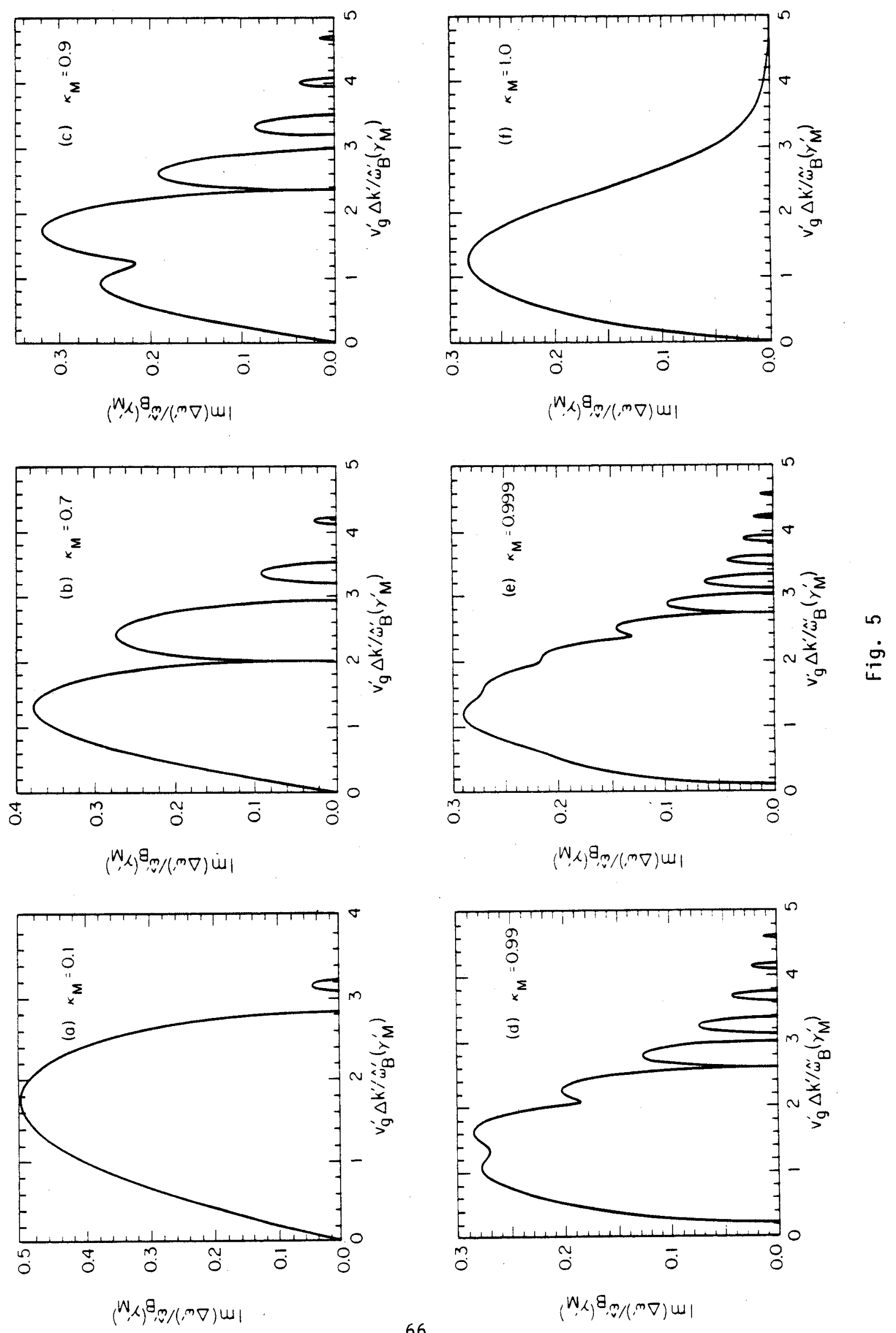

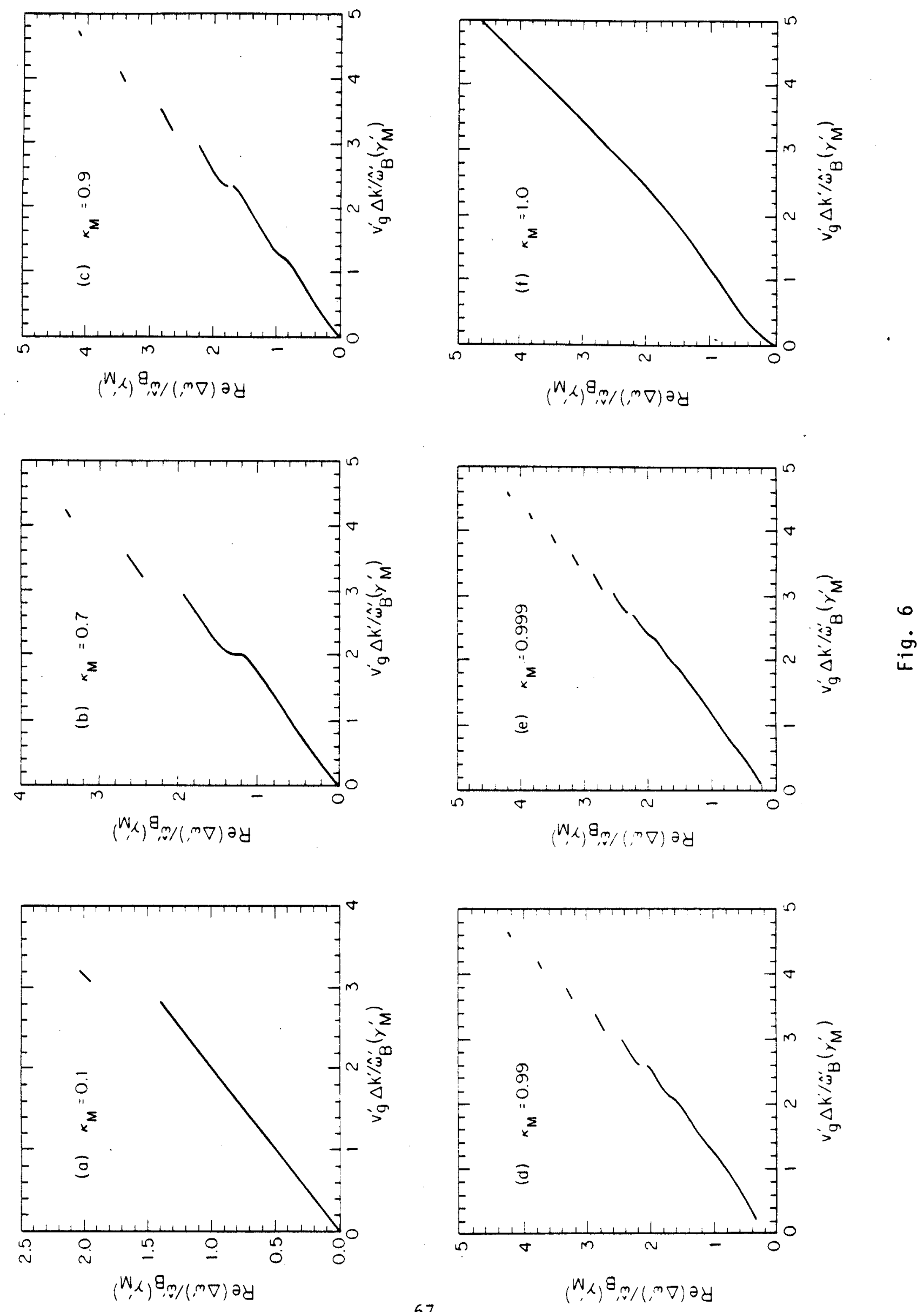

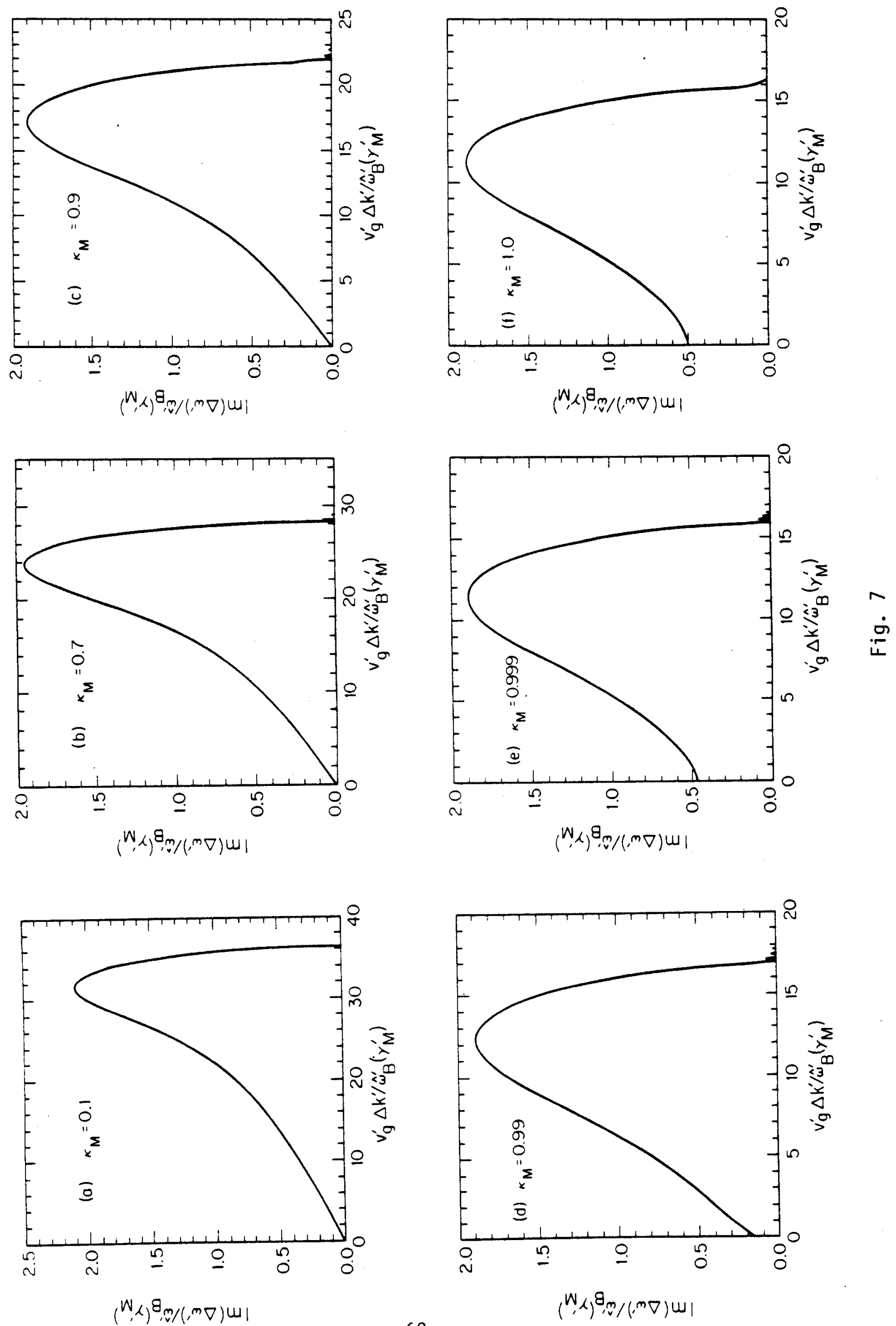


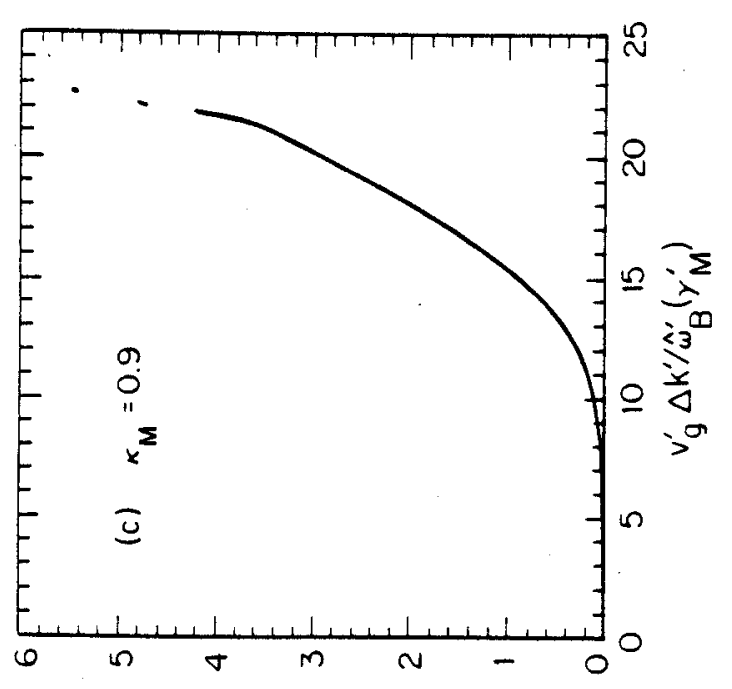

$\left.\left(w_{\mu}\right)_{m}, m /, m \nabla\right) \partial y$

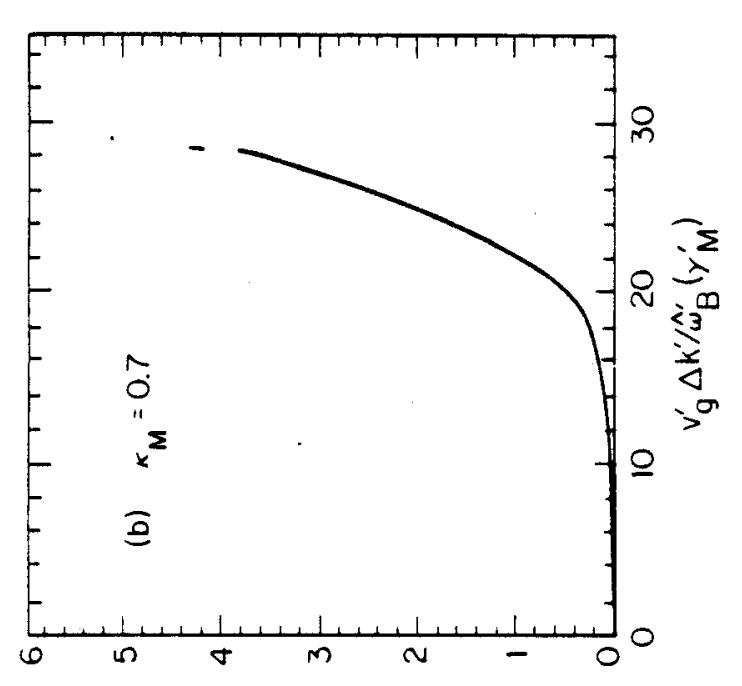

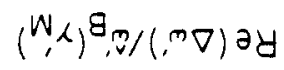

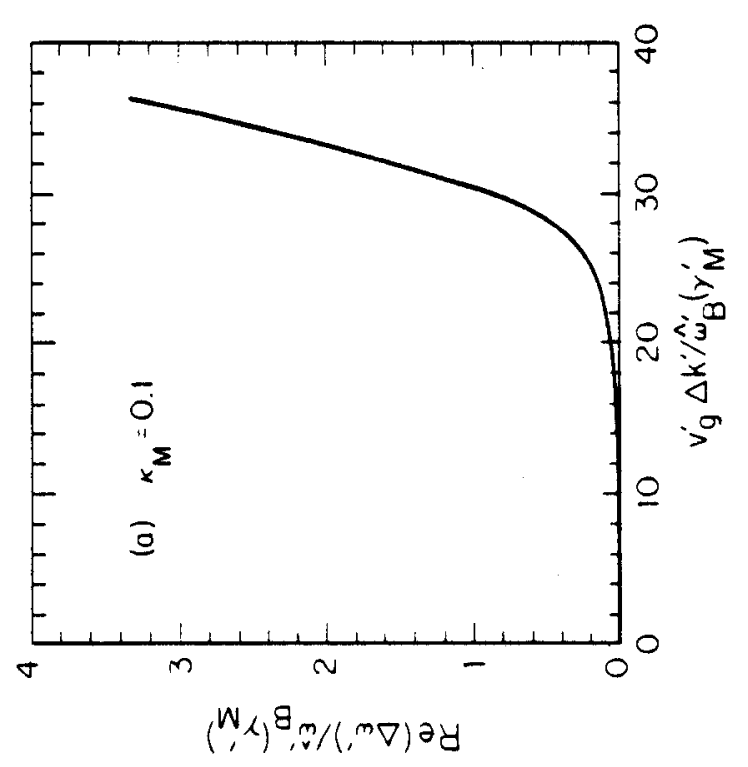

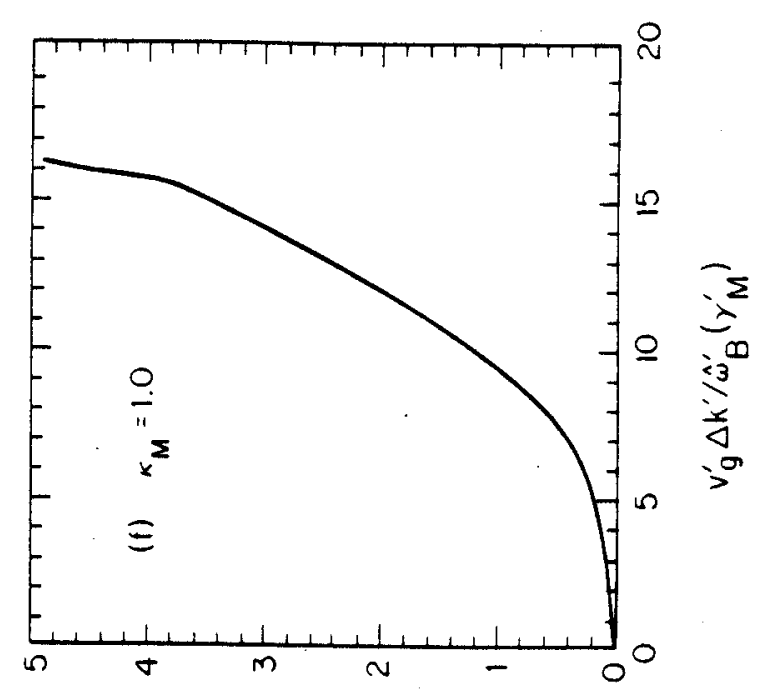

$\left(w_{l}, g_{, m /(m \nabla) \partial y}\right.$

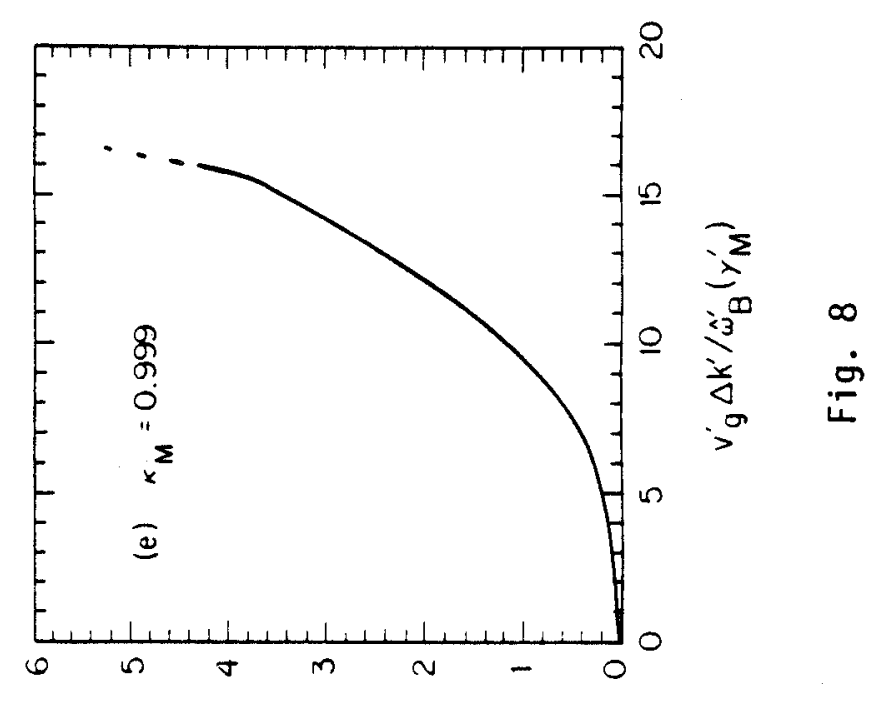

$\left(w, M \theta_{m /, m}, m\right) \partial y$

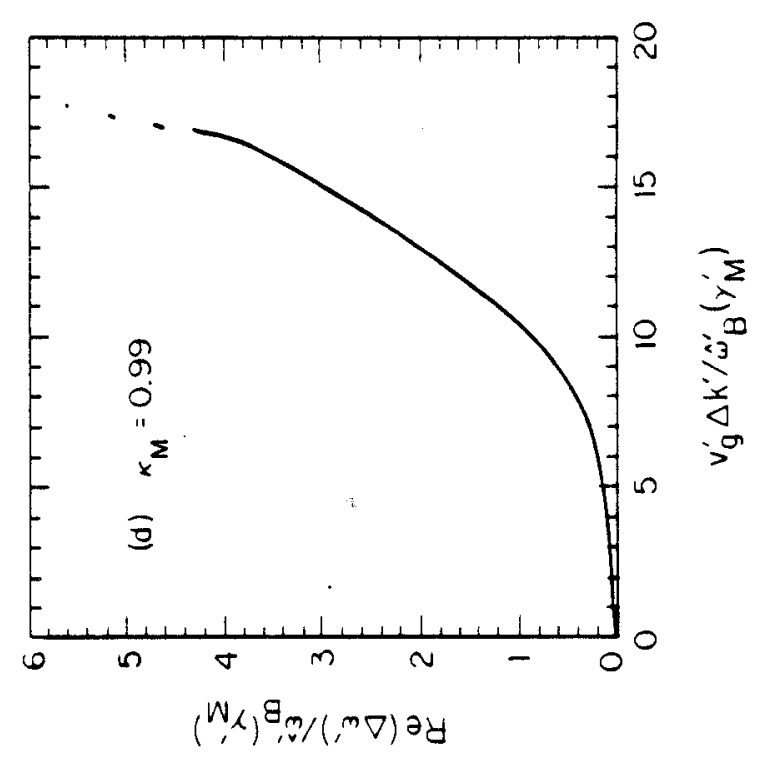




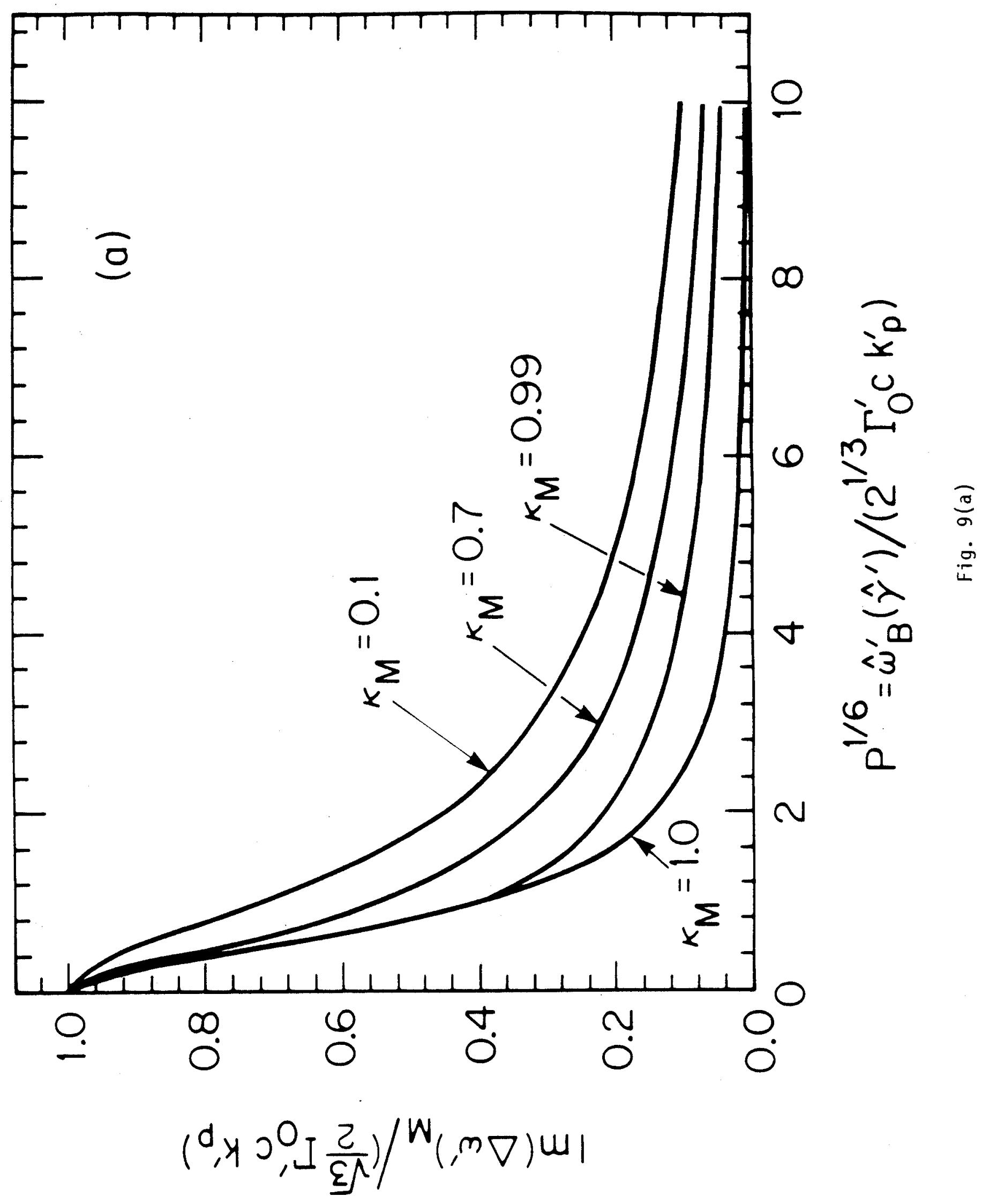




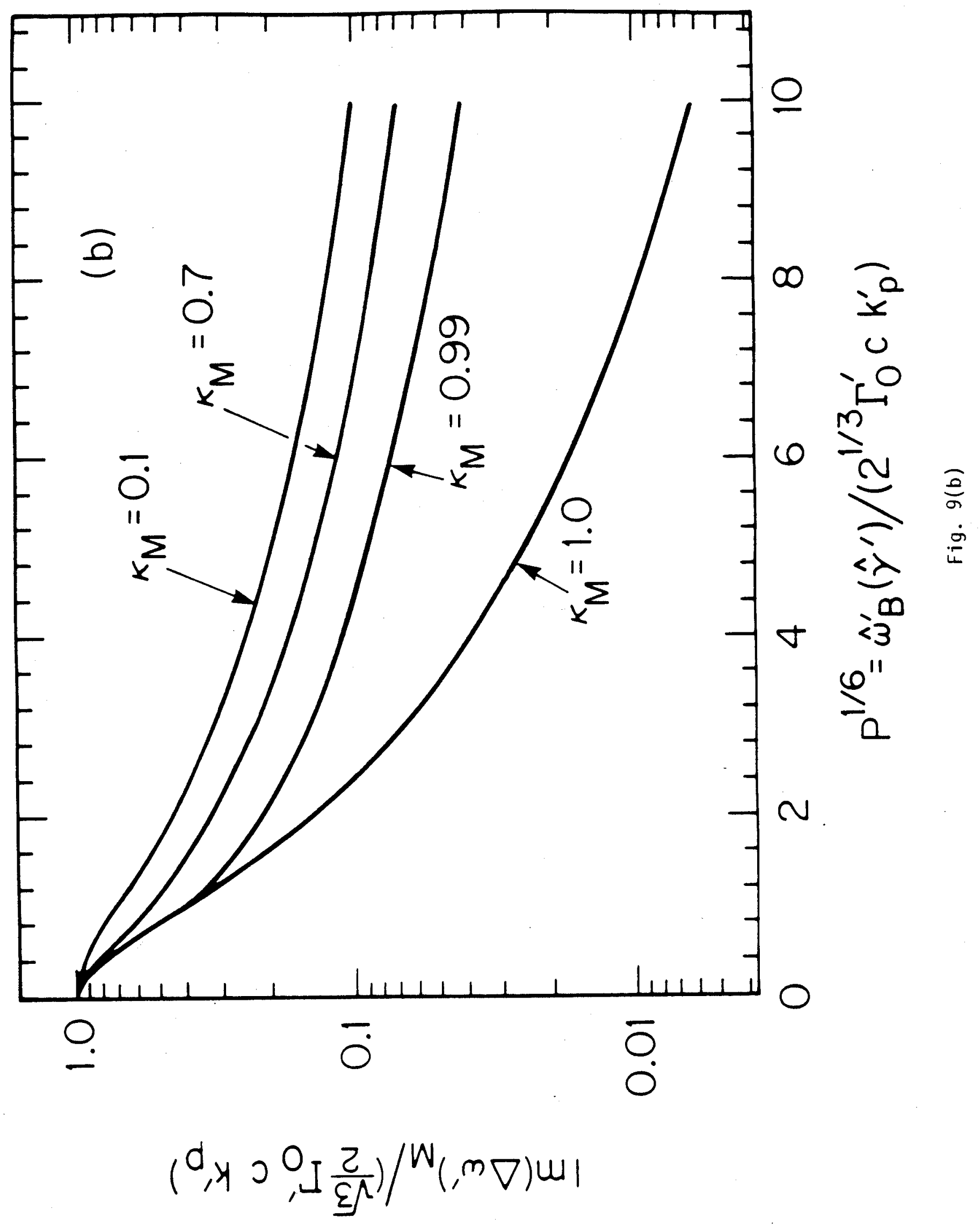

